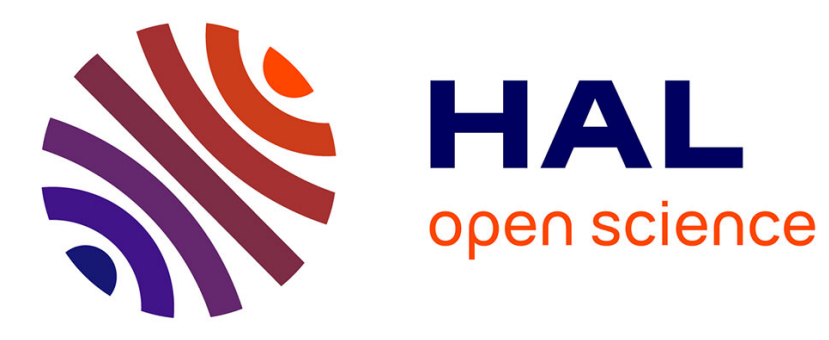

\title{
Sur la conjecture de Manin pour certaines surfaces de Châtelet
}

Régis de La Bretèche, Gérald Tenenbaum

\section{To cite this version:}

Régis de La Bretèche, Gérald Tenenbaum. Sur la conjecture de Manin pour certaines surfaces de Châtelet. Journal of the Institute of Mathematics of Jussieu, 2013, 12, pp.759-813. 10.1017/S1474748012000886 . hal-01281337

\section{HAL Id: hal-01281337 \\ https://hal.science/hal-01281337}

Submitted on 2 Mar 2016

HAL is a multi-disciplinary open access archive for the deposit and dissemination of scientific research documents, whether they are published or not. The documents may come from teaching and research institutions in France or abroad, or from public or private research centers.
L'archive ouverte pluridisciplinaire HAL, est destinée au dépôt et à la diffusion de documents scientifiques de niveau recherche, publiés ou non, émanant des établissements d'enseignement et de recherche français ou étrangers, des laboratoires publics ou privés. 


\title{
Sur la conjecture de Manin pour certaines surfaces de Châtelet
}

\author{
R. de la Bretèche* \& G. Tenenbaum
}

\begin{abstract}
We prove Manin's conjecture, in the strong form conjectured by Peyre, for Châtelet surfaces associated to surfaces of the type $y^{2}+z^{2}=P(x, 1)$, where $P$ is a binary quartic form with integer coefficients that is either irreducible over $\mathbb{Q}[i]$ or the product of two quadratic forms with integer coefficients and irreducible over $\mathbb{Q}[i]$. Moreover, we provide an explicit upper bound for the remainder term in the relevant asymptotic formula. This essentially settles Manin's conjecture for all Châtelet surfaces. The proof rests on two new tools, namely upper bounds for mean values of local oscillations of characters on divisors and sharp upper estimates for mean values of arithmetic functions of binary forms.
\end{abstract}

Keywords: Châtelet surfaces, Manin's conjecture, Peyre's constant, del Pezzo surfaces of degree 4 , count of lattice points over algebraic varieties, number of representations as a sum of two squares, method of descent.

Résumé. Nous démontrons, sous la forme forte conjecturée par Peyre, la conjecture de Manin pour les surfaces de Châtelet dont les équations sont du type $y^{2}+z^{2}=P(x, 1)$, où $P$ est une forme binaire quartique à coefficients entiers irréductible sur $\mathbb{Q}[i]$ ou produit de deux formes quadratiques à coefficients entiers irréductibles sur $\mathbb{Q}[i]$. De plus, nous fournissons une estimation explicite du terme d'erreur de la formule asymptotique sous-jacente. Cela finalise essentiellement la validation de la conjecture de Manin pour l'ensemble des surfaces de Châtelet. La preuve s'appuie sur deux méthodes nouvelles, concernant, du part, les estimations en moyenne d'oscillations locales de caractères sur les diviseurs, et, d'autre part, les majorations de certaines fonctions arithmétiques de formes binaires.

Mots clefs : Surfaces de Châtelet, conjecture de Manin, constante de Peyre, surfaces de del Pezzo de degré 4, comptage de points entiers sur des variétés algébriques, nombre de représentations comme somme de deux carrés, méthode de descente.

\section{Sommaire}

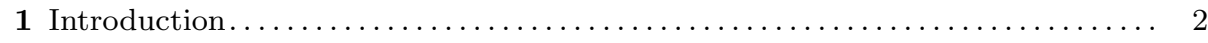

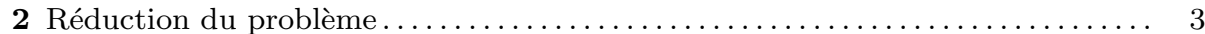

3 Sur le nombre de solutions locales d'une équation polynomiale........... 5

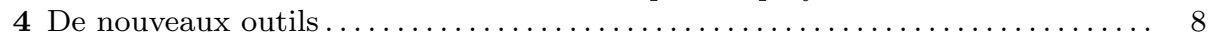

4.1 Moyennes de fonctions arithmétiques de formes binaires............. 8

4.2 Oscillations localisées de caractères sur les diviseurs............... 10

5 Géométrie des nombres.................................... 11

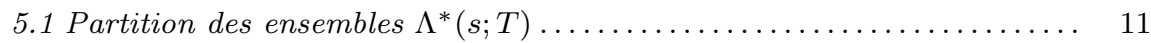

5.2 Répartition des points de $\Lambda(d ; T)$ dans les domaines convexes........ 12

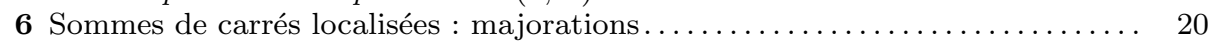

7 Sommes de carrés localisées : formules asymptotiques ................ 21

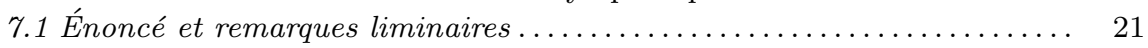

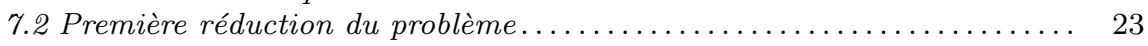

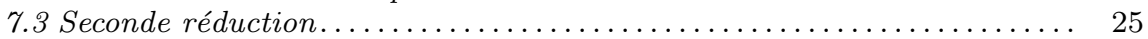

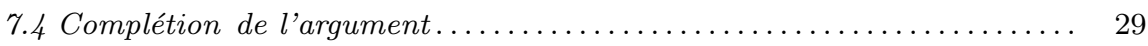

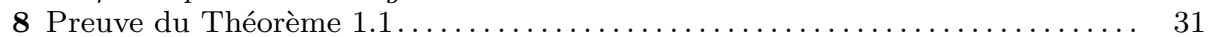

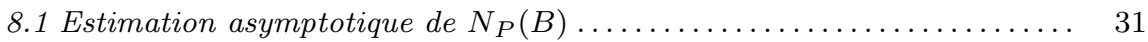

8.2 Validation de la conjecture de Peyre........................ 35

9 Le cas $P=P_{1} P_{2}:$ preuve du Théorème $1.2 \ldots \ldots \ldots \ldots \ldots \ldots \ldots \ldots \ldots \ldots \ldots$

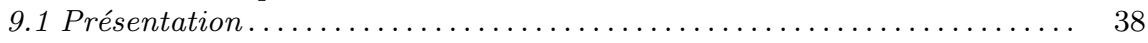

9.2 Sommes de carrés localisées : énoncé des formules asymptotiques..... 39

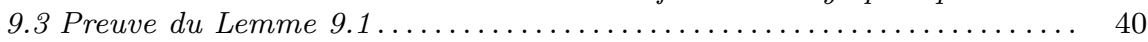

9.4 Estimation asymptotique de $N_{P}(B)$ lorsque $P=P_{1} P_{2} \ldots \ldots \ldots \ldots \ldots 46$

9.5 Validation de la conjecture de Peyre..................... 50 


\section{Introduction}

Soient $P \in \mathbb{Z}[X, Y]$ une forme binaire de degré 4 de discriminant non nul et $V$ un modèle propre et lisse de la surface affine

$$
y^{2}+z^{2}=P(x, 1)
$$

Alors $V$ est une surface de Châtelet sur $\mathbb{Q}$. La conjecture de Manin est relative à la répartition des points rationnels de cette variété. Elle propose un équivalent asymptotique pour le nombre $N_{P}(B)$ des points $\mathbb{Q}$-rationnels de $V$ dont la hauteur n'excède pas une borne déterminée $B$.

Il existe un morphisme $\psi: V \rightarrow \mathbb{P}^{4}$ dont l'image est une surface de del Pezzo de degré 4 . Désignant par $\|P\|$ la somme des modules des coefficients de $P$, nous nous donnons une hauteur $H$ sur $V$ définie par $H=H_{4} \circ \psi$, où $\left.H_{4}: \mathbb{P}^{4}(\mathbb{Q}) \rightarrow\right] 0, \infty[$ est une hauteur exponentielle associée à la norme

$$
\|\boldsymbol{x}\|_{P}=\max \left\{\left|x_{0}\right|,\left|x_{1}\right|,\left|x_{2}\right|, \frac{\left|x_{3}\right|}{\sqrt{\|P\|}}, \frac{\left|x_{4}\right|}{\sqrt{\|P\|}}\right\} \quad\left(\boldsymbol{x} \in \mathbb{R}^{5}\right) .
$$

Ces choix étant effectués, on peut montrer (voir le lemme 2 de [9]) que la quantité $N_{P}(B)$ à estimer dans le cadre de la conjecture de Manin vaut

$$
N_{P}(B):=\frac{1}{2} \sum_{\substack{(y, z, t, u, v) \in \mathbb{Z}^{5}, t>0 \\\left\|\left(v^{2} t, u v t, u^{2} t, y, z\right)\right\|_{P} \leqslant B \\ y^{2}+z^{2}=t^{2} P(u, v) \\(u, v)=(y, z, t)=1}} 1 .
$$

Lorsque les différents corps de rupture associés aux facteurs irréductibles de $P$ sur $\mathbb{Q}$ ne contiennent pas de racine de $X^{2}+1$, la conjecture de Manin s'énonce sous la forme

$$
N_{P}(B) \sim C_{H}(V) B \log B \quad(B \rightarrow \infty),
$$

où $C_{H}(V)$ est une constante géométrique dont la valeur, initialement calculée par Peyre [27], a été précisée au fur et à mesure des généralisations envisagées pour la variété $V$. Pour une présentation bibliographique plus complète du sujet, le lecteur pourra consulter en particulier [5] et [8].

Alors qu'une majoration générale par l'ordre de grandeur conjectural $N_{P}(B) \ll B \log B$ a été obtenue dans [9], l'examen de la forme précise $(1 \cdot 2)$ de la conjecture nécessite des approches distinctes en fonction des différentes factorisations possibles du polynôme $P$. Le cas où $P$ est scindé est traité dans [5]. Celui où $P$ possède un facteur cubique irréductible sur $\mathbb{Q}$ est résolu dans [4]. La situation où $P$ est le produit de deux formes linéaires et d'un facteur quadratique relève des méthodes de [3] et [5], dont une adaptation standard permet d'obtenir le résultat.

Nous proposons ici de traiter les deux cas résiduels, correspondant donc aux éventualités où $P$ est irréductible sur $\mathbb{Q}[i]$ et où $P$ est le produit de deux formes binaires de degré 2 à coefficients entiers, irréductibles sur $\mathbb{Q}[i]$ et non proportionnelles.

Nous obtenons les résultats suivants. Ici et dans toute la suite, nous notons $\|\cdot\|$ la norme euclidienne sur $\mathbb{R}^{2}$ et posons $\|x\|_{\infty}:=\max \left\{\left|x_{1}\right|,\left|x_{2}\right|\right\}$ pour $\boldsymbol{x}=\left(x_{1}, x_{2}\right) \in \mathbb{R}^{2}$.

Théorème 1.1. Soit $P$ une forme binaire de degré 4 de $\mathbb{Z}[X, Y]$, irréductible sur $\mathbb{Q}[i]$. On a

$$
N_{P}(B)=\left\{C_{H}(V)+O\left(\frac{1}{(\log B)^{1 / 100}}\right)\right\} B \log B \quad(B \rightarrow \infty),
$$

Dans les hypothèses de cet énoncé, la question de la positivité de $C_{H}(V)$ est théoriquement résolue de manière satisfaisante. En effet, il résulte du théorème $\mathrm{B}$ de [12] que le principe de Hasse s'applique dans ce cas. Ainsi, nous avons $C_{H}(V)>0$ si, et seulement si, $V$ possède au moins un point dans chaque complété de $\mathbb{Q}$. 
Théorème 1.2. Soit $P$ une forme binaire de degré 4 de $\mathbb{Z}[X, Y]$, qui est le produit de deux formes binaires de degré 2 à coefficients entiers, irréductibles sur $\mathbb{Q}[i]$ et non proportionnelles. On a

$$
N_{P}(B)=\left\{C_{H}(V)+O\left(\frac{1}{(\log B)^{1 / 70}}\right)\right\} B \log B \quad(B \rightarrow \infty) .
$$

La vérification du fait que la constante du terme principal est bien celle de Peyre cf. (9.43) infra — relève de la méthode développée dans [5]. Il est à noter que, dans le cas du Théorème 1.2 comme dans celui de toutes les surfaces de Châtelet, la seule obstruction possible au principe de Hasse est celle de Brauer-Manin — voir [10]. Le premier contre-exemple, correspondant au choix $P(X, Y)=\left(3 Y^{2}-X^{2}\right)\left(X^{2}-2 Y^{2}\right)$, a été exhibé dans [23]. L'étude générale de la validité du principe de Hasse pour l'ensemble des surfaces de Châtelet a été finalisée dans [12] et [13]. Nous vérifierons que $C_{H}(V)>0$ si, et seulement si, la surface $V$ contient un point rationnel.

Le Théorème 1.2 implique donc que, pour la classe de surfaces de Châtelet considérée, la seule obstruction au principe de Hasse est celle de Brauer-Manin. Cependant, il ne s'agit pas ici d'une nouvelle démonstration de ce principe puisque la paramétrisation des coordonnées des points rationnels repose sur les mêmes méthodes de descente que celles qui sont mises en œuvre dans [13].

L'extension de nos résultats aux surfaces du type $y^{2}-a z^{2}=P(1, x)$, où $a<0$ ne présente pas d'obstacle conceptuel. Lorsque le groupe des classes du corps $\mathbb{Q}(\sqrt{a})$ est trivial, nous pouvons écrire

$$
r_{a}(n):=\sum_{\substack{(s, t) \in \mathbb{Z}^{2} \\ s^{2}-a t^{2}=n}} 1=w_{a} \sum_{d \mid n} \chi_{a}(d)
$$

où $w_{a}$ désigne le nombre de racines de l'unité de $\mathbb{Q}(\sqrt{a})$ et $\chi_{a}$ est un caractère réel de module $4 a$ convenablement choisi. Lorsque le groupe des classes est non trivial, la situation est sensiblement plus délicate mais demeure abordable. En effet, la quantité $r_{a}(n)$ peut alors s'écrire comme une combinaison linéaire finie de fonctions analogues à celle du membre de droite de (1.5).

Cependant, en raison des complications techniques et méthodologiques liées aux places de mauvaise réduction et à l'occurrence des caractères de Hecke dans les extensions de (1.5), nous avons préféré ne pas inclure une telle généralisation dans ce travail : cela aurait impliqué une dilatation significative des démonstrations sans grand intérêt théorique. De même, nous n'avons pas considéré ici la question de l'approximation faible ${ }^{(1)}$ via l'équirépartition des points dans des voisinages adéliques.

Remerciements. Les auteurs prennent plaisir à exprimer ici leur gratitude envers, d'une part, Tim Browning, pour son aide lors de la phase d'élaboration, et, d'autre part, l'arbitre, dont la relecture attentive a permis d'améliorer significativement certains aspects de cette étude. Le premier auteur remercie également le Centre Interfacultaire Bernoulli de Lausanne pour son accueil au moment de la finalisation du présent travail.

\section{Réduction du problème}

Désignons par $r(n)$ le nombre de représentations d'un entier générique $n$ sous forme d'une somme de deux carrés et posons

$$
\begin{aligned}
\mathcal{R}_{P} & :=\left\{\boldsymbol{x} \in \mathbb{R}^{2}:\|\boldsymbol{x}\|_{\infty}<1, P(\boldsymbol{x})>0\right\}, \\
\mathcal{R}_{P}(\xi) & :=\left\{\boldsymbol{x} \in \mathbb{R}^{2}: \boldsymbol{x} / \xi \in \mathcal{R}_{P}\right\} \quad(\xi>0) .
\end{aligned}
$$

1. Voir notamment [10] et [11]. 
Des manipulations élémentaires permettent de réduire l'évaluation de $N_{P}(B)$ à celles de sommes de la forme

$$
\mathfrak{S}(\xi ; T):=\sum_{x \in \mathbb{Z}^{2} \cap \mathcal{R}_{T}(\xi)} r(T(\boldsymbol{x})), \quad \mathfrak{S}(\xi, d ; T):=\sum_{\substack{\boldsymbol{x} \in \mathbb{Z}^{2} \cap \mathcal{R}_{T}(\xi) \\ d \mid T(\boldsymbol{x})}} r\left(\frac{T(\boldsymbol{x})}{d}\right)
$$

où $T$ parcourt un ensemble de formes binaires construites à partir de $P$, sous réserve d'une uniformité adéquate relative aux coefficients de $T$.

Avec les notations

$$
\begin{aligned}
f_{d}(n) & :=\sum_{t k=n} \mu(k) r\left(d t^{2}\right) \quad\left(d, n \in \mathbb{N}^{*}\right), \\
S_{T}(\xi, d) & :=\sum_{\substack{(u, v) \in \mathbb{Z}^{2},(u, v)=1 \\
d|T(u, v)\\
| u|,| v \mid \leqslant \xi}} r\left(\frac{T(u, v)}{d}\right) \quad\left(\xi \geqslant 0, d \in \mathbb{N}^{*}\right),
\end{aligned}
$$

nous avons le résultat suivant.

Proposition 2.1. Soit $P \in \mathbb{Z}[X, Y]$ une forme binaire de degré 4 . Nous avons

$$
N_{P}(B)=\frac{1}{8} \sum_{d \leqslant B} \mu(d) \chi(d) \sum_{n \leqslant \min \left\{B / d, B d^{-3 / 2} \sqrt{\|P\|}\right\}} f_{d}(n) S_{P}\left(\sqrt{\frac{B}{d n}}, d\right) .
$$

Remarque. Seules les valeurs impaires de $d$ contribuent au membre de droite. Une inversion de Möbius fournit alors

$$
S_{P}(\xi, d)=\sum_{\ell \in \mathbb{N}^{*}} \mu(\ell) \mathfrak{S}\left(\xi / \ell, d_{\ell} ; P_{\ell}\right)
$$

où l'on a posé

$$
d_{\ell}:=\frac{d}{\left(d, \ell^{4}\right)}, \quad P_{\ell}(u, v):=\frac{\ell_{1}^{4} P(u, v)}{\left(d, \ell^{4}\right)}, \quad \ell_{1}:=\prod_{p^{\nu} \| \ell, p>2} p^{\nu} .
$$

L'introduction de la variable $\ell_{1}$ est ici justifiée par l'identité $r(2 n)=r(n)(n \geqslant 1)$ et par le fait que, lorsque $d$ est impair, $d \mid \ell^{4} P(u, v)$ équivaut à $d_{\ell} \mid P_{\ell}(u, v)$.

Démonstration. Posons

$$
\mathcal{D}:=\left\{d \in \mathbb{N}^{*}: p \mid d \Rightarrow p \equiv 1 \bmod 4\right\}, \quad r(n ; m)=\sum_{\substack{(a, b) \in \mathbb{Z}^{2} \\ n=a^{2}+b^{2} \\ \operatorname{pgcd}(m, a, b)=1}} 1 \quad\left(m, n \in \mathbb{N}^{*}\right)
$$

de sorte que l'on a identiquement $r(n ; 1)=r(n)$ et $r\left(m^{2} n ; m\right)=0$ lorsque $m \notin \mathcal{D}$.

En reportant dans (1.1) la formule d'inversion de Möbius

$$
r\left(y^{2} n ; y\right)=\sum_{k \mid y} \mu(k) r\left(\frac{y^{2} n}{k^{2}}\right)
$$


nous obtenons

$$
N_{P}(B)=\frac{1}{2} \sum_{k \leqslant B} \mu(k) \sum_{t \leqslant B / k} \sum_{\begin{array}{c}
(u, v) \in \mathbb{Z}^{2} \\
(u, v)=1 \\
|u|,|v| \leqslant \sqrt{B /(k t)}
\end{array}} r\left(t^{2} P(u, v)\right) .
$$

Soit $\chi$ le caractère de Dirichlet non principal de module 4. La relation ${ }^{(2)}$

$$
r(m n)=\frac{1}{4} \sum_{d \mid(m, n)} \mu(d) \chi(d) r\left(\frac{m}{d}\right) r\left(\frac{n}{d}\right)
$$

fournit alors

$$
\begin{aligned}
N_{P}(B) & =\frac{1}{8} \sum_{k \leqslant B} \mu(k) \sum_{d \leqslant B / k} \mu(d) \chi(d) \sum_{t \leqslant B /(k d)} r\left(d t^{2}\right) S_{P}\left(\sqrt{\frac{B}{k d t}}, d\right) \\
& =\frac{1}{8} \sum_{d \leqslant B} \mu(d) \chi(d) \sum_{n \leqslant B / d} S_{P}\left(\sqrt{\frac{B}{d n}}, d\right) f_{d}(n) .
\end{aligned}
$$

De plus, la condition de sommation $d \mid P(u, v)$ dans la somme intérieure implique $d \leqslant\|P\| B^{2} / d^{2} n^{2}$ et donc $n \leqslant B \sqrt{\|P\|} / d^{3 / 2}$.

Nous aurons l'usage d'évaluations concernant des sommes pondérées par la fonction $f_{d}$ lorsque $d \in \mathcal{D}$. Le lemme 14 de [4], énoncé ci-dessous, fournit l'estimation nécessaire.

Lemme 2.2. Nous avons uniformément pour $d \in \mathcal{D}, \mu(d)^{2}=1, \xi \geqslant 1$,

$$
\sum_{n \leqslant \xi} \frac{f_{d}(n)}{n}=\frac{r(d) \varphi^{\dagger}(d)}{\pi}\left\{\log \xi+O\left(\log ^{3}(\omega(d)+2)\right)\right\},
$$

où $\varphi^{\dagger}(d):=\prod_{p \mid d}(1+1 / p)^{-1}$.

Remarque. Le calcul de la constante dans $(2 \cdot 11)$ provient de l'identité

$$
\prod_{p}\left(1+\frac{\chi(p)}{p}\right)=\frac{2}{\pi}
$$

qui sera utilisée ultérieurement.

\section{Sur le nombre de solutions locales d'une équation polynomiale}

Ce paragraphe contient essentiellement des résultats classiques.

Commençons par introduire quelques notations valables pour toute la suite de l'article.

Nous désignons par $\mathcal{D}(T):=\operatorname{disc}(T)$ le discriminant d'un polynôme à une variable ou à deux variables, de sorte que $\mathcal{D}(T(X, Y))=\mathcal{D}(T(X, 1))=\mathcal{D}(T(1, Y))$.

Étant donnés $T \in \mathbb{Z}[X], s \in \mathbb{N}^{*}$, nous désignons par $\varrho_{T}(s)$ le nombre de racines de $T$ dans $\mathbb{Z} / s \mathbb{Z}$. Lorsque $T$ est une forme binaire de $\mathbb{Z}[X, Y]$, nous posons

$$
\varrho_{T}^{-}(s):=\sum_{\substack{1 \leqslant a \leqslant s \\ T(a, 1) \equiv 0(\bmod s)}} 1, \quad \varrho_{T}^{+}(s):=\sum_{\substack{1 \leqslant a, b \leqslant s \\ T(a, b) \equiv 0(\bmod s)}} 1 \quad(s \geqslant 1) .
$$

2. Cette formule classique est aisément vérifiable lorsque $m$ et $n$ sont des puissances de nombres premiers; le cas général s'en déduit par multiplicativité. 
Pour $T \in \mathbb{Z}[X, Y], s \in \mathbb{N}^{*}$, nous introduisons les ensembles

$$
\Lambda(s ; T):=\left\{(m, n) \in \mathbb{Z}^{2}: s \mid T(m, n)\right\}, \quad \Lambda^{*}(s ; T):=\{(m, n) \in \Lambda(s ; T):(m, n, s)=1\},
$$

de sorte que l'on a $\varrho_{T}^{+}(s):=\mid \Lambda(s ; T) \cap\left[0, s\left[^{2} \mid\right.\right.$ avec la notation introduite en $(3 \cdot 1)$. Nous posons également

$$
\varrho_{T}^{*}(s):=\mid \Lambda^{*}(s ; T) \cap\left[0, s\left[^{2} \mid \quad(s \geqslant 1) .\right.\right.
$$

De même, pour $\boldsymbol{T}=\left(T_{1}, T_{2}\right) \in \mathbb{Z}[X, Y], s=\left(s_{1}, s_{2}\right) \in \mathbb{N}^{* 2}$, nous posons

$$
\begin{aligned}
\Lambda\left(s_{1}, s_{2} ; T_{1}, T_{2}\right)=\Lambda(\boldsymbol{s} ; \boldsymbol{T}) & :=\left\{\boldsymbol{x} \in \mathbb{Z}^{2}: s_{1}\left|T_{1}(\boldsymbol{x}), s_{2}\right| T_{2}(\boldsymbol{x})\right\}, \\
\varrho_{\boldsymbol{T}}(\boldsymbol{s}) & :=\mid \Lambda(\boldsymbol{s} ; \boldsymbol{T}) \cap\left[0,\left.s_{1} s_{2}\right|^{2} \mid .\right.
\end{aligned}
$$

Ici et dans toute la suite de ce travail, la lettre minuscule $p$ désigne un nombre premier.

Lemme 3.1. Soit $T \in \mathbb{Z}[X]$ un polynôme primitif de degré $d$ et de discriminant $\mathcal{D}(T)$ non nul. Si $p^{\mu} \| \mathcal{D}(T)$, nous avons

$$
\varrho_{T}\left(p^{\nu}\right) \leqslant d p^{\min \{\nu-1,\lfloor(1-1 / d) \nu\rfloor, 2 \mu\}} .
$$

Démonstration. L'inégalité $\varrho_{T}\left(p^{\nu}\right) \leqslant d p^{\lfloor(1-1 / d) \nu\rfloor}$ est due à Stewart [30], les autres sont établies dans le livre de Nagell - cf. [26], théorèmes 52, 53 et 54, pp. 87-90.

L'énoncé suivant, qui est un cas particulier du lemme 2.2 de [7], permet de préciser les liens entre les quantités $\varrho_{T}^{-}(n)$ et $\varrho_{T}^{+}(n)$.

Lemme 3.2. Soit $T \in \mathbb{Z}[X, Y]$ une forme binaire primitive de degré 2 ou 4 et de discriminant $\mathcal{D}(T)$. Pour tout nombre premier $p \nmid T(1,0) \mathcal{D}(T)$ et tout entier $\nu \geqslant 1$, nous avons

$$
\varrho_{T}^{+}\left(p^{\nu}\right)= \begin{cases}p^{\nu} \varrho_{T}^{-}(p) \frac{p^{2\lceil\nu / 4\rceil}-1}{p^{2}(1+1 / p)}+p^{2(\nu-\lceil\nu / 4\rceil)} & \text { si } d=4, \\ p^{\nu} \varrho_{T}^{-}(p)\lceil\nu / 2\rceil(1-1 / p)+p^{2(\nu-\lceil\nu / 2\rceil)} & \text { si } d=2,\end{cases}
$$

et donc, en particulier, $\varrho_{T}^{+}(p)=(p-1) \varrho_{T}^{-}(p)+1, \varrho_{T}^{*}\left(p^{\nu}\right)=\varphi\left(p^{\nu}\right) \varrho_{T}^{-}\left(p^{\nu}\right)$.

De plus,

$$
\varrho_{T}^{+}\left(p^{\nu}\right) \leqslant(2 d+1) p^{\nu+\min \{\nu-1,\lfloor(d-1) \nu / d\rfloor\}} \quad(\nu \geqslant 1) .
$$

Nous nous restreignons ici au cas où $T \in \mathbb{Z}[X, Y]$ est irréductible, de degré $\geqslant 2$. On sait classiquement que la fonction zêta de Dedekind

$$
\zeta_{k}(s):=\sum_{\mathfrak{A}} \frac{1}{N_{k / \mathbb{Q}}(\mathfrak{A})^{s}} \quad(\Re e s>1)
$$

où $k$ est un corps de rupture du polynôme $T(X, 1)$ et $\mathfrak{A}$ décrit l'ensemble des idéaux entiers de $k$, reflète le comportement en moyenne de $\varrho_{T}^{-}(n)$. D'une part, $\zeta_{k}$ possède une décomposition en produit eulérien

$$
\zeta_{k}(s):=\prod_{\wp}\left(1-N_{k / \mathbb{Q}}(\wp)^{-s}\right)^{-1} \quad(\Re e s>1),
$$


où $\wp$ parcourt l'ensemble des idéaux premiers. D'autre part, pour tout nombre premier $p$ tel que $p \nmid \mathcal{D}(T)$, la factorisation

$$
T(X, 1)=\prod_{1 \leqslant j \leqslant r} T_{j}(X, 1)^{e_{j}}(\bmod p)
$$

où les $T_{j}$ sont des formes irréductibles de $\mathbb{Z} / p \mathbb{Z}[X]$ de degrés respectifs $f_{j}$, induit la décomposition

$$
p \mathbb{Z}=\prod_{1 \leqslant j \leqslant r} \wp_{j}^{e_{j}},
$$

avec $N_{k / \mathbb{Q}}\left(\wp_{j}\right)=p^{f_{j}}(1 \leqslant j \leqslant r)$. Ainsi $f_{j}=1$ signifie que $T_{j}$ possède une racine modulo $p$, et

$$
\varrho_{T}^{-}(p)=\left|\left\{\wp: N_{k / \mathbb{Q}}(\wp)=p\right\}\right| \quad(p \nmid \mathcal{D}(T)) .
$$

Lorsque $d=4$, nous pouvons donc déduire de ce qui précède et du Lemme 3.1 l'existence une fonction arithmétique multiplicative $h_{T}^{-}$telle que

$$
\sum_{n \geqslant 1} \frac{\varrho_{T}^{-}(n)}{n^{s}}=\zeta_{k}(s) \sum_{n \geqslant 1} \frac{h_{T}^{-}(n)}{n^{s}}
$$

et satisfaisant, pour tous $\kappa \in] 0, \frac{1}{4}[, \varepsilon>0$ fixés, à la majoration

$$
\sum_{n \geqslant 1} \frac{\left|h_{T}^{-}(n)\right|}{n^{1-\kappa}} \ll_{\kappa} \prod_{p \mid T(1,0) \mathcal{D}(f)}\left(1-p^{4 \kappa-1}\right)^{-16} \ll_{\varepsilon, \kappa}\|T\|^{\varepsilon} .
$$

Nous déduisons aisément des Lemmes 3.1, 3.2 et de (3.6) qu'il existe une fonction arithmétique multiplicative $h_{T}^{*}$ telle que

$$
\sum_{n \geqslant 1} \frac{\varrho_{T}^{*}(n)}{n^{s+1}}=\zeta_{k}(s) \sum_{n \geqslant 1} \frac{h_{T}^{*}(n)}{n^{s}} \quad(\Re e s>1)
$$

et satisfaisant, pour tout $\kappa \in] 0, \frac{1}{4}[$,

$$
\sum_{n \geqslant 1} \frac{\left|h_{T}^{*}(n)\right|}{n^{1-\kappa}} \ll_{\kappa} \prod_{p \mid T(1,0) \mathcal{D}(T)}\left(1-p^{4 \kappa-1}\right)^{O(1)} \ll\|T\|^{\varepsilon},
$$

pour tout $\varepsilon>0$ fixé.

De même, il existe une fonction $h_{T}^{+}$satisfaisant aux mêmes majorations et telle que

$$
\sum_{n \geqslant 1} \frac{\varrho_{T}^{+}(n)}{n^{s+1}}=\zeta_{k}(s) \sum_{n \geqslant 1} \frac{h_{T}^{+}(n)}{n^{s}} \quad(\Re e s>1) .
$$

Nous obtiendrons des renseignements sur le comportement en moyenne de $\chi(n) \varrho_{T}^{-}(n)$ grâce aux propriété analytiques de la série de Dirichlet

$$
L_{k}(s, \chi):=\sum_{\mathfrak{A}} \frac{\chi\left(N_{k / \mathbb{Q}}(\mathfrak{A})\right)}{N_{k / \mathbb{Q}}(\mathfrak{A})^{s}} \quad(\Re \text { e } s>1),
$$

où $k$ est le corps de rupture du polynôme $T(X, 1)$ et $\mathfrak{A}$ décrit l'ensemble des idéaux entiers de $k$. Des analogues des résultats mentionnés plus hauts pour $\zeta_{k}$ sont alors classiquement 
valables. Par exemple, il existe des fonctions $h_{T}^{+}(\cdot ; \chi)$ et $h_{T}^{-}(\cdot ; \chi)$ satisfaisant des majorations de type $(3 \cdot 9)$ et telles que

$$
\sum_{n \geqslant 1} \frac{\chi(n) \varrho_{T}^{ \pm}(n)}{n^{s+1}}=L_{k}(s, \chi) \sum_{n \geqslant 1} \frac{h_{T}^{ \pm}(n ; \chi)}{n^{s}} .
$$

Enfin, nous ferons appel au théorème des idéaux premiers et à sa version relative aux progressions arithmétiques sous la forme suivante. Comme il est l'usage, nous notons

$$
\operatorname{li}(x):=\int_{2}^{x} \frac{\mathrm{d} t}{\log t}
$$

le logarithme intégral.

Le résultat suivant est établi dans le survol d'Heilbronn [18].

Lemme 3.3. Soit $T$ une forme binaire irréductible sur $\mathbb{Q}[i]$. Il existe $c>0$ telle que l'on ait

$$
\begin{aligned}
& \sum_{p \leqslant x} \varrho_{T}^{-}(p)=\operatorname{li}(x)+O\left(x \mathrm{e}^{-c \sqrt{\log x}}\right) \\
& \sum_{p \leqslant x} \chi(p) \varrho_{T}^{-}(p) \ll x \mathrm{e}^{-c \sqrt{\log x}},
\end{aligned}
$$

où les constantes implicites dépendent au plus de la forme binaire $T$.

En particulier, la série $\sum_{p} \chi(p) \varrho_{T}^{-}(p) / p$ est convergente.

Remarque. L'estimation (3·12) est encore valable lorsque $T$ est irréductible sur $\mathbb{Q}$ mais pas sur $\mathbb{Q}[i]$.

\section{De nouveaux outils}

Les démonstrations des Théorèmes 1.1 et 1.2 reposent de manière essentielle sur des résultats nouveaux, développés à cette fin dans [6] et [7] et possédant un intérêt propre. Nous consacrons ce paragraphe à la description des théorèmes obtenus, en nous cantonnant au niveau de généralité directement utilisable dans le présent contexte.

\subsection{Moyennes de fonctions arithmétiques de formes binaires}

Étant donnés des paramètres $A_{1} \geqslant 1, A_{2} \geqslant 1, \varepsilon>0$, nous notons $\mathcal{M}\left(A_{1}, A_{2}, \varepsilon\right)$ la classe des fonctions arithmétiques $F$ positives ou nulles satisfaisant la condition

$$
F(a b) \leqslant \min \left\{A_{1}^{\Omega(a)}, A_{2} a^{\varepsilon}\right\} F(b)
$$

pour tout $a, b$ tels que $(a, b)=1$. Par ailleurs, nous rappelons la notation $\varrho_{T}^{+}$introduite en $(3 \cdot 1)$.

Théorème 4.1 ([7]). Soit $T \in \mathbb{Z}[X, Y]$ une forme binaire primitive de degré au plus 4 , irréductible sur $\mathbb{Q}$. Pour tous

$$
\delta \in] 0,1\left[, \quad A_{1} \geqslant 1, \quad A_{2} \geqslant 1,\right.
$$

il existe une constante $c_{0}$ telle que, uniformément sous les conditions

$$
0<\varepsilon \leqslant \delta / 4000, \quad F \in \mathcal{M}\left(A_{1}, A_{2}, \varepsilon\right), \quad \min (u, x) \geqslant c_{0} \max \{u, x,\|T\|\}^{\delta},
$$


on ait

$$
\sum_{m \leqslant u, n \leqslant x} F(|T(m, n)|) \ll u x E_{T}(u+x ; F)
$$

où la constante implicite dépend au plus de $A_{1}, A_{2}, \varepsilon, \delta$, et où l'on a posé

$$
E_{T}(v ; F):=\prod_{4<p \leqslant v}\left(1-\frac{\varrho_{T}^{+}(p)}{p^{2}}\right) \sum_{1 \leqslant s \leqslant v} F(s) \frac{\varrho_{T}^{+}(s)}{s^{2}} \quad(v \geqslant 1) .
$$

De plus, il existe une constante $C=C\left(A_{1}\right)>0$ telle que

$$
E_{T}(v ; F) \ll \prod_{p \mid D^{*}}\left(1+\frac{1}{p}\right)^{C} \exp \left(\sum_{\substack{p \leqslant v \\ p \nmid D^{*}}} \frac{\varrho_{T}^{-}(p)}{p}(F(p)-1)\right) \quad(v \geqslant 1),
$$

où l'on a posé $D^{*}:=\mathcal{D}(T)$.

Lorsque $\boldsymbol{T}=\left(T_{1}, T_{2}\right) \in \mathbb{Z}[X]^{2}$, nous définissons une fonction arithmétique de deux variables par

$$
\varrho_{T}^{+}(s):=\sum_{\substack{1 \leqslant \xi, \eta \leqslant s_{1} s_{2} \\ T_{h}(\xi, \eta) \equiv 0\left(\bmod s_{h}\right)}} 1 .
$$

Théorème $4.2([7])$. Soit $\boldsymbol{T}:=\left(T_{1}, T_{2}\right) \in \mathbb{Z}[X, Y]^{2}$ un vecteur dont les deux coordonnées sont des formes binaires primitives irréductibles sur $\mathbb{Q}$ de degré 2 non proportionnelles. Pour tous

$$
\delta \in] 0,1\left[, \quad A_{1} \geqslant 1, \quad A_{2} \geqslant 1,\right.
$$

il existe une constante $c_{0}$ telle que, uniformément sous les conditions

$$
0<\varepsilon \delta / 4000, \quad F_{1}, F_{2} \in \mathcal{M}\left(A_{1}, A_{2}, \varepsilon\right), \quad \min (u, x) \geqslant c_{0} \max \{u, x,\|Q\|\}^{\delta},
$$

on ait

$$
\sum_{m \leqslant u, n \leqslant x} F_{1}\left(\left|T_{1}(m, n)\right|\right) F_{2}\left(\left|T_{2}(m, n)\right|\right) \ll u x E_{\boldsymbol{T}}(u+x),
$$

où la constante implicite dépendent au plus de $A_{1}, A_{2}, \varepsilon, \delta$, et où l'on a posé

$$
E_{\boldsymbol{T}}\left(v ; F_{1}, F_{2}\right):=\prod_{4<p \leqslant v}\left(1-\frac{\varrho_{T_{1} T_{2}}^{+}(p)}{p^{2}}\right) \sum_{\substack{s \in \mathbb{N}^{* 2} \\ 1 \leqslant s_{1} s_{2} \leqslant v}} F_{1}\left(s_{1}\right) F_{2}\left(s_{2}\right) \frac{\varrho_{\boldsymbol{T}}^{+}(s)}{s_{1}^{2} s_{2}^{2}} .
$$

De plus, il existe $K=K\left(A_{1}\right)>0$ telle que, notant $D^{*}:=\mathcal{D}\left(T_{1} T_{2}\right)$, on ait

$$
E_{\boldsymbol{T}}\left(v ; F_{1}, F_{2}\right) \ll \prod_{p \mid D^{*}}\left(1+\frac{1}{p}\right)^{K} E_{T_{1}}\left(v ; F_{1}\right) E_{T_{2}}\left(v ; F_{2}\right) .
$$


Nous notons $M_{2}(\mathbb{Z})^{*}:=M_{2}(\mathbb{Z}) \cap G L_{2}(\mathbb{R})$ l'ensemble des matrices $2 \times 2$ à coefficients entiers et de déterminant non nul, muni d'une norme d'opérateur, notée $\|\cdot\|$. Le lemme technique suivant permettra de simplifier certaines démonstrations.

Lemme 4.3. Soient $J \in \mathbb{Z}[X, Y]$ une forme binaire irréductible sur $\mathbb{Q}$ de degré au plus 4, $A_{1} \geqslant 1, A_{2} \geqslant 1, \kappa>0, q \in \mathbb{N}^{*}, \varepsilon>0$, et $F \in \mathcal{M}\left(A_{1}, A_{2}, \varepsilon\right)$. On se donne en outre une matrice $M \in M_{2}(\mathbb{Z})^{*}$ et un entier $q \in \mathbb{N}^{*}$, et l'on suppose que la forme binaire

$$
T(\boldsymbol{x}):=J(M \boldsymbol{x}) / q \quad\left(\boldsymbol{x} \in \mathbb{R}^{2}\right)
$$

est à coefficients entiers. Il existe alors $\varepsilon_{1}>0$ tel que, pour tout $0<\varepsilon<\varepsilon_{1}$, et uniformément sous la condition

$$
v \geqslant\|M\|^{\kappa}+\mathrm{e}^{\kappa q}
$$

on ait

$$
E_{T}(v ; F) \ll\|T\|^{\varepsilon} E_{J}(v ; F), \quad E_{J}(v ; F) \ll \prod_{p \leqslant v}\left(1+\frac{\varrho_{J}^{+}(p)}{p}(F(p)-1)\right) .
$$

Démonstration. Ces majorations découlent de la validité de la relation $\varrho_{T}^{+}(n)=\varrho_{J}^{+}(n)$ sous l'hypothèse $(n, q$ dét $M)=1$. Nous omettons les détails.

\subsection{Oscillations localisées de caractères sur les diviseurs}

Étant donnée une fonction arithmétique $f$, nous posons

$$
\begin{aligned}
\Delta(n, f ; u, v) & :=\sum_{\substack{d \mid n \\
\mathrm{e}^{u}<d \leqslant \mathrm{e}^{u+v}}} f(d), \quad\left(n \in \mathbb{N}^{*}, u \in \mathbb{R}, v>0\right), \\
\Delta(n, f) & :=\sup _{u \in \mathbb{R}, 0 \leqslant v \leqslant 1}|\Delta(n, f ; u, v)| .
\end{aligned}
$$

Ainsi, les fonction $\Delta(n, f)$ sont des généralisations de la fonction $\Delta$ de Hooley, correspondant au cas $f=\mathbf{1}$ - cf. notamment [22], [17], [24] et les références indiquées dans ces travaux. Nous avons $n \mapsto \Delta(n, f) \in \mathcal{M}\left(A_{1}\right):=\cap_{\varepsilon>0} \cup_{A_{2}>0} \mathcal{M}\left(A_{1}, A_{2}, \varepsilon\right)$ pour tout $A_{1} \geqslant 2$ dès que, par exemple, $f$ est à valeurs dans le disque unité.

Désignons par $\mathcal{M}^{*}\left(A_{1}, c\right)$ la sous-classe de $\mathcal{M}\left(A_{1}\right)$ constituée des fonctions arithmétiques $g$ multiplicatives, vérifiant en outre les relations asymptotiques

$$
\begin{aligned}
& \sum_{p \leqslant x} g(p)=\operatorname{li}(x)+O\left(x \mathrm{e}^{-c \sqrt{\log x}}\right) \\
& \sum_{p \leqslant x} \chi(p) g(p) \ll x \mathrm{e}^{-c \sqrt{\log x}} \quad(x \rightarrow \infty) .
\end{aligned}
$$

La majoration suivante est un cas particulier du théorème principal de [6]. Nous utilisons la notation

$$
\mathcal{L}(x):=\exp \sqrt{\log _{2} x \log _{3} x} \quad(x \geqslant 16),
$$

où $\log _{k}$ désigne la $k$-ième itérée de la fonction logarithme. 
Théorème 4.4. Soient $A_{1}>0, c>0$, et $g \in \mathcal{N}^{*}\left(A_{1}, c\right)$. Il existe une constante $\alpha=\alpha(g)>0$ telle que l'on ait

$$
\sum_{n \leqslant x} g(n) \Delta(n, \chi)^{2} \ll x \mathcal{L}(x)^{\alpha} \quad(x \geqslant 16) .
$$

En particulier, pour toute forme binaire $T \in \mathbb{Z}[X, Y]$, irréductible sur $\mathbb{Q}[i]$, nous avons, avec la notation (4.4),

$$
E_{T}(x ; \Delta(\cdot, \chi)) \ll_{T} \mathcal{L}(x)^{\alpha_{T}} \quad(x \geqslant 16)
$$

avec $\alpha_{T}:=\alpha\left(\varrho_{T}^{+}\right)$.

En effet, une sommation d'Abel standard permet de déduire (4·11) de (4·10).

\section{Géométrie des nombres}

\subsection{Partition des ensembles $\Lambda^{*}(s ; T)$}

Nous effectuons ici quelques remarques préliminaires concernant les ensembles $\Lambda(s ; T)$.

Soient $T \in \mathbb{Z}[X, Y]$ une forme binaire et $s \in \mathbb{N}^{*}$. Dans $\Lambda^{*}(s ; T)$, nous définissons une relation d'équivalence en convenant que $\boldsymbol{x} \sim \boldsymbol{y}$ si et seulement s'il existe $\lambda \in \mathbb{Z}$ tel que

$$
\boldsymbol{y} \equiv \lambda \boldsymbol{x}(\bmod s),
$$

et nous notons que cela implique $(\lambda, s)=1$. L'ensemble $\mathcal{U}_{T}(s)$ des classes d'équivalence induit donc une partition de $\Lambda^{*}(s ; T)$. Pour tout $\mathcal{A} \in \mathcal{U}_{T}(s)$ et tout $\boldsymbol{x} \in \mathcal{A}$, nous avons

$$
\mathcal{A}=\left\{\boldsymbol{y} \in \Lambda^{*}(s ; T): \exists \lambda \in \mathbb{Z},(\lambda, s)=1, \boldsymbol{y} \equiv \lambda \boldsymbol{x}(\bmod s)\right\} .
$$

Comme $\lambda$ est déterminé modulo $s$ de manière unique par $\boldsymbol{y}$, nous avons $\mid \mathcal{A} \cap\left[0, s\left[^{2} \mid=\varphi(s)\right.\right.$, d'où

$$
\left|\mathcal{U}_{T}(s)\right|=\varrho_{T}^{*}(s) / \varphi(s) .
$$

De plus, pour toute classe $\mathcal{A}$ de $\mathcal{U}_{T}(s)$, tout diviseur $t$ de $s$, et tout $\boldsymbol{x}$ de $\mathcal{A}$, l'ensemble

$$
\mathcal{A}_{t}:=\left\{\boldsymbol{y} \in \mathbb{Z}^{2}: \exists \lambda \in \mathbb{Z}, \boldsymbol{y} \equiv \lambda \boldsymbol{x}(\bmod t)\right\} .
$$

est un sous-réseau de $\mathbb{Z}^{2}$ de rang 2 et de déterminant $t$, dont la définition ne dépend pas du choix de $\boldsymbol{x}$ dans $\mathcal{A}$.

Ici et dans la suite, nous définissons implicitement les coordonnées $m$ et $n$ d'un vecteur $\boldsymbol{x}$ de $\mathbb{Z}^{2}$ par la relation $\boldsymbol{x}=(m, n)$.

Lemme 5.1. Soit $T \in \mathbb{Z}[X, Y]$ une forme primitive. Nous avons uniformément pour $\xi \geqslant 0, s \in \mathbb{N}^{*}$,

$$
\sum_{\substack{\boldsymbol{x} \in \mathbb{Z}^{2},(m, n)=1 \\\|\boldsymbol{x}\| \leqslant \xi, s \mid T(\boldsymbol{x})}} 1 \ll \frac{\varrho_{T}^{*}(s)}{\varphi(s)}\left(\frac{\xi^{2}}{s}+1\right) .
$$

Démonstration. D'après ce qui précède, les vecteurs $\boldsymbol{x}$ à compter se répartissent dans des réseaux $\mathcal{A}_{s}$, de déterminant $s$, où $\mathcal{A}$ décrit $\mathcal{U}_{T}(s)$. D'après le lemme 3 de [18], on a pour toute classe $\mathcal{A}$ de $\mathcal{U}_{T}(s)$,

$$
\sum_{\substack{x \in A_{s},\|x\| \leqslant \xi \\(m, n)=1}} 1 \ll 1+\frac{\xi^{2}}{s} .
$$

La majoration annoncée résulte donc de $(5 \cdot 1)$. 


\subsection{Répartition des points de $\Lambda(d ; T)$ dans les domaines convexes}

Dans ce paragraphe, nous considérons sur une forme binaire $T \in \mathbb{Z}[X, Y]$ et un domaine $\mathcal{R} \subset \mathbb{R}^{2}$ satisfaisant aux hypothèses minimales suivantes :

$\left(H_{1}\right) \mathcal{R}$ est un ouvert borné dont la frontière est continûment différentiable par morceaux;

$\left(H_{2}\right) T$ est irréductible sur $\mathbb{Q}[i]$, de degré 4 ,

$\left(H_{3}\right) T(\boldsymbol{x})>0$ pour tout $\boldsymbol{x} \in \mathcal{R}$.

En cohérence avec $(2 \cdot 2)$, nous posons

$$
\mathcal{R}(\xi):=\left\{\boldsymbol{x} \in \mathbb{R}^{2}: \boldsymbol{x} / \xi \in \mathcal{R}\right\} .
$$

Les quantités suivantes constituent des mesures du diamètre de $\mathcal{R}$ relativement à distance euclidienne et à la distance associée à $T$.

$$
\delta_{\infty}=\delta_{\infty}(\mathcal{R}):=\sup _{\boldsymbol{x} \in \mathcal{R}}\|\boldsymbol{x}\|, \quad \delta_{T}=\delta_{T}(\mathcal{R}):=\sup _{\boldsymbol{x} \in \mathcal{R}}|T(\boldsymbol{x})|^{1 / 4} .
$$

Des raisons techniques nous amèneront à restreindre les sommations de type $(2 \cdot 3)$ aux vecteurs $\boldsymbol{x}$ de $\mathbb{Z}^{2}$ tels que $m \equiv 1 \bmod 2:$ cela revient à y remplacer le domaine $\mathcal{R} \cap \mathbb{Z}^{2}$ par

$$
\mathcal{R}^{\dagger}:=\left\{\boldsymbol{x} \in \mathbb{Z}^{2} \cap \mathcal{R}: m \equiv 1(\bmod 2)\right\} .
$$

Nous nous proposons alors de majorer

$$
\Phi=\Phi(\xi, y ; T, \sigma, \vartheta):=\sum_{\substack{1 \leqslant d \leqslant y \\ 2 \nmid d}} \sup _{\mathcal{R}}|| \Lambda(d ; T) \cap \mathcal{R}^{\dagger}(\xi)\left|-\operatorname{vol}(\mathcal{R}) \xi^{2} \frac{\varrho_{T}^{+}(d)}{2 d^{2}}\right|,
$$

où le supremum est pris sur l'ensemble des parties convexes $\mathcal{R}$ de $\mathbb{R}^{2}$ telles que $\delta_{\infty}(\mathcal{R}) \leqslant \sigma$, $\delta_{T}(\mathcal{R}) \leqslant \vartheta$, et dont la longueur de la frontière est $\ll \sigma$. Notons que $\delta_{T}(\mathcal{R}) \leqslant\|T\|^{1 / 4} \delta_{\infty}(\mathcal{R})$, de sorte que nous pouvons supposer sans perte de généralité que $\vartheta \leqslant\|T\|^{1 / 4} \sigma$.

Nous rappelons la définition de la fonction $\mathcal{L}$ en $(4 \cdot 9)$.

Lemme 5.2. Soient $\varepsilon>0, \kappa>0, J \in \mathbb{Z}[X, Y]$ une forme binaire irréductible sur $\mathbb{Q}$ de degré $4, M \in M_{2}(\mathbb{Z})^{*}, q \in \mathbb{N}^{*}$, et $\mathcal{R}$ un domaine de $\mathbb{R}^{2}$ vérifiant $\left(H_{1}\right)$. Sous l'hypothèse que la forme binaire $T$ définie par $T(\boldsymbol{x})=J(M \boldsymbol{x}) / q\left(\boldsymbol{x} \in \mathbb{R}^{2}\right)$ est à coefficients entiers et vérifie $\left(H_{2}\right)$ et $\left(H_{3}\right)$, et sous les conditions

$$
y \geqslant 2, \quad \xi \geqslant \mathrm{e}^{\kappa q}+\|M\|^{\kappa}, \quad 1 / \sqrt{\xi} \leqslant \sigma \leqslant \xi^{3 / 2}, \quad 1 / \sqrt{\xi} \leqslant \vartheta \leqslant \xi^{3 / 2}, \quad \vartheta \leqslant\|T\|^{1 / 4} \sigma,
$$

nous avons

$$
\Phi(\xi, y ; T, \sigma, \vartheta) \ll\|T\|^{\varepsilon}((\sigma+\vartheta) \xi \sqrt{y}+y) \mathcal{L}(\sigma \xi)^{\sqrt{2}+\varepsilon} .
$$

De plus,

$$
\Phi(\xi, y ; T, \sigma, \vartheta) \ll\|T\|^{\varepsilon}\left(\sigma^{2}+\vartheta^{2}\right) \xi^{2} \log _{2} \xi .
$$

Les constantes implicites de ces deux majorations dépendent au plus de $\kappa, \varepsilon, J$.

Remarques.

(i) Roger Heath-Brown, que nous prenons plaisir à remercier ici, nous a indiqué comment remplacer, dans le membre de droite de (5.5), le facteur $\mathcal{L}(\sigma \xi)^{\sqrt{2}+\varepsilon}$ par $\|T\|^{1 / 2}\left(\log _{2} \xi\right)^{2}$. Cette précision peut être utile dans certaines circonstances. Nous indiquons brièvement, immédiatement après la démonstration qui suit, les modifications nécessaires pour établir cette variante. Il est toutefois à noter qu'une dépendance aussi faible que possible en $\|T\|$ est indispensable pour atteindre les objectifs principaux du présent travail. 
(ii) Un résultat comparable aux majorations $(5 \cdot 5)$ et $(5 \cdot 6)$ a été établi par Daniel au lemme 3.3 de [15]. Nous précisons ici l'uniformité en $T$ et affranchissons le majorant de toute puissance de $\log (\sigma \xi)$. Ce gain, essentiel, permet d'obtenir, pour toute constante $c>0$, une estimation non triviale dès que $y \leqslant \sigma^{2} \xi^{2} /(\log \xi)^{c}$ - la majoration (5.6) étant plus précise que (5.5) lorsque $y$ est grand devant $\sigma^{2} \xi^{2}$.

(iii) Les conditions $1 / \sqrt{\xi} \leqslant \sigma \leqslant \xi^{3 / 2}, 1 / \sqrt{\xi} \leqslant \vartheta \leqslant \xi^{3 / 2}$ garantissent que

$$
\log (\sigma \xi) \asymp \log (\vartheta \xi) \asymp \log \xi
$$

(iv) Une majoration triviale s'écrit

$$
\Phi(\xi, y ; T, \sigma, \vartheta) \ll \sum_{x \in \mathcal{R}(\xi)} \tau(T(\boldsymbol{x}))+\sigma^{2} \xi^{2} \sum_{\substack{1 \leqslant d \leqslant y \\ 2 \nmid d}} \frac{\varrho_{T}^{+}(d)}{2 d^{2}} \ll\|T\|^{\varepsilon} \sigma^{2} \xi^{2} \log (\xi y),
$$

où $\tau$ désigne la fonction nombre de diviseurs et où nous avons utilisé le Théorème 4.1, puis le Lemme 4.3, pour obtenir

$$
E_{T}(\sigma \xi ; \tau) \ll\|T\|^{\varepsilon} \log \xi
$$

Démonstration. La majoration (5.5) découlant directement de (5.7) lorsque $y \geqslant \sigma^{2} \xi^{2} \log \xi$, nous pouvons supposer dans la suite que $y \leqslant \sigma^{2} \xi^{2} \log \xi$.

Nous pouvons aussi, classiquement (voir par exemple lemme 5 de [3]), nous restreindre au cas où la forme $T$ est primitive. On alors

$$
\Lambda(d ; T)=\bigsqcup_{b \mid \psi(d)} b \Lambda^{*}\left(d_{b} ; T\right)
$$

avec $d_{b}:=d /\left(d, b^{4}\right)$, et $\psi(d):=\prod_{p^{\nu} \| d} p^{\lceil\nu / 4\rceil}$.

Adoptant l'approche de [15], nous commençons par établir les analogues de (5.5) et (5.6) pour $\Lambda^{*}(s ; T)$. Il s'agit donc d'estimer

$$
\Phi^{*}=\Phi^{*}(\xi, y ; T, \sigma, \vartheta):=\sum_{\substack{1 \leqslant s \leqslant y \\ 2 \nmid s}} \sup _{\mathcal{R}}|| \Lambda^{*}(s ; T) \cap \mathcal{R}^{\dagger}(\xi)\left|-\frac{\varrho_{T}^{*}(s) \operatorname{vol}(\mathcal{R}) \xi^{2}}{2 s^{2}}\right|
$$

Lorsque $2 \nmid s$, on a

$$
\begin{aligned}
|| \Lambda^{*}(s ; T) \cap \mathcal{R}^{\dagger}(\xi) \mid- & \frac{\varrho_{T}^{*}(s) \operatorname{vol}(\mathcal{R}) \xi^{2}}{2 s^{2}}|=| \sum_{\mathcal{A} \in \mathcal{U}_{T}(s)}\left(\left|\mathcal{A} \cap \mathcal{R}^{\dagger}(\xi)\right|-\frac{\varrho_{T}^{*}(s) \operatorname{vol}(\mathcal{R}) \xi^{2}}{2\left|\mathcal{U}_{T}(s)\right| s^{2}}\right) \mid \\
& \leqslant \sum_{\mathcal{A} \in \mathcal{U}_{T}(s)}|| \mathcal{A} \cap \mathcal{R}^{\dagger}(\xi)\left|-\frac{\varphi(s) \operatorname{vol}(\mathcal{R}) \xi^{2}}{2 s^{2}}\right| .
\end{aligned}
$$

Comme $\mathcal{A}=\left\{(m, n) \in \mathcal{A}_{s}:(m, n, s)=1\right\}$, la formule d'inversion de Möbius permet d'écrire, lorsque $2 \nmid s$,

$$
\left|\mathcal{A} \cap \mathcal{R}^{\dagger}(\xi)\right|=\sum_{x \in \mathcal{A}_{s} \cap \mathcal{R}^{\dagger}(\xi)} \sum_{\substack{b \mid(m, n, s) \\ 2 \nmid b}} \mu(b)=\sum_{b \mid s, 2 \nmid b} \mu(b) \sum_{\substack{x \in \mathcal{R}^{\dagger}(\xi / b) \\ b x \in \mathcal{A}_{s}}} 1 \quad\left(\mathcal{A} \in \mathcal{U}_{T}(s)\right) .
$$


Pour chaque diviseur $b$ de $s$, la condition $b \boldsymbol{x} \in \mathcal{A}_{s}$ équivaut à l'existence de $(\lambda, \boldsymbol{y}) \in \mathbb{Z} \times \mathcal{A}$ tel que $b \boldsymbol{x} \equiv \lambda \boldsymbol{y}(\bmod s)$. Puisque $\left(y_{1}, y_{2}, s\right)=1$, on a nécessairement $b \mid \lambda$. Autrement dit, la condition $b \boldsymbol{x} \in \mathcal{A}_{s}$ équivaut à l'existence de $(\nu, \boldsymbol{y}) \in \mathbb{Z} \times \mathcal{A}$ tel que $\boldsymbol{x} \equiv \nu \boldsymbol{y}(\bmod s / b)$, et nous obtenons

$$
\left|\mathcal{A} \cap \mathcal{R}^{\dagger}(\xi)\right|=\sum_{b \mid s, 2 \nmid b} \mu(b)\left|\mathcal{R}^{\dagger}(\xi / b) \cap \mathcal{A}_{s / b}\right| \quad\left(\mathcal{A} \in \mathcal{U}_{T}(s)\right) .
$$

Posons $t:=s / b$. On sait classiquement (voir par exemple le lemme 2.1 de [15]) qu'il existe une base minimale $\left(\boldsymbol{v}_{t}, \boldsymbol{w}_{t}\right)=\left(\boldsymbol{v}_{t}(\mathcal{A}), \boldsymbol{w}_{t}(\mathcal{A})\right)$ de $\mathcal{A}_{t}$ dont les vecteurs satisfont

$$
t \leqslant\left\|\boldsymbol{v}_{t}\right\|\left\|\boldsymbol{w}_{t}\right\| \leqslant \sqrt{2} t, 0<\left\|\boldsymbol{v}_{t}\right\| \leqslant \sqrt{2 t},\left\|\boldsymbol{v}_{t}\right\| \leqslant\left\|\boldsymbol{w}_{t}\right\|,\left|\left\langle\boldsymbol{v}_{t}, \boldsymbol{w}_{t}\right\rangle\right| \leqslant \frac{1}{2}\left\|\boldsymbol{v}_{t}\right\|\left\|\boldsymbol{w}_{t}\right\|,
$$

où $\|\cdot\|$ désigne la norme euclidienne et $\langle\cdot, \cdot\rangle$ le produit scalaire associé. De plus, il découle immédiatement de la dernière inégalité que l'on a

$$
\left(\forall(\lambda, \nu, z) \in \mathbb{Z}^{2} \times \mathbb{R}^{+}\right) \quad\left\|\lambda \boldsymbol{v}_{t}+\nu \boldsymbol{w}_{t}\right\| \leqslant z \Rightarrow|\lambda| \leqslant \frac{2 z}{\left\|\boldsymbol{v}_{t}\right\|},|\nu| \leqslant \frac{2 z}{\left\|\boldsymbol{w}_{t}\right\|} .
$$

Comme l'abscisse d'un vecteur de $\mathcal{R}^{\dagger}$ est un entier impair, donc non nul, nous déduisons de ce qui précède que $\mathcal{R}^{\dagger}(\xi / b) \cap \mathcal{A}_{t}=\varnothing$ dès que $\left\|\boldsymbol{v}_{t}\right\|>2 \sigma \xi / b$. D'où, en vertu de l'approximation classique du nombre de points entiers dans un domaine convexe avec terme d'erreur majoré linéairement en fonction du périmètre,

$$
\begin{aligned}
\left|\mathcal{R}^{\dagger}(\xi / b) \cap \mathcal{A}_{t}\right| & =\frac{\operatorname{vol}(\mathcal{R}(\xi / b))}{2 t}+O\left(\frac{\sigma \xi}{b\left\|\boldsymbol{v}_{t}\right\|}+\min \left\{1, \frac{\sigma^{2} \xi^{2}}{b s}\right\}\right) \\
& =\frac{\operatorname{vol}(\mathcal{R})}{2 s b} \xi^{2}+O\left(\frac{\sigma \xi}{b\left\|\boldsymbol{v}_{t}\right\|}+\min \left\{1, \frac{\sigma^{2} \xi^{2}}{b s}\right\}\right) .
\end{aligned}
$$

En reportant dans $(5 \cdot 11)$ puis $(5 \cdot 10)$, nous obtenons que la quantité $\Phi^{*}$ à majorer vérifie

$$
\Phi^{*} \ll \sigma \xi \Phi_{1}^{*}+\Phi_{2}^{*}
$$

où l'on a posé

$$
\Phi_{1}^{*}:=\sum_{b \leqslant y} \sum_{t \leqslant y / b} \sum_{\mathcal{A} \in \mathcal{U}_{T}(b t)} \frac{1}{b\left\|\boldsymbol{v}_{t}(\mathcal{A})\right\|}, \quad \Phi_{2}^{*}:=\sum_{s \leqslant y} \frac{\varrho_{T}^{*}(s)}{\varphi(s)} \sum_{\substack{b \leqslant y \\ b \mid s}} \min \left\{1, \frac{\sigma^{2} \xi^{2}}{b s}\right\} .
$$

Maintenant,

$$
\begin{aligned}
\Phi_{1}^{*} & \leqslant \sum_{b \leqslant y} \frac{1}{b} \sum_{\substack{\boldsymbol{v} \in\left(\mathbb{Z}^{2}\right)^{*} \\
\|\boldsymbol{v}\|^{2} \leqslant 2 y / b}} \frac{1}{\|\boldsymbol{v}\|} \sum_{\substack{\|\boldsymbol{v}\|^{2} / 4 \leqslant t \leqslant y / b \\
t \mid T(\boldsymbol{v})}} 1 \\
& \leqslant \sum_{b \leqslant y} \frac{1}{b} \sum_{\substack{\boldsymbol{v} \in\left(\mathbb{Z}^{2}\right)^{*} \\
\|\boldsymbol{v}\|^{2} \leqslant 2 y / b}} \frac{\tau(T(\boldsymbol{v}))}{\|\boldsymbol{v}\|} \ll\|T\|^{\varepsilon} \sqrt{y} \log \xi,
\end{aligned}
$$

où nous avons successivement fait appel au Théorème 4.1, au Lemme 4.3 et à la majoration

$$
E_{J}(\sqrt{y} ; \tau) \ll \log \xi .
$$

Cette majoration de $\Phi_{1}^{*}$ sera affinée en reportant (5·6) dans (5·15). 
La contribution $\Phi_{21}^{*}$ à $\Phi_{2}^{*}$ des entiers $s$ tels que $b s>\sigma^{2} \xi^{2}$ vérifie

$$
\begin{aligned}
\Phi_{21}^{*} & \ll_{\varepsilon}\|T\|^{\varepsilon / 3} \sigma^{2} \xi^{2} \sum_{b>(\sigma \xi)^{2} / y} \frac{\varrho_{T}^{*}(b)}{\varphi(b) b^{2}} \sum_{\sigma^{2} \xi^{2} / b^{2}<t \leqslant y / b} \frac{\varrho_{T}^{*}(t)}{\varphi(t) t} \\
& \ll_{\varepsilon}\|T\|^{2 \varepsilon / 3} \sigma^{2} \xi^{2} \sum_{b \geqslant \sigma \xi / \sqrt{y}} \frac{\varrho_{T}^{*}(b)}{\varphi(b) b^{2}} \log \left(2+\frac{y b}{\sigma^{2} \xi^{2}}\right) \\
& \ll_{\varepsilon}\|T\|^{\varepsilon} \sigma \xi \min (\sqrt{y}, \sigma \xi) \log \left(2+\frac{y}{\sigma^{2} \xi^{2}}\right),
\end{aligned}
$$

où les sommes relatives à $\varrho_{T}^{*}$ ont été majorées en utilisant $(3 \cdot 8),(3 \cdot 9)$ et les propriétés de $\zeta_{k}(s)$, en tenant compte du fait que le corps $k$ est indépendant de $q$ et $M$. Nous avons également utilisé la sous-multiplicativité faible de la fonction $n \mapsto \varrho_{T}^{*}(n) / \varphi(n)$ sous la forme

$$
\frac{\varrho_{T}^{*}(b t)}{\varphi(b t)} \ll_{\varepsilon}\|T\|^{\varepsilon / 3} \frac{\varrho_{T}^{*}(b) \varrho_{T}^{*}(t)}{\varphi(b) \varphi(t)} \quad(\varepsilon>0) .
$$

Lorsque $(b t, T(1,0) \mathcal{D}(T))=1$, cette majoration découle du Lemme 3.2 et de l'inégalité $\varrho_{T}^{-}(m n) \leqslant \varrho_{T}^{-}(m) \varrho^{-}(n)(m \geqslant 1, n \geqslant 1)$; lorsque $(b, t, T(1,0) \mathcal{D}(T))>1$, il suffit de faire appel à $(3 \cdot 8)$ et $(3 \cdot 9)$.

La contribution $\Phi_{22}^{*}$ à $\Phi_{2}^{*}$ des entiers $s$ tels que $b s \leqslant \sigma^{2} \xi^{2}$ vérifie

$$
\begin{aligned}
\Phi_{22}^{*} & \ll \sum_{b \geqslant 1} \frac{\varrho_{T}^{*}(b)}{\varphi(b)} \sum_{t \leqslant \min \left(y / b, \sigma^{2} \xi^{2} / b^{2}\right)} \frac{\varrho_{T}^{*}(t)}{\varphi(t)} \ll\|T\|^{\varepsilon / 2} \sum_{b \geqslant 1} \frac{\varrho_{T}^{*}(b)}{\varphi(b) b} \min \left(y, \sigma^{2} \xi^{2} / b\right) \\
& \ll\|T\|^{\varepsilon} \min \left(y, \sigma^{2} \xi^{2}\right) \log \left(2+\frac{\sigma^{2} \xi^{2}}{y}\right) .
\end{aligned}
$$

En reportant les deux dernières estimations dans $(5 \cdot 14)$, nous obtenons

$$
\Phi_{2}^{*} \ll\|T\|^{\varepsilon} \sigma \xi \min (\sqrt{y}, \sigma \xi) \log _{2} \xi, \quad \Phi^{*} \ll\|T\|^{\varepsilon} \sqrt{y} \sigma \xi \log \xi .
$$

L'étape suivante de la démonstration consiste à déduire la majoration de $\Phi$ de celle de $\Phi^{*}$. Rappelons à cet effet que nous nous sommes placés dans l'hypothèse où $T$ est primitive. D'après $(5 \cdot 8)$, nous avons

$$
\left|\Lambda(d ; T) \cap \mathcal{R}^{\dagger}(\xi)\right|=\sum_{b \mid \psi(d)}\left|\Lambda^{*}\left(d_{b} ; T\right) \cap \mathcal{R}^{\dagger}(\xi / b)\right|, \quad \varrho_{T}^{+}(d)=\sum_{b \mid \psi(d)} \frac{\varrho_{T}^{*}\left(d_{b}\right) d^{2}}{b^{2} d_{b}^{2}} .
$$

D'où

$$
\Phi \leqslant \sum_{\substack{1 \leqslant d \leqslant y \\ 2 \nmid d}} \sum_{b \mid \psi(d)} \sup _{\mathcal{R}}|| \Lambda^{*}\left(d_{b} ; T\right) \cap \mathcal{R}^{\dagger}(\xi / b)\left|-\frac{\varrho_{T}^{*}\left(d_{b}\right) \operatorname{vol}(\mathcal{R}) \xi^{2}}{2 b^{2} d_{b}^{2}}\right| .
$$

Or, la condition $d \mid b^{4} T(\boldsymbol{x})$ équivaut à $d_{b} \mid T(\boldsymbol{x})$ avec $d_{b}=d /\left(d, b^{4}\right)$. Lorsque $s$ et $b$ sont fixés, il y a au plus $\tau\left(b^{4}\right)$ entiers $d$ tels que $s=d_{b}$ et $b \mid \psi(d)$. De plus, pour chacun de ces entiers $d$, on a $d_{b}=d /\left(d, b^{4}\right) \leqslant d / b \leqslant y / b$. Il suit

$$
\begin{aligned}
\Phi & \leqslant \sum_{b \leqslant y} \tau\left(b^{4}\right) \sum_{\substack{1 \leqslant s \leqslant y / b \\
2 \nmid s}} \sup _{\mathcal{R}}|| \Lambda^{*}(s ; T) \cap \mathcal{R}^{\dagger}(\xi / b)\left|-\frac{\varrho_{T}^{*}(s) \operatorname{vol}(\mathcal{R}) \xi^{2}}{2 s^{2} b^{2}}\right| \\
& \ll \sum_{b \leqslant y} \tau\left(b^{4}\right) \Phi^{*}(\xi / b, y / b ; T, \sigma, \vartheta) .
\end{aligned}
$$


En utilisant la majoration triviale (5.7) lorsque $b \geqslant B:=2+\sigma^{2} \xi^{2} / y$, nous obtenons, pour toute constante $A>0$,

$$
\begin{aligned}
\Phi & \ll\|T\|^{\varepsilon} \sum_{b \leqslant B} \frac{\tau\left(b^{4}\right)}{b}\left(\frac{\sigma \sqrt{y} \xi \log \xi}{\sqrt{b}}+y\right)+\|T\|^{\varepsilon} \sum_{B<b \leqslant y} \frac{\tau\left(b^{4}\right)}{b^{2}} \sigma^{2} \xi^{2} \log (\xi y) \\
& \ll\|T\|^{\varepsilon}\left\{\sigma \xi \sqrt{y} \log \xi+y(\log B)^{5}+\frac{\sigma^{2} \xi^{2} \log (\xi y)(\log B)^{4}}{B}\right\} .
\end{aligned}
$$

Si $y \leqslant \sigma^{2} \xi^{2}$, la somme du second et du troisième terme est

$$
\ll\|T\|^{\varepsilon} \sigma \xi \sqrt{y} \log (\xi y) \cdot \frac{\sqrt{y}}{\sigma \xi}\left(\log \left(2+\frac{\sigma^{2} \xi^{2}}{y}\right)\right)^{5} \ll\|T\|^{\varepsilon} \sigma \xi \sqrt{y} \log (\xi y) .
$$

Compte tenu de $(5 \cdot 7)$, cela fournit

$$
\Phi \ll\|T\|^{\varepsilon} \sigma \xi \min \{\sqrt{y}, \sigma \xi\} \log (\xi y)
$$

ce qui constitue une première étape dans la preuve de $(5 \cdot 5)$.

Nous sommes à présent en mesure d'établir $(5 \cdot 6)$. À cette fin, nous majorons la contribution à la somme de (5.4) de l'ensemble des entiers $d \leqslant y_{1}:=\sigma^{2} \xi^{2} /(\log \xi)^{2}$ en appliquant $(5 \cdot 19)$ avec $y=y_{1}$ : nous obtenons bien une majoration compatible avec $(5 \cdot 6)$.

Traitons à présent la contribution résiduelle, correspondant donc à la sous-somme de (5.6) définie par la condition supplémentaire $y_{1}<d \leqslant y$. Comme $\operatorname{vol}(\mathcal{R}) \leqslant 4 \sigma^{2}$ sous nos hypothèses, nous avons, d'après $(3 \cdot 10)$,

$$
\operatorname{vol}(\mathcal{R}) \xi^{2} \sum_{y_{1}<d \leqslant y} \frac{\varrho_{T}^{+}(d)}{d^{2}} \ll\|T\|^{\varepsilon} \sigma^{2} \xi^{2}\left\{\log _{2} \xi+\log ^{+}\left(y / \sigma^{2} \xi^{2}\right)\right\} .
$$

En appliquant la majoration $\log ^{+} a \ll\left(1+a^{2} / b^{2}\right)\left(1+\log ^{+} b\right)(a>0, b>0)$ pour $a:=y / \sigma^{2} \xi^{2}, b:=y / \vartheta^{2} \xi^{2}$, nous obtenons que l'estimation (5·20) est également compatible avec $(5 \cdot 6)$.

Il reste donc à évaluer

$$
\Theta(\xi):=\sum_{\substack{y_{1}<d \leqslant y \\ 2 \nmid d}}\left|\Lambda(d ; T) \cap \mathcal{R}^{\dagger}(\xi)\right|
$$

$\grave{A}$ cette fin, nous scindons le domaine $\mathcal{R}$ et posons

$$
\mathcal{R}^{\ddagger}(\xi):=\left\{\boldsymbol{x} \in \mathcal{R}^{\dagger}(\xi):|T(\boldsymbol{x})|>\vartheta^{4} \xi^{4} /(\log \xi)^{4 / \kappa},\|\boldsymbol{x}\| \leqslant \sigma \xi\right\}, \quad \mathcal{R}^{*}(\xi):=\mathcal{R}^{\dagger}(\xi) \backslash \mathcal{R}^{\ddagger}(\xi),
$$

de sorte que

$$
\Theta(\xi) \leqslant \Theta^{*}(\xi)+\Theta^{\ddagger}(\xi)
$$

où l'on a posé

$$
\Theta^{*}(\xi):=\sum_{\substack{y_{1} \leqslant d \leqslant y \\ 2 \nmid d}}\left|\Lambda^{*}(d) \cap \mathcal{R}^{*}(\xi)\right|, \quad \Theta^{\ddagger}(\xi):=\sum_{\substack{y_{1} \leqslant d \leqslant y \\ 2 \nmid d}}\left|\Lambda^{*}(d) \cap \mathcal{R}^{\ddagger}(\xi)\right| .
$$


Nous avons

$$
\Theta^{*}(\xi) \leqslant \sum_{x \in \mathcal{R}^{*}(\xi)} \tau(T(\boldsymbol{x})) \leqslant\left(\sum_{x \in \mathcal{R}^{\dagger}(\xi)} \tau(T(\boldsymbol{x}))^{2} \sum_{x \in \mathcal{R}^{*}(\xi)} 1\right)^{1 / 2} .
$$

Un emploi successif du Théorème 4.1 et du Lemme 4.3 permet de montrer que la première somme du membre de droite est

$$
\ll\|T\|^{\varepsilon} \sigma^{2} \xi^{2} E_{J}\left(\sigma \xi ; \tau^{2}\right) \ll\|T\|^{\varepsilon} \sigma^{2} \xi^{2}(\log \xi)^{3},
$$

d'où

$$
\Theta^{*}(\xi) \ll\|T\|^{\varepsilon} \sigma \xi\left|\mathcal{R}^{*}(\xi)\right|^{1 / 2}(\log \xi)^{3 / 2} .
$$

L'estimation de $\left|\mathcal{R}^{*}(\xi)\right|$ résulte de la validité, pour tout $\beta \leqslant 1$, de la majoration

$$
\operatorname{vol}\left\{\boldsymbol{x} \in \mathcal{R}:|T(\boldsymbol{x})| / \vartheta^{4} \leqslant \beta\right\} \leqslant \frac{\sqrt{q}}{\operatorname{dét} M} \operatorname{vol}\left\{\boldsymbol{x} \in \mathcal{R}:|J(\boldsymbol{x})| / \vartheta^{4} \leqslant \beta\right\} \ll_{J} \vartheta^{2} \sqrt{q \beta} .
$$

Pour établir cela, nous appliquons le lemme 3.4 de [15], en vertu duquel

$$
\min _{J(\zeta, 1)=0}|m-\zeta n| \leqslant|J(\boldsymbol{x})| /\|\boldsymbol{x}\|^{3} \quad\left(\boldsymbol{x} \in\left(\mathbb{R}^{2}\right)^{*}\right) .
$$

Il s'ensuit que l'ensemble $\left\{\boldsymbol{x} \in \mathcal{R}:|J(\boldsymbol{x})| / \vartheta^{4} \leqslant \beta\right\}$ est inclus dans

$$
\left\{\|\boldsymbol{x}\| \leqslant \vartheta \beta^{1 / 4}\right\} \bigcup \bigcup_{\substack{(\zeta, 1)=0 \\ j \geqslant 1}}\left\{\boldsymbol{x} \in \mathbb{R}^{2}: 2^{j-1}<\frac{\|x\|}{\vartheta \beta^{1 / 4}} \leqslant 2^{j},|m-\zeta n| \ll \frac{\vartheta \beta^{1 / 4}}{8^{j}}\right\},
$$

qui est de mesure $\ll_{J} \vartheta^{2} \sqrt{\beta}$. Cela établit bien (5·24), d'où, en choisissant $\beta:=1 /(\log \xi)^{4 / \kappa}$,

$$
\left|\mathcal{R}^{*}(\xi)\right| \ll \xi^{2} \operatorname{vol}\left\{\boldsymbol{x} \in \mathcal{R}:|T(\boldsymbol{x})| / \vartheta^{4} \leqslant 1 /(\log \xi)^{4 / \kappa}\right\} \ll \sqrt{q} \vartheta^{2} \xi^{2} /(\log \xi)^{2 / \kappa} .
$$

Compte tenu de $(5 \cdot 23)$ et de l'hypothèse $q \leqslant(\log \xi)^{1 / \kappa}$, il suit, quitte à supposer sans perte de généralité que $\kappa \leqslant \frac{1}{2}$,

$$
\Theta^{*}(\xi) \ll \frac{\|T\|^{\varepsilon} \sigma \vartheta \xi^{2} q^{1 / 4}}{(\log \xi)^{1 / \kappa-3 / 2}} \ll\|T\|^{\varepsilon}(\sigma+\vartheta)^{2} \xi^{2}
$$

une majoration également compatible avec $(5 \cdot 6)$.

Il reste donc à évaluer $\Theta^{\ddagger}(\xi)$. Nous avons

$$
\Theta^{\ddagger}(\xi) \leqslant \sum_{b \leqslant \sigma \xi} \sum_{y_{1}<d \leqslant y} \sum_{\substack{x \in \mathcal{R}^{\ddagger}(\xi / b),\|x\| \leqslant \sigma \xi / b \\(m, n)=1, d \mid b^{4} T(x)}} 1 .
$$

Or, la condition $d \mid b^{4} T(\boldsymbol{x})$ équivaut à $d_{b} \mid T(\boldsymbol{x})$ avec $d_{b}=d /\left(d, b^{4}\right)$. Lorsque $s$ et $b$ sont fixés, il y a au plus $\tau\left(b^{4}\right)$ entiers $d$ tels que $s=d_{b}$. Il suit

$$
\Theta^{\ddagger}(\xi) \leqslant \sum_{b \leqslant \sigma \xi} \tau\left(b^{4}\right) \sum_{y_{1} / b^{4}<s \leqslant y} \sum_{\substack{x \in \mathbb{Z}^{2},\|x\| \leqslant \sigma \xi / b \\(m, n)=1, s \mid T(x)}} 1 .
$$


Désignons par $\Theta_{1}^{\ddagger}$ la contribution au membre de droite de $(5 \cdot 26)$ correspondant à la condition supplémentaire $s \leqslant\left(\sigma^{2}+\vartheta^{2}\right) \xi^{2} / b^{2}$, et notons $\Theta_{2}^{\ddagger}$ la contribution résiduelle. D'après le Lemme 5.1, la somme intérieure de $(5 \cdot 26)$ est

$$
\ll \frac{\varrho_{T}^{+}(s)}{\varphi(s)}\left\{\frac{\sigma^{2} \xi^{2}}{s b^{2}}+1\right\} \ll \frac{\varrho_{T}^{+}(s)\left(\sigma^{2}+\vartheta^{2}\right) \xi^{2}}{s \varphi(s) b^{2}} \quad\left(s \leqslant\left(\sigma^{2}+\vartheta^{2}\right) \xi^{2} / b^{2}\right) .
$$

D'où, grâce à $(3 \cdot 10)$,

$$
\begin{aligned}
\Theta_{1}^{\ddagger} & \ll\left(\sigma^{2}+\vartheta^{2}\right) \xi^{2} \sum_{b \leqslant \sigma \xi} \frac{\tau\left(b^{4}\right)}{b^{2}} \sum_{y_{1} / b^{4}<s \leqslant\left(\sigma^{2}+\vartheta^{2}\right)} \frac{\xi^{2} \varrho_{T}^{+}(s)}{b^{2} s \varphi(s)} \\
& \ll\|T\|^{\varepsilon / 2}\left(\sigma^{2}+\vartheta^{2}\right) \xi^{2} \sum_{b \leqslant \sigma \xi} \frac{\tau\left(b^{4}\right)}{b^{2}}\{\log (b \log \xi)+\log (1+\vartheta / \sigma)\} \\
& \ll\|T\|^{\varepsilon}\left(\sigma^{2}+\vartheta^{2}\right) \xi^{2} \log _{2} \xi
\end{aligned}
$$

où nous avons utilisé la majoration $\vartheta \leqslant\|T\|^{1 / 4} \sigma$.

Pour estimer $\Theta_{2}^{\ddagger}$, nous introduisons $\ell:=T(\boldsymbol{x}) / s \leqslant(\vartheta \xi / b)^{4} / s \leqslant \vartheta^{4} \xi^{2} /(\sigma b+\vartheta b)^{2}$ et observons que

$$
\ell=T(\boldsymbol{x}) / s>\vartheta^{4} \xi^{4} /\left\{s(\log \xi)^{4 / \kappa}\right\} \geqslant \vartheta^{4} \xi^{4} /\left\{y_{1}(\log \xi)^{4 / \kappa}\right\}
$$

Il vient donc, par un nouveau recours au Lemme 5.1,

$$
\begin{aligned}
\Theta_{2}^{\ddagger} & \ll \sum_{b \leqslant \sigma \xi} \tau\left(b^{4}\right) \sum_{\vartheta^{4} \xi^{4} /\left\{y_{1}(\log \xi)^{4 / \kappa}\right\}<\ell \leqslant \vartheta^{4} \xi^{2} /(\sigma b+\vartheta b)^{2}} \frac{\varrho_{T}^{+}(\ell)}{\varphi(\ell)}\left\{\frac{\sigma^{2} \xi^{2}}{\ell b^{2}}+1\right\} \\
& \ll(\vartheta+\sigma)^{2} \xi^{2} \sum_{b \leqslant \sigma \xi} \frac{\tau\left(b^{4}\right)}{b^{2}} \sum_{\vartheta^{4} \xi^{4} /\left\{y_{1}(\log \xi)^{4 / \kappa}\right\}<\ell \leqslant \vartheta^{4} \xi^{2} /(\sigma b+\vartheta b)^{2}} \frac{\varrho_{T}^{+}(\ell)}{\varphi(\ell) \ell} \\
& \ll\|T\|^{\varepsilon}(\vartheta+\sigma)^{2} \xi^{2} \sum_{b \leqslant \sigma \xi} \frac{\tau\left(b^{4}\right)}{b^{2}}\left\{\log (b \log \xi)+\log ^{+}\left(y_{1} /\left(\sigma^{2}+\vartheta^{2}\right) \xi^{2}\right)\right\} \\
& \ll\|T\|^{\varepsilon}(\vartheta+\sigma)^{2} \xi^{2} \log _{2} \xi .
\end{aligned}
$$

Cela achève la démonstration de $(5 \cdot 6)$.

La même majoration est valable pour la quantité $\Phi^{*}$ définie en $(5 \cdot 9)$, soit

$$
\Phi^{*}(\xi, y ; T, \sigma, \vartheta) \ll\|T\|^{\varepsilon}(\sigma+\vartheta)^{2} \xi^{2} \log _{2} \xi .
$$

Pour établir cela, nous observons d'abord que la contribution à (5.9) des entiers $s \leqslant y_{1}$ peut être estimée par (5·17). Ensuite, nous majorons trivialement, pour $\left.s \in] y_{1}, y\right]$, le terme général de (5.9) par l'inégalité triangulaire. Comme

$$
\frac{\varrho_{T}^{*}(s) \operatorname{vol}(\mathcal{R}) \xi^{2}}{2 s^{2}} \leqslant \frac{\varrho_{T}^{+}(s) \operatorname{vol}(\mathcal{R}) \xi^{2}}{2 s^{2}},
$$

la contribution correspondant à la somme sur $\left.s \in] y_{1}, y\right]$ peut être majorée comme dans $(5 \cdot 20)$. De plus l'inégalité

$$
\left|\Lambda^{*}(s ; T) \cap \mathcal{R}^{\dagger}(\xi)\right| \leqslant\left|\Lambda(s ; T) \cap \mathcal{R}^{\dagger}(\xi)\right|
$$

induit à la même somme une contribution $\leqslant \Theta(\xi)$, une quantité précedemment majorée de manière satisfaisante. 
D'après (5·12), les vecteurs $\boldsymbol{v}_{t}=\boldsymbol{v}_{t}(\mathcal{A})$ apparaissant dans (5·15) vérifient $\left\|\boldsymbol{v}_{t}\right\|^{2} \leqslant 2 y / b$. Lorsque $\left.\left.\left\|\boldsymbol{v}_{t}\right\| / \sqrt{2 y / b} \in\right] 1 / 2^{j+1}, 1 / 2^{j}\right]$, on a $t>y /\left(b 2^{2(j+1)}\right)$. En reportant dans la première définition $(5 \cdot 15)$, il suit

$$
\Phi_{1}^{*} \ll \sum_{b \leqslant y} \frac{1}{b} \sum_{j \geqslant 0} \sum_{y /\left(b 2^{2(j+1)}\right)<t \leqslant y / b} \frac{2^{j}}{\sqrt{y / b}} \sum_{\substack{\boldsymbol{v} \in\left(\mathbb{Z}^{2}\right)^{*} \\\|\boldsymbol{v}\| \leqslant \sqrt{2 y / b} / 2^{j} \\ t \mid T(\boldsymbol{v})}} 1 .
$$

Appliquons alors le lemme 2.2 de [31] sous la forme $E_{J}(\sigma \xi ; \Delta(\cdot, 1)) \ll \mathcal{L}(\sigma \xi)^{\sqrt{2}+\varepsilon}$. En utilisant en outre le Théorème 4.1 et le Lemme 4.3, nous obtenons

$$
\Phi_{1}^{*} \ll \sum_{b \leqslant y} \frac{1}{b} \sum_{j \geqslant 0} \frac{(j+1) 2^{j}}{\sqrt{y / b}} \sum_{\substack{\boldsymbol{v} \in\left(\mathbb{Z}^{2}\right)^{*} \\\|\boldsymbol{v}\| \leqslant \sqrt{2 y / b} / 2^{j}}} \Delta(T(\boldsymbol{v}), 1) \ll\|T\|^{\varepsilon} \sqrt{y} \mathcal{L}(\sigma \xi)^{\sqrt{2}+\varepsilon},
$$

puis

$$
\Phi^{*} \ll\|T\|^{\varepsilon}(\sigma \xi \sqrt{y}+y) \mathcal{L}(\sigma \xi)^{\sqrt{2}+\varepsilon} .
$$

En reportant finalement dans $(5 \cdot 18)$ et en faisant appel à $(5 \cdot 28)$ pour les grandes valeurs de $b$, il vient, uniformément par rapport au paramètre $B \geqslant 2$,

$$
\begin{aligned}
\frac{\Phi}{\|T\|^{\varepsilon} \mathcal{L}(\sigma \xi)^{\sqrt{2}+\varepsilon}} & \ll \sum_{b \leqslant B} \frac{\tau\left(b^{4}\right)}{b}\left(\frac{\sigma \xi \sqrt{y}}{b^{1 / 2}}+y\right)+\sum_{B<b \leqslant y} \frac{\tau\left(b^{4}\right)}{b^{2}}(\sigma+\vartheta)^{2} \xi^{2} \\
& \ll\left\{\sigma \xi \sqrt{y}+y(\log B)^{5}+(\log B)^{4}(\sigma+\vartheta)^{2} \xi^{2} / B\right\},
\end{aligned}
$$

puisque le terme $\log _{2} \xi$ apparaissant dans le majorant de (5·28) est négligeable devant $\mathcal{L}(\sigma \xi)^{\sqrt{2}+\varepsilon}$. En choisissant $B=2+(\vartheta+\sigma)^{2} \xi^{2} / y$, nous obtenons le résultat annoncé.

Comme annoncé dans la remarque (i) suivant l'énoncé du Lemme 5.2, indiquons à présent comment remplacer, dans le majorant de $(5 \cdot 5)$, le facteur $\mathcal{L}(\sigma \xi)^{\sqrt{2}+\varepsilon}$ par $\|T\|^{1 / 2}\left(\log _{2} \xi\right)^{2}$. Au vu de ce qui précède, il suffit d'établir la majoration

$$
\Phi_{1}^{*} \ll\|T\|^{1 / 2+\varepsilon} \sqrt{y}\left(\log _{2} \xi\right)^{2} .
$$

Rappelons la définition de $\Phi_{1}^{*}$ en $(5 \cdot 15)$. Les vecteurs $\boldsymbol{v}_{t}=\boldsymbol{v}_{t}(\mathcal{A})$ y apparaissant ont au moins une coordonnée impaire. La variante de (5.29) où les vecteurs $\boldsymbol{v}=\left(v_{1}, v_{2}\right)$ satisfont à cette même condition est donc valide. La contribution au membre de droite des vecteurs $\boldsymbol{v}$ tels que $v_{1}$ est impair est

$$
\ll \sum_{b \leqslant y} \frac{1}{b} \sum_{j \geqslant 1} \frac{2^{j}}{\sqrt{2 y / b}}\left\{\Phi\left(\frac{\sqrt{2 y / b}}{2^{j}}, \frac{y}{b} ; T, 1,\|T\|^{1 / 4}\right)+\sum_{y /\left(b 2^{2(j+1)}\right) \leqslant s \leqslant y / b} \frac{y \varrho_{T}^{+}(s)}{4^{j} b s^{2}}\right\} .
$$

En appliquant $(5 \cdot 6)$ et en faisant appel à $(3 \cdot 10)$, nous obtenons alors que cette quantité est dominé par le majorant de (5·32). La contribution complémentaire peut être estimée similairement. 


\section{Sommes de carrés localisées : majorations}

Cette section est dévolue à l'obtention de majorations pour les quantités $\mathfrak{S}(\xi, d ; T)$ et $S_{T}(\xi, d)$ respectivement définies en $(2 \cdot 3)$ et $(2 \cdot 5)$.

Lemme 6.1. Soient $T \in \mathbb{Z}[X, Y]$ une forme binaire de degré 4 , irréductible sur $\mathbb{Q}[i]$, et $\varepsilon>0$. Sous les conditions $\xi \geqslant 1, d \geqslant 1, \mu^{2}(d)=1$, nous avons uniformément

$$
\begin{aligned}
& \mathfrak{S}(\xi, d ; T) \ll d^{\varepsilon}\left(\frac{\xi^{2}}{d}+\xi^{1+\varepsilon}\right), \\
& S_{T}(\xi, d) \ll d^{\varepsilon}\left(\frac{\xi^{2}}{d}+\xi^{1+\varepsilon}\right) .
\end{aligned}
$$

Les constantes implicites dépendent au plus de la forme binaire $T$ et de $\varepsilon$.

Démonstration. Observons tout d'abord que (6·2) découle facilement de (6·1). En effet, nous avons, avec les notations $(2 \cdot 8)$,

$$
\mathfrak{S}\left(\xi, d_{\ell} ; T_{\ell}\right) \ll \ell^{\varepsilon} \mathfrak{S}\left(\xi, d_{\ell} ; T\right)
$$

et la relation $(2 \cdot 7)$ fournit immédiatement l'estimation souhaitée.

Il suffit donc de montrer $(6 \cdot 1)$. D'après $(5 \cdot 8)$, nous pouvons écrire, avec les notations introduites au paragraphe 5.1 et en notant que $\psi(d)=d$ puisque $\mu(d)^{2}=1$,

$$
\Lambda(d ; T) \subset \bigcup_{b \mid d} \bigcup_{\mathcal{A} \in \mathcal{U}_{T}\left(d_{b}\right)} b \mathcal{A}_{d_{b}}
$$

où $\mathcal{A}_{d_{b}}$ est un réseau de $\mathbb{Z}^{2}$ de rang 2 et de déterminant $d_{b}=d /(d, b)$. D'où

$$
\begin{aligned}
\mathfrak{S}(\xi, d ; T) & \leqslant \sum_{b \mid d} \sum_{\mathcal{A} \in \mathcal{U}_{T}\left(d_{b}\right)} \sum_{\boldsymbol{x} \in \mathcal{R}_{T}(\xi) \cap b \mathcal{A}_{d_{b}}} r(T(\boldsymbol{x}) / d) \\
& \leqslant \sum_{b \mid d} \sum_{\mathcal{A} \in \mathcal{U}_{T}\left(d_{b}\right)} \sum_{x \in \mathcal{R}_{T}(\xi / b) \cap \mathcal{A}_{d_{b}}} r\left(b^{4} T(\boldsymbol{x}) / d\right) .
\end{aligned}
$$

Pour chaque $s=d_{b}$, choisissons une base minimale $\left(\boldsymbol{v}_{s}, \boldsymbol{w}_{s}\right)$ du réseau $\mathcal{A}_{s}$ satisfaisant aux propriétés $(5 \cdot 12),(5 \cdot 13)$. En particulier, la condition $\boldsymbol{x}=\lambda \boldsymbol{v}_{s}+\nu \boldsymbol{w}_{s} \in \mathcal{R}_{T}(\xi / b)$ avec $(\lambda, \nu) \in \mathbb{Z}^{2}$ implique

$$
|\lambda| \leqslant 2 \delta_{\infty}\left(\mathcal{R}_{T}\right) \xi /\left\{b\left\|\boldsymbol{v}_{s}\right\|\right\}, \quad|\nu| \leqslant 2 \delta_{\infty}\left(\mathcal{R}_{T}\right) \xi /\left\{b\left\|\boldsymbol{w}_{s}\right\|\right\}
$$

Considérons le polynôme

$$
T_{s, b}(\lambda, \nu):=b^{4} T\left(\lambda \boldsymbol{v}_{s}+\nu \boldsymbol{w}_{s}\right) / d
$$

dont les coefficients sont $\ll b^{4} s^{3}$, et appliquons le Théorème 4.1 avec

$$
u:=2 \delta_{\infty}\left(\mathcal{R}_{T}\right) \xi /\left\{b\left\|\boldsymbol{v}_{s}\right\|\right\}, \quad x:=2 \delta_{\infty}\left(\mathcal{R}_{T}\right) \xi /\left\{b\left\|\boldsymbol{w}_{s}\right\|\right\}
$$


Lorsque $\min (u, x) \leqslant \xi^{\varepsilon / 2}$, nous avons

$$
\sum_{x \in \mathcal{R}(\xi / b) \cap \mathcal{A}_{s}} r\left(b^{4} T(\boldsymbol{x}) / d\right) \ll \xi^{\varepsilon / 2} \max (u, x) \max _{1 \leqslant|n| \ll \xi^{4}} r(n) \ll \xi^{1+\varepsilon} .
$$

La contribution correspondante au membre de gauche de $(6 \cdot 3)$ est donc

$$
\ll \xi^{1+\varepsilon} \sum_{b \mid d} \frac{\varrho_{T}^{*}\left(d_{b}\right)}{\varphi\left(d_{b}\right)} \ll \xi^{1+\varepsilon} d^{\varepsilon} .
$$

Lorsque $\min (u, x)>\xi^{\varepsilon / 2}$, nous désignons par $q$ le contenu de $T_{s, b}$, de sorte que $T_{s, b}^{*}:=T_{s, b}(\boldsymbol{x}) / q$ est primitif et nous introduisons la fonction multiplicative $r_{q}$ définie sur l'ensemble des puissances de nombres premiers par

$$
r_{q}\left(p^{\nu}\right)= \begin{cases}\nu+1 & \text { si } p \mid q, \\ r\left(p^{\nu}\right) & \text { si } p \nmid q .\end{cases}
$$

Nous avons donc

$$
r(q n) \leqslant \tau(q) r_{q}(n) \quad\left(n \in \mathbb{N}^{*}\right) .
$$

Observons de plus que $\varrho_{T_{s, b}^{*}}^{-}(p)=\varrho_{T}^{-}(p)$ lorsque $p \nmid b q$ et que $\mathcal{D}\left(T_{s, b}^{*}\right) \mid \mathcal{D}(T)$. Grâce au Théorème 4.1 et au Lemme 3.3, nous obtenons dans ce cas

$$
\begin{aligned}
\sum_{x \in \mathcal{R}(\xi / b) \cap \mathcal{A}_{s}} r\left(b^{4} T(\boldsymbol{x}) / d\right) & \leqslant \tau(q) \sum_{\lambda \leqslant x, \nu \leqslant u} r_{q}\left(T_{s, b}^{*}(\lambda, \nu)\right) \\
& \ll_{T, \varepsilon} u x d^{\varepsilon / 2} \exp \left(\sum_{p \leqslant u+x} \frac{\chi(p) \varrho_{T}^{-}(p)}{p}\right) \ll d^{\varepsilon / 2} \frac{\xi^{2}}{b^{2} t} .
\end{aligned}
$$

En reportant cette estimation dans (6.3) et en observant que

$$
\sum_{b \mid d} \sum_{\mathcal{A} \in \mathcal{U}_{T}\left(d_{b}\right)} \frac{1}{b^{2} d_{b}}=\sum_{b \mid d} \frac{\varrho_{T}^{*}\left(d_{b}\right)}{b^{2} d_{b} \varphi\left(d_{b}\right)} \ll d^{\varepsilon / 2-1},
$$

nous obtenons le résultat annoncé en $(6 \cdot 1)$.

\section{Sommes de carrés localisées : formules asymptotiques}

\section{\% 1. Énoncé et remarques liminaires}

Nous posons

$$
Q(\lambda)=\lambda \log \lambda-\lambda+1 \quad(\lambda>0)
$$

et notons

$$
\mathcal{E}:=\bigcup_{\mu \geqslant 0}\left\{n \in \mathbb{N}^{*}: n \equiv 2^{\mu}\left(\bmod 2^{\mu+2}\right)\right\} .
$$

Nous nous proposons ici, sous certaines conditions concernant la forme binaire $T$ et le domaine $\mathcal{R}$, d'évaluer asymptotiquement l'expression

$$
\mathcal{Q}(\xi ; T, \mathcal{R}):=\sum_{\boldsymbol{x} \in \mathbb{Z}^{2} \cap \mathcal{R}(\xi)} r(T(\boldsymbol{x}))
$$

dont la quantité $(2 \cdot 3)$ est un cas particulier. Lorsque $\nu \geqslant 0$, nous notons $\mathcal{E}_{\nu}$ l'image de $\mathcal{E}$ par la projection canonique de $\mathcal{E}$ dans $\mathbb{Z} / 2^{\nu} \mathbb{Z}$, soit

$$
\mathcal{E}_{\nu}:=\left\{n \in \mathbb{Z} / 2^{\nu} \mathbb{Z}: \exists \mu \in \mathbb{N}: n \equiv 2^{\mu}\left(\bmod 2^{\min (\mu+2, \nu)}\right)\right\} .
$$


Lemme 7.1. Soient $\varepsilon>0, \kappa>0, J \in \mathbb{Z}[X, Y]$ une forme binaire de degré 4 irréductible sur $\mathbb{Q}[i], M \in M_{2}(\mathbb{Z})^{*}, q \in \mathbb{N}^{*}$, tels que $2 \nmid q$ dét $M$, et $\mathcal{R}$ un domaine de $\mathbb{R}^{2}$ vérifiant $\left(H_{1}\right)$. Sous l'hypothèse que la forme binaire $T$ définie par $T(\boldsymbol{x}):=J(M \boldsymbol{x}) / q\left(\boldsymbol{x} \in \mathbb{R}^{2}\right)$ est à coefficients entiers et vérifie $\left(H_{2}\right)$ et $\left(H_{3}\right)$, et sous les conditions

$$
\begin{aligned}
& \xi \geqslant \mathrm{e}^{\kappa q}+\|M\|^{\kappa}, 1 / \sqrt{\xi} \leqslant \sigma \leqslant \xi^{3 / 2}, \quad 1 / \sqrt{\xi} \leqslant \vartheta \leqslant \xi^{3 / 2}, \\
& \delta_{\infty}(\mathcal{R}) \leqslant \sigma, \quad \delta_{T}(\mathcal{R}) \leqslant \vartheta, \quad \vartheta \leqslant\|T\|^{1 / 4} \sigma,
\end{aligned}
$$

nous avons

$$
\mathcal{Q}(\xi ; T, \mathcal{R})=\pi K(T) \operatorname{vol}(\mathcal{R}) \xi^{2}+O\left(\frac{\|T\|^{\varepsilon} \xi^{2}(\sigma+\vartheta)^{2}}{(\log \xi)^{1 / 48}}\right)
$$

où $K(T):=\prod_{p} K_{p}(T)$ et

$$
\begin{aligned}
& K_{2}(T):=\lim _{\nu \rightarrow \infty} 2^{-2 \nu+1}\left|\left\{\boldsymbol{x} \in\left(\mathbb{Z} / 2^{\nu} \mathbb{Z}\right)^{2}: T(\boldsymbol{x}) \in \mathcal{E}_{\nu}\right\}\right|, \\
& K_{p}(T):=\left(1-\frac{\chi(p)}{p}\right) \sum_{\nu \geqslant 0} \frac{\chi\left(p^{\nu}\right) \varrho_{T}^{+}\left(p^{\nu}\right)}{p^{2 \nu}} \quad(p>2) .
\end{aligned}
$$

La constante implicite dans (7.5) dépend au plus de $\varepsilon, \kappa$ et $J$.

De plus, nous avons pour tout nombre premier $p$

$$
K_{p}(T)=\lim _{\nu \rightarrow \infty} p^{-3 \nu}\left|\left\{(\boldsymbol{x}, s, t) \in\left(\mathbb{Z} / p^{\nu} \mathbb{Z}\right)^{4}: T(\boldsymbol{x}) \equiv s^{2}+t^{2}\left(\bmod p^{\nu}\right)\right\}\right| .
$$

La somme $Q(\xi ; T, \mathcal{R})$ compte le nombre de solutions entières de

$$
s^{2}+t^{2}=T(m, n)
$$

avec $(m, n) \in \mathcal{R}(\xi)$. Un principe général stipule que le terme principal d'une évaluation asymptotique de cette quantité est égal au produit du carré du facteur de dilatation par les densités archimédienne et $p$-adiques associées au problème. ${ }^{(3)}$ Vérifions que la densité archimédienne $K_{\infty}$ est effectivement donnée par la formule $K_{\infty}=\pi \operatorname{vol}(\mathcal{R})$. Reprenons, à cette fin, les calculs effectués au théorème 4 de [2] dans le même contexte. Par symétrie, nous pouvons nous restreindre aux solutions du premier quadrant $s>0, t>0$, en écrivant $K_{\infty}=4 K_{\infty}^{+}$. Nous calculons alors $K_{\infty}^{+}$en paramétrant $t=\sqrt{T(\boldsymbol{x})-s^{2}}$ et en intégrant la forme de Leray, donnée ici par $(2 t)^{-1} \mathrm{~d} s \mathrm{~d} x \mathrm{~d} y$. Compte tenu de la formule

$$
\int_{0}^{\sqrt{T}} \frac{\mathrm{d} s}{\sqrt{T-s^{2}}}=\frac{1}{2} \pi \quad(T>0)
$$

nous obtenons

$$
K_{\infty}^{+}=\int_{\mathcal{R}} \mathrm{d} x \mathrm{~d} y \int_{0}^{\sqrt{T(x, y)}} \frac{\mathrm{d} s}{2 \sqrt{T(x, y)-s^{2}}}=\frac{1}{4} \pi \operatorname{vol}(\mathcal{R})
$$

d'où la valeur annoncée $K_{\infty}$.

En raisonnant comme dans la démonstration du théorème 4 de [2], on peut montrer similairement que les densités $p$-adiques $K_{p}(T)$ associées à l'équation diophantienne $(7 \cdot 8)$ sont bien données par (7·6).

3. On pourra se reporter à la section 2.2.1 de [27]. 


\subsection{Première réduction du problème}

Notre approche consiste à exploiter systématiquement l'identité de convolution

$$
r(t)=4 \sum_{d \mid t} \chi(d) \quad(t \geqslant 1)
$$

avec $t=T(\boldsymbol{x})$, et à estimer les contributions issues de différentes plages pour la variable $d$. Dans cette perspective, ${ }^{(4)}$ il est essentiel de limiter la taille de $d$. À cette fin, nous ferons appel à la symétrie des diviseurs d'un entier $n$ autour de $\sqrt{n}$. Il sera alors commode de disposer de l'information $\chi(T(\boldsymbol{x}))=1$.

L'étape liminaire de la démonstration consiste à scinder la sommation initiale en sous-sommes vérifiant cette condition. En fait, les nouvelles sommations porteront génériquement sur des vecteurs $\boldsymbol{x}=(m, n) \in \mathbb{Z}^{2}$ et des formes $T$ tels que $2 \nmid m$ et $T(\boldsymbol{x}) \equiv m^{4}(\bmod 4)$.

Désignant par $v_{2}$ la valuation 2-adique, nous commençons par scinder la sommation $(7 \cdot 2)$ selon les valeurs de $v_{2}((m, n))$ et $v_{2}(T(\boldsymbol{x}))$, en utilisant systématiquement la relation $r(2 t)=r(t)\left(t \in \mathbb{N}^{*}\right)$. Nous obtenons

$$
\mathcal{Q}(\xi ; T, \mathcal{R})=\sum_{j \geqslant 0} \mathcal{Q}_{1}\left(\xi / 2^{j} ; T, \mathcal{R}\right)
$$

où l'on a posé

$$
\mathcal{Q}_{1}(\xi ; T, \mathcal{R}):=\sum_{\substack{\boldsymbol{x} \in \mathbb{Z}^{2} \cap \mathcal{R}(\xi) \\ 2 \nmid(m, n)}} r(T(\boldsymbol{x}))=\sum_{k \geqslant 0} \sum_{\substack{\boldsymbol{x} \in \mathbb{Z}^{2} \cap \mathcal{R}(\xi) \\ 2 \nmid(m, n) \\ v_{2}(T(\boldsymbol{x}))=k}} r(T(\boldsymbol{x}))
$$

Rappelons que $r(t)=0$ si $t \equiv 3(\bmod 4)$ et analysons la condition

$$
2 \nmid(m, n), \quad v_{2}(T(\boldsymbol{x}))=k, \quad T(\boldsymbol{x}) / 2^{k} \not \equiv 3(\bmod 4),
$$

qui est certainement satisfaite par tous les vecteurs $\boldsymbol{x}$ de la somme intérieure de $(7 \cdot 12)$ pour lesquels $r(T(\boldsymbol{x})) \neq 0$. Comme $v_{2}(T(\boldsymbol{x}))=k$ implique $T(\boldsymbol{x}) \equiv h 2^{k}\left(\bmod 2^{k+2}\right)$ avec $h=1$ ou 3 et que cette dernière éventualité est exclue, nous voyons que (7.13) équivaut à

$$
2 \nmid(m, n), \quad T(\boldsymbol{x}) \equiv 2^{k}\left(\bmod 2^{k+2}\right) .
$$

Soit $\mathcal{W}_{k}$ l'ensemble des solutions dans $\{1\} \times \mathbb{Z} / 2^{k+2} \mathbb{Z}$ de l'équation

$$
T(\boldsymbol{x}) \equiv 2^{k}\left(\bmod 2^{k+2}\right),
$$

identifié avec un système de représentants dans $\{1\} \times\left[0,2^{k+2}\right.$ [. L'ensemble des solutions $\boldsymbol{x}=(m, n)$ de $(7 \cdot 14)$ où $m$ est impair coïncide donc avec l'ensemble des vecteurs $\boldsymbol{x}$ satisfaisant à $\boldsymbol{x} \equiv \operatorname{m\alpha }\left(\bmod 2^{k+2}\right)$ lorsque $\alpha$ décrit $\mathcal{W}_{k}$.

Symétriquement, désignons par $z_{k}$ l'ensemble des solutions dans $\mathbb{Z} / 2^{k+2} \mathbb{Z} \times\{1\}$ de l'équation $T(X, 1) \equiv 2^{k}\left(\bmod 2^{k+2}\right)$ telles que $X \equiv 0(\bmod 2)$, identifié avec un système de représentants dans $\left[0,2^{k+2}\right.$ [. Alors l'ensemble des solutions $\boldsymbol{x}=(m, n)$ de $(7 \cdot 14)$ où $m$ est pair coïncide avec l'ensemble des vecteurs $\boldsymbol{x}$ vérifiant $\boldsymbol{x} \equiv n \boldsymbol{\alpha}\left(\bmod 2^{k+2}\right)$ où $n$ est impair et $\boldsymbol{\alpha}$ décrit $z_{k}$.

4. Voir notamment la formule $(7 \cdot 28)$. 
Pour chaque entier $k \geqslant 0$, les solutions de (7·13) se répartissent donc en deux classes, que nous pouvons paramétrer sous la forme

$$
\begin{array}{lll}
\text { (i) } \quad \boldsymbol{x}=\left(m, \beta m+2^{k+2} n\right) & \left(m \in 2 \mathbb{Z}+1, n \in \mathbb{Z}, \boldsymbol{\alpha}=(1, \beta) \in \mathcal{W}_{k}\right), \\
\text { (ii) } \quad \boldsymbol{x}=\left(\beta n+2^{k+2} m, n\right) & \left(m \in \mathbb{Z}, n \in 2 \mathbb{Z}+1, \boldsymbol{\alpha}=(\beta, 1) \in \mathcal{Z}_{k}\right) .
\end{array}
$$

Posons

$$
U_{\boldsymbol{\alpha}, k}:=\left\{\begin{array}{cc}
\left(\begin{array}{cc}
1 & 0 \\
\beta & 2^{k+2}
\end{array}\right) & \text { si } \alpha=(1, \beta) \in \mathcal{W}_{k} \\
\left(\begin{array}{cc}
\beta & 2^{k+2} \\
1 & 0
\end{array}\right) & \text { si } \alpha=(\beta, 1) \in \mathcal{Z}_{k},
\end{array}\right.
$$

et

$$
T_{\boldsymbol{\alpha}, k}(\boldsymbol{x}):=T\left(U_{\boldsymbol{\alpha}, k} \boldsymbol{x}\right) / 2^{k} \quad\left(\boldsymbol{x} \in \mathbb{Z}^{2}\right) .
$$

Il résulte de ce qui précède que l'ensemble des valeurs $r(T(\boldsymbol{x}))$ satisfaisant la condition $(7 \cdot 13)$ coïncide avec

$$
\left\{r\left(T_{\boldsymbol{\alpha}, k}(\boldsymbol{x})\right): \boldsymbol{x} \in(2 \mathbb{Z}+1) \times \mathbb{Z}, \alpha \in \mathcal{W}_{k} \cup \mathbb{Z}_{k}\right\} .
$$

De plus, nous avons identiquement

$$
T_{\boldsymbol{\alpha}, k}(\boldsymbol{x}) \equiv m^{4}(\bmod 4) \quad\left(\boldsymbol{x}=(m, n) \in(2 \mathbb{Z}+1) \times \mathbb{Z}, k \geqslant 0, \boldsymbol{\alpha} \in \mathcal{W}_{k} \cup Z_{k}\right) .
$$

Nous notons, à fins de référence ultérieure, que

$$
\left\|T_{\boldsymbol{\alpha}, k}\right\| \leqslant 2^{3 k+8}\|T\| \quad\left(k \geqslant 0, \boldsymbol{\alpha} \in \mathcal{W}_{k} \cup \mathcal{Z}_{k}\right),
$$

et que, avec la notation $(7 \cdot 6)$, nous avons

$$
K_{p}\left(T_{\boldsymbol{\alpha}, k}\right)=K_{p}(T) \quad\left(p>2, k \geqslant 0, \boldsymbol{\alpha} \in \mathcal{W}_{k} \cup \mathcal{Z}_{k}\right) .
$$

Posant

$$
\mathcal{R}_{\boldsymbol{\alpha}, k}:=\left\{\boldsymbol{x} \in \mathbb{R}^{2}: U_{\boldsymbol{\alpha}, k} \boldsymbol{x} \in \mathcal{R}\right\},
$$

de sorte que $\operatorname{vol}\left(\mathcal{R}_{\boldsymbol{\alpha}, k}\right)=\operatorname{vol}(\mathcal{R}) / 2^{k+2}$, et

$$
\mathcal{Q}_{2}(\xi ; T, \mathcal{R}):=\sum_{\substack{x \in \mathbb{Z}^{2} \cap \mathcal{R}(\xi) \\ m \equiv 1 \bmod 2}} r(T(\boldsymbol{x})),
$$

nous pouvons donc écrire

$$
\mathcal{Q}_{1}(\xi ; T, \mathcal{R})=\sum_{k \geqslant 0} \sum_{\boldsymbol{\alpha} \in \mathcal{W}_{k} \cup z_{k}} \mathcal{Q}_{2}\left(\xi ; T_{\boldsymbol{\alpha}, k}, \mathcal{R}_{\boldsymbol{\alpha}, k}\right)
$$

ce qui, associé à $(7 \cdot 11)$, constitue notre première réduction du problème.

Notons que, avec la notation $(7 \cdot 6)$, nous avons

$$
K_{2}(T)=\sum_{j \geqslant 0} \sum_{k \geqslant 0} \frac{\left|\mathcal{W}_{k}\right|+\left|Z_{k}\right|}{2^{2 j+k+2}}=\frac{1}{3} \sum_{k \geqslant 0} \frac{\left|\mathcal{W}_{k}\right|+\left|\mathcal{Z}_{k}\right|}{2^{k}} .
$$


En effet, en classant les couples $\boldsymbol{x}$ selon la valeur de $j:=v_{2}((m, n))$, nous obtenons

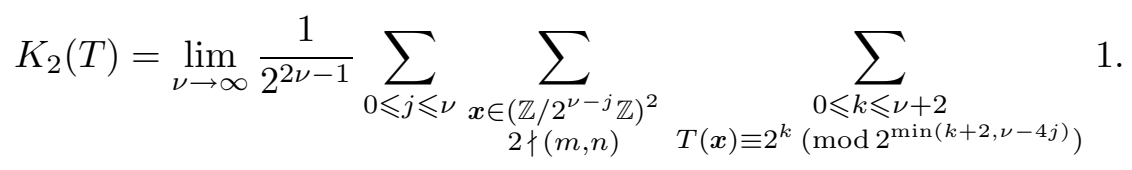

La contribution aux deux sommes intérieures des entiers $k$ tels que $k+2 \geqslant \nu-4 j$ est $\ll \nu 2^{\nu+2 j}$; elle peut donc être négligée dans le passage à la limite. Lorsque $k+2 \leqslant \nu-4 j$, la condition $T(\boldsymbol{x}) \equiv 2^{k}\left(\bmod 2^{k+2}\right)$ ne dépend que de la valeur de $\boldsymbol{x}$ modulo $2^{k+2}$. Il suit

$$
K_{2}(T)=\sum_{j \geqslant 0} \sum_{k \geqslant 0} \frac{1}{2^{2 j+2 k+3}} \sum_{\substack{x \in\left(\mathbb{Z} / 2^{k+2} \mathbb{Z}\right)^{2} \\
2 \nmid(m, n) \\
T(\boldsymbol{x}) \equiv 2^{k}\left(\bmod 2^{k+2}\right)}} 1=\frac{1}{6} \sum_{k \geqslant 0} \frac{1}{2^{2 k}} \sum_{\begin{array}{c}
\boldsymbol{x} \in\left(\mathbb{Z} / 2^{k+2} \mathbb{Z}\right)^{2} \\
2 \nmid(m, n) \\
T(\boldsymbol{x}) \equiv 2^{k}\left(\bmod 2^{k+2}\right)
\end{array}} 1 .
$$

Considérons un vecteur $\boldsymbol{x}=(m, n)$. Si $m$ est impair, alors $\boldsymbol{x}$ est compté dans la somme intérieure si, et seulement si, il existe $\boldsymbol{\alpha}=(1, \beta) \in \mathcal{W}_{k}$ tel que $n \equiv \beta m\left(\bmod 2^{k+2}\right)$; il y a $2^{k+1}\left|\mathcal{W}_{k}\right|$ tels vecteurs $\boldsymbol{x}$ dans $\left(\mathbb{Z} / 2^{k+2} \mathbb{Z}\right)^{2}$. Si $m$ est pair, alors $n$ est impair et $\boldsymbol{x}$ apparaît dans la sommation si, et seulement si, il existe $\boldsymbol{\alpha}=(\beta, 1) \in \mathcal{Z}_{k}$ tel que $m \equiv \beta n\left(\bmod 2^{k+2}\right)$; il y a $2^{k+1}\left|Z_{k}\right|$ tels vecteurs dans $\left(\mathbb{Z} / 2^{k+2} \mathbb{Z}\right)^{2}$. En reportant ces valeurs dans $(7 \cdot 23)$, nous obtenons bien $(7 \cdot 22)$.

Nous aurons ultérieurement l'usage d'une estimation de la vitesse de convergence de la série $(7 \cdot 22)$. À cette fin, nous remarquons que, puisque $2 \nmid q$ dét $M$, nous avons

$$
\left|\mathcal{W}_{k}\right|+\left|\mathcal{Z}_{k}\right| \leqslant \frac{1}{2^{k}} \sum_{\substack{(m, n) \in\left(\mathbb{Z} / 2^{k+2} \mathbb{Z}\right)^{2} \\ 2 \nmid(m, n) \\ T(m, n) \equiv 0\left(\bmod 2^{k}\right)}} 1 \ll \frac{\varrho_{T}^{+}\left(2^{k}\right)}{2^{k}} \ll \frac{\varrho_{J}^{+}\left(2^{k}\right)}{2^{k}} \ll J .
$$

Il suit

$$
\sum_{\substack{(j, k) \in \mathbb{N}^{2} \\ \max (j, k) \geqslant z}} \frac{\left|\mathcal{W}_{k}\right|+\left|z_{k}\right|}{2^{2 j+k+2}} \ll \frac{1}{2^{z}} \quad(z \geqslant 0)
$$

où la constante implicite est indépendante de $M$ et $q$.

\section{\%.3. Seconde réduction}

Dans ce paragraphe, nous nous proposons d'utiliser (7.21) pour établir la formule asymptotique

$$
\mathcal{Q}_{1}(\xi ; T, \mathcal{R})=\frac{3}{4} \pi K(T) \operatorname{vol}(\mathcal{R}) \xi^{2}+O_{\varepsilon}\left(\frac{\|T\|^{\varepsilon} \xi^{2}(\sigma+\vartheta)^{2}}{(\log \xi)^{1 / 48}}\right)
$$

sous les hypothèses du Lemme 7.1 et sous réserve de la validité d'une estimation auxiliaire - cf. (7.41) infra - qui sera explicitée plus loin.

Notons d'emblée que la formule asymptotique $(7 \cdot 5)$ résulte immédiatement de $(7 \cdot 26)$ via $(7 \cdot 11)$.

La validité de la condition $(7 \cdot 16)$ pour les vecteurs $\boldsymbol{x}$ pris en compte par la somme $(7 \cdot 21)$ garantit que $T_{\boldsymbol{\alpha}, k}(\boldsymbol{x}) \equiv 1(\bmod 4)$ et donc $\chi\left(T_{\boldsymbol{\alpha}, k}(\boldsymbol{x})\right)=1$. Donnons-nous alors un paramètre réel $y$ satisfaisant à

$$
\vartheta^{2} \xi^{2} / \log \xi<y<\vartheta^{2} \xi^{2} /(\log \xi)^{\varepsilon},
$$


posons

$$
\left.\left.\left.\mathcal{J}_{1}:=[1, y], \quad \mathcal{J}_{2}:=\right] y, \vartheta^{4} \xi^{4} / y\right], \quad \mathcal{J}_{3}:=\right] \vartheta^{4} \xi^{4} / y, \infty[,
$$

décomposons la somme de $(7 \cdot 10)$ sous la forme

$$
r(t)=\sum_{1 \leqslant j \leqslant 3} r_{j}(t) \quad(t \geqslant 1)
$$

où $r_{j}(t):=4 \sum_{d \mid t, d \in \mathcal{J}_{j}} \chi(d)(1 \leqslant j \leqslant 3)$, et notons immédiatement que, sous la condition $t \equiv 1(\bmod 4)$, nous pouvons remplacer $\mathcal{J}_{3}$ par

$$
\mathcal{J}_{3}(t):=\left[1, y t / \vartheta^{4} \xi^{4}[.\right.
$$

Reportons dans $(7 \cdot 21)$ et, pour $1 \leqslant j \leqslant 3$, désignons par $\mathcal{Q}_{1 j}(\xi ; T, \mathcal{R})$ la contribution globale de $r_{j}$, de sorte que

$$
\mathcal{Q}_{1}(\xi ; T, \mathcal{R})=\sum_{1 \leqslant j \leqslant 3} \mathcal{Q}_{1 j}(\xi ; T, \mathcal{R})
$$

avec donc, pour $j \in\{1,2,3\}$,

$$
\mathcal{Q}_{1 j}(\xi ; T, \mathcal{R})=4 \sum_{k \geqslant 0} \sum_{\boldsymbol{\alpha} \in \mathcal{W}_{k} \cup z_{k}} \sum_{\boldsymbol{x} \in \mathcal{R}_{\boldsymbol{\alpha}, k}(\xi) \cap \mathbb{Z}^{2}} \sum_{\substack{d \mid T_{\boldsymbol{\alpha}, k}(\boldsymbol{x}) \\ d \in \mathcal{J}_{j}\left(T_{\boldsymbol{\alpha}, k}(\boldsymbol{x})\right)}} \chi(d)
$$

où nous convenons que $\mathcal{J}_{j}(t):=\mathcal{J}_{j}$ si $j=1$ ou 2 . Notons que pour $j=1$ ou 2 , nous avons

$$
\mathcal{Q}_{1 j}(\xi ; T, \mathcal{R})=4 \sum_{\substack{x \in \mathcal{R}(\xi) \cap \mathbb{Z}^{2} \\ 2 \nmid(m, n)}} \sum_{\substack{d \mid T(x) \\ d \in \mathcal{J}_{j}}} \chi(d) .
$$

Nous verrons que $\mathcal{Q}_{12}(\xi ; T, \mathcal{R})$ peut être considéré comme un terme d'erreur. Évaluons dans un premier temps les quantités $\mathcal{Q}_{11}(\xi ; T, \mathcal{R})$ et $\mathcal{Q}_{13}(\xi ; T, \mathcal{R})$. Pour cela, nous intervertirons les sommations en $d$ et $\boldsymbol{x}$ dans (7.30) et estimerons ensuite la somme intérieure en $\boldsymbol{x}$ grâce au Lemme 5.2.

La mise en œuvre de ce calcul nécessite de définir et d'estimer dans ce nouveau contexte, uniformément en $\boldsymbol{\alpha}$ et $k$, les différentes quantités apparaissant dans la définition (5·4).

Pour $k \geqslant 0, \boldsymbol{\alpha} \in \mathcal{W}_{k} \cup z_{k}, d \geqslant 1$, nous posons

$$
\begin{aligned}
\mathcal{R}_{1}(d) & :=\mathcal{R}, \quad \mathcal{R}_{3}(d):=\left\{\boldsymbol{x} \in \mathcal{R}: d<y T(\boldsymbol{x}) / \vartheta^{4}\right\}, \\
\mathcal{R}_{1}(d, \boldsymbol{\alpha}, k) & :=\mathcal{R}_{\boldsymbol{\alpha}, k}, \quad \mathcal{R}_{3}(d, \boldsymbol{\alpha}, k):=\left\{\boldsymbol{x} \in \mathcal{R}_{\boldsymbol{\alpha}, k}: d<y T_{\boldsymbol{\alpha}, k}(\boldsymbol{x}) / \vartheta^{4}\right\}, \\
\mathcal{R}_{j}^{\dagger}(\xi, d, \boldsymbol{\alpha}, k) & :=\xi \mathcal{R}_{j}(d, \boldsymbol{\alpha}, k) \cap(2 \mathbb{Z}+1) \times \mathbb{Z} \quad(j \in\{1,3\}),
\end{aligned}
$$

et notons que

$$
\operatorname{vol}\left(\mathcal{R}_{j}(d, \boldsymbol{\alpha}, k)\right)=\frac{\operatorname{vol}\left(\mathcal{R}_{j}(d)\right)}{2^{k+2}} \quad(j \in\{1,3\}) .
$$

Comme dét $U_{\boldsymbol{\alpha}, \kappa}= \pm 2^{k+2}$, nous avons $\varrho_{T_{\alpha, k}}^{+}(d)=\varrho_{T}^{+}(d)(d \in 2 \mathbb{Z}+1)$.

Par ailleurs, nous avons, sous les hypothèses du Lemme 7.1,

$$
\delta_{\infty}\left(\mathcal{R}_{\boldsymbol{\alpha}, k}\right) \leqslant 2 \delta_{\infty}(\mathcal{R}), \quad \delta_{T_{\boldsymbol{\alpha}, k}}\left(\mathcal{R}_{\boldsymbol{\alpha}, k}\right) \leqslant \delta_{T}(\mathcal{R}) .
$$


Pour établir la première inégalité, rappelons la définition (7·19) de $\mathcal{R}_{\boldsymbol{\alpha}, k}$. Lorsque, par exemple $\boldsymbol{\alpha}=(1, \beta) \in \mathcal{W}_{k}$, la condition $U_{\boldsymbol{\alpha}, k} \boldsymbol{x} \in \mathcal{R}$ avec $\boldsymbol{x}=\left(x_{1}, x_{2}\right) \in \mathbb{R}^{2}$ implique $\left|\beta x_{1}+2^{k+2} x_{2}\right| \leqslant \delta_{\infty}(\mathcal{R})$, donc

$$
\left|x_{2}\right| \leqslant \delta_{\infty}(\mathcal{R}) / 2^{k+2}+\left|x_{1}\right| \leqslant \frac{5}{4} \delta_{\infty}(\mathcal{R})
$$

car $0 \leqslant \beta<2^{k+2}$. D'où

$$
\delta_{\infty}\left(\mathcal{R}_{\boldsymbol{\alpha}, k}\right)=\sup _{\boldsymbol{x} \in \mathcal{R}_{\boldsymbol{\alpha}, k}}\|\boldsymbol{x}\| \leqslant \sqrt{2} \sup _{\substack{\left|x_{1}\right| \leqslant \delta_{\infty}(\mathcal{R}) \\\left|\beta x_{1}+2^{k+2} x_{2}\right| \leqslant \delta_{\infty}(\mathcal{R})}} \max \left\{\left|x_{1}\right|,\left|x_{2}\right|\right\} \leqslant 2 \delta_{\infty}(\mathcal{R}) .
$$

La seconde inégalité de $(7 \cdot 32)$ résulte de l'identité

$$
\delta_{T_{\boldsymbol{\alpha}, k}}\left(\mathcal{R}_{\boldsymbol{\alpha}, k}\right)=\sup _{\boldsymbol{x} \in \mathcal{R}_{\boldsymbol{\alpha}, k}}\left|T_{\boldsymbol{\alpha}, k}(\boldsymbol{x})\right|^{1 / 4}=\sup _{\boldsymbol{x} \in \mathcal{R}}\left|T(\boldsymbol{x}) / 2^{k}\right|^{1 / 4}=\delta_{T}(\mathcal{R}) / 2^{k / 4} .
$$

Montrons que, pour $j=1$ ou 3 , la contribution à (7.30) des entiers $k \geqslant 3 \log _{2} \xi$ peut être englobée par le terme d'erreur de (7.26). Nous avons nécessairement $k \ll \log \xi$ et, pour chaque $k$, il y au plus, d'après $(7 \cdot 24)$, une quantité uniformément bornée de valeurs de $\boldsymbol{\alpha}$ à considérer. Lorsque $\xi / 2^{k} \leqslant \xi^{\varepsilon / 2}$, une majoration triviale fournit

$$
\sum_{\boldsymbol{x} \in \mathbb{Z}^{2} \cap \mathcal{R}_{\boldsymbol{\alpha}, k}(\xi)} \tau\left(T_{\boldsymbol{\alpha}, k}(\boldsymbol{x})\right) \ll(\sigma \xi)^{1+\varepsilon}
$$

ce qui induit, après report dans $(7 \cdot 30)$, un terme d'erreur acceptable dans $(7 \cdot 26)$.

Lorsque $\xi / 2^{k}>\xi^{\varepsilon / 2}$, nous sommes en mesure d'appliquer le théorème 4 de [21] pour évaluer la sommation relative à $m$ ou à $n$, selon que $\boldsymbol{\alpha}$ appartient à $\mathcal{W}_{k}$ ou $\mathcal{Z}_{k}$. Il vient

$$
\sum_{\boldsymbol{x} \in \mathbb{Z}^{2} \cap \mathcal{R}_{\boldsymbol{\alpha}, k}(\xi)} \tau\left(T_{\boldsymbol{\alpha}, k}(\boldsymbol{x})\right) \ll \frac{\|T\|^{\varepsilon / 2} \sigma^{2} \xi^{2}}{2^{k(1-\varepsilon)}} \exp \left\{\sum_{p \leqslant \xi} \frac{\varrho_{T}^{-}(p)}{p}\right\} \ll \frac{\|T\|^{\varepsilon} \sigma^{2} \xi^{2} \log \xi}{2^{k(1-\varepsilon)}} .
$$

En reportant dans $(7 \cdot 30)$, nous obtenons bien que la sous-somme correspondant à la condition $k \geqslant 3 \log _{2} \xi$ est

$$
\ll\|T\|^{\varepsilon} \sigma^{2} \xi^{2}(\log \xi)^{1-(1-\varepsilon) \log 8} \ll\|T\|^{\varepsilon} \sigma^{2} \xi^{2} / \sqrt{\log \xi}
$$

lorsque $\varepsilon$ est choisi suffisamment petit ; cette contribution est donc également négligeable au regard du terme d'erreur de $(7 \cdot 26)$.

Nous sommes à présent en mesure d'évaluer $Q_{1 j}(\xi ; T, \mathcal{R})$ lorsque $j=1$ ou 3 . D'après ce qui précède et le Lemme 5.2 , nous avons dans ce cas

$$
\begin{aligned}
\mathcal{Q}_{1 j}(\xi ; T, \mathcal{R})= & 4 \sum_{k \leqslant 3 \log _{2}} \sum_{\xi \boldsymbol{\alpha} \in \mathcal{W}_{k} \cup z_{k}} \sum_{d \leqslant y} \chi(d)\left|\Lambda\left(d ; T_{\boldsymbol{\alpha}, k}\right) \cap \mathcal{R}_{j}^{\dagger}(\xi, d, \boldsymbol{\alpha}, k)\right|+O\left(\frac{\|T\|^{\varepsilon} \sigma^{2} \xi^{2}}{\sqrt{\log \xi}}\right) \\
= & 4 \xi^{2} \sum_{d \leqslant y} \chi(d) \operatorname{vol}\left(\mathcal{R}_{j}(d)\right) \frac{\varrho_{T}^{+}(d)}{2 d^{2}} \sum_{k \leqslant 3 \log _{2} \xi} \frac{\left|\mathcal{W}_{k}\right|+\left|Z_{k}\right|}{2^{k+2}} \\
& +O\left((\sigma+\vartheta) \xi \sqrt{y}(\log \xi)^{\varepsilon / 2} \sum_{k \leqslant 3 \log _{2}} \sum_{\xi \boldsymbol{\alpha} \in \mathcal{W}_{k} \cup z_{k}}\left\|T_{\boldsymbol{\alpha}, k}\right\|^{\varepsilon / 20}+\frac{\|T\|^{\varepsilon} \sigma^{2} \xi^{2}}{\sqrt{\log \xi}}\right) .
\end{aligned}
$$

Puisque $\left\|T_{\boldsymbol{\alpha}, k}\right\| \ll 8^{k}\|T\|$ d'après (7·17) et comme $\left|\mathcal{W}_{k} \cup \mathcal{Z}_{k}\right| \ll_{J} 1$ par (7.24), nous pouvons écrire

$$
\sum_{k \leqslant 3 \log _{2} \xi} \sum_{\boldsymbol{\alpha} \in \mathcal{W}_{k} \cup z_{k}}\left\|T_{\boldsymbol{\alpha}, k}\right\|^{\varepsilon / 20} \ll\|T\|^{\varepsilon / 20} \sum_{k \leqslant 3 \log _{2} \xi} 8^{k \varepsilon / 20} \ll_{J}\|T\|^{\varepsilon}(\log \xi)^{\varepsilon / 2} .
$$


Il vient, compte tenu de $(7 \cdot 22)$

$$
\begin{aligned}
\mathcal{Q}_{j}(\xi ; T, \mathcal{R})= & \frac{3}{2} K_{2}(T) \xi^{2} \sum_{d \leqslant y} \chi(d) \operatorname{vol}\left(\mathcal{R}_{j}(d)\right) \frac{\varrho_{T}^{+}(d)}{d^{2}} \\
& +O\left(\|T\|^{\varepsilon}(\sigma+\vartheta) \xi(\log \xi)^{\varepsilon} \sqrt{y}+\frac{\|T\|^{\varepsilon} \sigma^{2} \xi^{2}}{\sqrt{\log \xi}}\right) .
\end{aligned}
$$

Rappelons que

$$
\mathcal{R}_{1}(d)=\mathcal{R}, \quad \mathcal{R}_{3}(d) \backslash \mathcal{R}=\left\{\boldsymbol{x} \in \mathcal{R}: T(\boldsymbol{x}) y / \vartheta^{4} \leqslant d\right\} .
$$

En notant que, d'après $(3 \cdot 11)$,

$$
\sum_{d>z} \chi(d) \frac{\varrho_{T}^{+}(d)}{d^{2}} \ll_{\varepsilon} \frac{\|T\|^{\varepsilon}}{\log z} \quad(z \geqslant 2),
$$

il s'ensuit que, pour $j=1$ ou 3 ,

$$
\begin{gathered}
\sum_{d \leqslant y} \chi(d) \operatorname{vol}\left(\mathcal{R}_{j}(d)\right) \frac{\varrho_{T}^{+}(d)}{d^{2}}=\operatorname{vol}(\mathcal{R}) \sum_{d \leqslant y} \chi(d) \frac{\varrho_{T}^{+}(d)}{d^{2}}+O\left(\int_{\mathcal{R}} \sum_{T(\boldsymbol{x}) y / \vartheta^{4}<d \leqslant y} \chi(d) \frac{\varrho_{T}^{+}(d)}{d^{2}} \mathrm{~d} \boldsymbol{x}\right) \\
=\operatorname{vol}(\mathcal{R}) \sum_{d \geqslant 1} \chi(d) \frac{\varrho_{T}^{+}(d)}{d^{2}}+O\left(\frac{\|T\|^{\varepsilon} \sigma^{2}}{\log \xi}\right)=\frac{\operatorname{vol}(\mathcal{R}) \pi K(T)}{4 K_{2}(T)}+O\left(\frac{\|T\|^{\varepsilon} \sigma^{2}}{\log \xi}\right),
\end{gathered}
$$

où nous avons estimé le terme d'erreur grâce à (7·35) et à la majoration

$$
\int_{\mathcal{R}} \frac{\mathrm{d} x}{\log \left(T(\boldsymbol{x}) y / \vartheta^{4}+2\right)} \leqslant \frac{\sigma^{2}}{\operatorname{dét~} M} \int_{\|\boldsymbol{x}\| \leqslant 1} \frac{\mathrm{d} x}{\log (J(\boldsymbol{x}) Y+2)} \ll \frac{\sigma^{2}}{\{\log (2+Y)\} \text { dét } M},
$$

où l'on a posé $Y=\sigma^{4} y /\left(\vartheta^{4} q\right)$ : nous avons $Y \gg \sigma^{2} \xi^{2} /(\sqrt{\|T\|} \log \xi)$ par (7.27) et $\|T\| \leqslant(\log \xi)^{1 / \varepsilon}$, nous obtenons la majoration annoncée.

En reportant dans $(7 \cdot 34)$ puis dans $(7 \cdot 29)$, nous obtenons

$$
\begin{aligned}
\mathcal{Q}_{1}(\xi ; T, \mathcal{R})= & \frac{3}{4} \pi K(T) \operatorname{vol}(\mathcal{R}) \xi^{2}+\mathcal{Q}_{12}(\xi ; T, \mathcal{R}) \\
& +O\left(\|T\|^{\varepsilon}(\sigma+\vartheta) \xi \sqrt{y}(\log \xi)^{\varepsilon}+\frac{\|T\|^{\varepsilon} \sigma^{2} \xi^{2}}{\sqrt{\log \xi}}\right) .
\end{aligned}
$$

Majorons à présent $\mathcal{Q}_{12}(\xi ; T, \mathcal{R})$. Posons

$$
\Upsilon(\Xi, b):=\sum_{\substack{x \in \mathcal{R}(\Xi) \cap \mathbb{Z}^{2} \\(m, n)=1}} \sum_{\substack{d \mid b^{4} T(x) \\ y<d \leqslant \vartheta^{4} \xi^{4} / y}} \chi(d),
$$

et notons la majoration triviale

$$
\Upsilon(\Xi, b) \ll \tau\left(b^{4}\right) \sum_{x \in \mathcal{R}(\Xi) \cap \mathbb{Z}^{2}} \tau(T(\boldsymbol{x})) \ll\|T\|^{\varepsilon} \tau\left(b^{4}\right) \sigma^{2} \Xi^{2} \log \Xi
$$

qui résulte du Théorème 4.1. Nous avons, par (7·31) avec $j=2$,

$$
\mathcal{Q}_{12}(\xi ; T, \mathcal{R})=4 \sum_{\substack{b \geqslant 1 \\ 2 \nmid b}} \Upsilon(\xi / b, b)=4 \sum_{\substack{1 \leqslant b \leqslant L \\ 2 \nmid b}} \Upsilon(\xi / b, b)+O\left(\frac{\|T\|^{\varepsilon} \sigma^{2} \xi^{2}}{\sqrt{\log \xi}}\right),
$$

où l'on a posé $L:=(\log \xi)^{20}$. 
Estimons à présent $\Upsilon(\Xi, b)$ lorsque $\xi / L \leqslant \Xi \leqslant \xi$ et $b \leqslant L$. Pour obtenir un résultat satisfaisant, il est essentiel de traiter non trivialement la somme intérieure de $(7 \cdot 37)$ : nous exploitons le fait qu'elle est

$$
\ll \Delta\left(b^{4} T(\boldsymbol{x}), \chi\right) \log \left(2 \vartheta^{2} \xi^{2} / y\right) \ll \tau\left(b^{4}\right) \Delta(T(\boldsymbol{x}, \chi)) \log _{2} \xi,
$$

compte tenu de (7·27). Dans l'idée d'appliquer l'inégalité de Cauchy-Schwarz, nous observons qu'en vertu du Théorème 4.1, du Lemme 4.3, et du Théorème 4.4, nous pouvons écrire

$$
\sum_{\substack{x \in \mathcal{R}(\Xi) \cap \mathbb{Z}^{2} \\(m, n)=1}} \Delta(T(\boldsymbol{x}), \chi)^{2} \ll\|T\|^{\varepsilon} \sigma^{2} \Xi^{2} E_{J}(\sigma \Xi ; \Delta(\cdot, \chi)) \ll\|T\|^{\varepsilon} \sigma^{2} \Xi^{2} \mathcal{L}(\Xi)^{O(1)} .
$$

Posons

$$
B\left(\Xi, y_{1}, y_{2}\right):=\sum_{\substack{x \in \mathcal{R}(\Xi) \cap \mathbb{Z}^{2} \\(m, n)=1 \\ \exists d \mid T(\boldsymbol{x}): y_{1}<d \leqslant y_{2}}} 1 \quad\left(\left(\Xi, y_{1}, y_{2}\right) \in \mathbb{R}^{+3}\right)
$$

Compte tenu de ce qui précède, nous pouvons écrire

$$
\Upsilon(\Xi, b)^{2} \ll\|T\|^{\varepsilon} \tau\left(b^{4}\right)^{2}\left(\log _{2} \xi\right)^{2} B\left(\Xi, y / b^{4}, \vartheta^{4} \xi^{4} / y\right) \sigma^{2} \Xi^{2} \mathcal{L}(\Xi)^{O(1)} .
$$

Admettons provisoirement la validité de l'estimation

$$
B\left(\Xi, y_{1}, y_{2}\right) \ll\|T\|^{\varepsilon}(\sigma+\vartheta)^{2} \Xi^{2}\left(\frac{\vartheta^{2} \Xi^{2} / y_{1}+y_{2} / \vartheta^{2} \Xi^{2}}{(\log \xi)^{Q(2 \eta)}}+\frac{\log \left\{(\log \xi) y_{2} / y_{1}\right\}}{(\log \xi)^{Q(1+\eta)}}\right)
$$

lorsque $\Xi, y_{1}, y_{2}$ satisfont $\xi / L \leqslant \Xi \leqslant \xi, 1 \leqslant y_{1} \leqslant y_{2}$, et $0<\eta<\frac{1}{2}$.

En reportant dans $(7 \cdot 40)$ puis $(7 \cdot 38)$, nous obtenons

$$
\mathcal{Q}_{12}(\xi ; T, \mathcal{R}) \ll\|T\|^{\varepsilon}(\sigma+\vartheta) \sigma \xi^{2}\left(\frac{\vartheta \xi}{(\log \xi)^{2 \delta} \sqrt{y}}+\frac{1}{(\log \xi)^{\delta}}\right) .
$$

dès que

$$
\delta<\max _{\eta \in] 0,1 / 2[} \min \left\{\frac{1}{4} Q(2 \eta), \frac{1}{2} Q(1+\eta)\right\} .
$$

Nous pouvons choisir $\delta=0,021>\frac{1}{48}$, correspondant à la valeur $\eta=0,305$. En spécialisant alors $y:=\vartheta^{2} \xi^{2} /(\log \xi)^{2 \delta}$ dans $(7 \cdot 36)$ nous obtenons bien $(7 \cdot 5)$.

\subsection{Complétion de l'argument}

Nous prouvons ici la majoration $(7 \cdot 41)$. Le résultat étant trivialement acquis si l'on a $y_{1} \leqslant \vartheta^{2} \Xi^{2} /(\log \xi)^{Q(2 \eta)}$ ou $y_{2} \geqslant \vartheta^{2} \Xi^{2}(\log \xi)^{Q(2 \eta)}$, nous pouvons supposer dans toute la suite que $\log \left(y_{2} / y_{1}\right) \ll \log _{2} \xi$.

Rappelons la définition de la constante $c_{0}$ dans $(4 \cdot 2)$. Soit $\left.\delta=\delta(\kappa) \in\right] 0,1[$ tel que $\|T\|^{\delta} \leqslant c_{0} \sigma \xi / L$. Supposons alors, sans perte de généralité, que $\varepsilon \leqslant \delta / 4000$, fixons $z=z(\varepsilon)>3$ tel que $(\log 2) / \log z<\varepsilon$ et introduisons la fonction arithmétique additive

$$
\Omega_{z}(n):=\sum_{\substack{p^{\nu} \| n \\ p>z}} \nu
$$

Ainsi toute fonction arithmétique $F$ sous-multiplicative satisfaisant à $0 \leqslant F \ll 2^{\Omega_{z}}$ vérifie les hypothèses du Théorème 4.1 pour la forme $T$. 
Soit $\eta \in] 0, \frac{1}{2}\left[\right.$. Désignons par $B_{j}(1 \leqslant j \leqslant 3)$ les contributions respectives à $(7 \cdot 39)$ des vecteurs $\boldsymbol{x}$ satisfaisant respectivement aux conditions supplémentaires

$$
\begin{array}{ll}
\left(B_{1}\right) & \Omega_{z}(T(\boldsymbol{x}))>(1+\eta) \log _{2} \xi, \\
\left(B_{2}\right) & \Omega_{z}(T(\boldsymbol{x})) \leqslant(1+\eta) \log _{2} \xi, T(\boldsymbol{x})>\vartheta^{4} \Xi^{4} /(\log \xi)^{2}, \\
\left(B_{3}\right) & T(\boldsymbol{x}) \leqslant \vartheta^{4} \Xi^{4} /(\log \xi)^{2},
\end{array}
$$

de sorte que $B\left(\Xi, y_{1}, y_{2}\right) \leqslant \sum_{1 \leqslant j \leqslant 3} B_{j}$.

Nous commençons par observer que, par $(5 \cdot 24)$ avec $\beta:=1 /(\log \xi)^{2}$, nous avons

$$
B_{3} \ll\|T\|^{\varepsilon} \vartheta^{2} \Xi^{2} / \sqrt{\log \xi}
$$

La méthode paramétrique fournit lorsque $y \in[1,2[$

$$
B_{1} \leqslant \sum_{\substack{\boldsymbol{x} \in \mathbb{Z}^{2} \\\|\boldsymbol{x}\| \leqslant \sigma \Xi}} y^{\Omega_{z}(T(\boldsymbol{x}))-(1+\eta) \log _{2} \xi}
$$

En vertu du Théorème 4.1, il suit

$$
B_{1} \leqslant \frac{\|T\|^{\varepsilon} \sigma^{2} \Xi^{2}}{(\log \xi)^{(1+\eta) \log y}} \prod_{p \leqslant \sigma \xi}\left\{1+(y-1) \frac{\varrho_{T}^{-}(p)}{p}\right\} \ll \frac{\|T\|^{2 \varepsilon} \sigma^{2} \Xi^{2}}{(\log \xi)^{(1+\eta) \log y-y+1}} \ll \frac{\|T\|^{2 \varepsilon} \sigma^{2} \Xi^{2}}{(\log \xi)^{Q(1+\eta)}}
$$

pour le choix $y:=1+\eta$.

Majorons $B_{2}$. Par hypothèse, chaque vecteur $\boldsymbol{x}$ de $\mathbb{Z}^{2}$ compté dans la somme est tel que $T(\boldsymbol{x})$ possède un diviseur $d$ dans $\left.] y_{1}, y_{2}\right]$. L'une des quatre conditions suivantes est alors nécessairement vérifiée :

$$
\begin{aligned}
& \text { (i) } \Omega_{z}(d) \leqslant(1-\eta) \log _{2} \xi \text { et } y_{1}<d \leqslant \vartheta^{2} \Xi^{2} ; \\
& \text { (ii) } \Omega_{z}(d) \leqslant 2 \eta \log _{2} \xi \text { et } \vartheta^{2} \Xi^{2}<d \leqslant y_{2} ; \\
& \text { (iii) } \Omega_{z}(d)>2 \eta \log _{2} \xi \text { et } \vartheta^{2} \Xi^{2}<d \leqslant y_{2} ; \\
& \text { (iv) } \Omega_{z}(d)>(1-\eta) \log _{2} \xi \text { et } y_{1}<d \leqslant \vartheta^{2} \Xi^{2} .
\end{aligned}
$$

Notons $B_{2 j}(1 \leqslant j \leqslant 4)$ les contributions correspondantes à la somme $B_{2}$. D'après le Lemme 5.1 et le Théorème 4.1 , nous avons pour tout $y \leqslant 1$

$$
\begin{aligned}
B_{21} & \ll \sum_{y_{1}<d \leqslant \vartheta^{2} \Xi^{2}} y^{\Omega_{z}(d)-(1-\eta) \log _{2} \xi} \sum_{\substack{x \in \mathcal{R}(\Xi) \cap \Lambda(d ; T) \\
(m, n)=1}} 1 \\
& \ll(\log \xi)^{-(1-\eta) \log y} \sum_{y_{1}<d \leqslant \vartheta^{2} \Xi^{2}} y^{\Omega_{z}(d)} \frac{\varrho_{T}^{+}(d)}{d}\left\{\frac{\sigma^{2} \Xi^{2}}{d}+1\right\} \\
& \ll\left(\sigma^{2}+\vartheta^{2}\right)(\log \xi)^{-(1-\eta) \log y} \sum_{y_{1}<d \leqslant \vartheta^{2} \Xi^{2}} y^{\Omega_{z}(d)} \frac{\varrho_{T}^{+}(d) \Xi^{2}}{d^{2}} \\
& \ll\|T\|^{\varepsilon}\left(\sigma^{2}+\vartheta^{2}\right) \Xi^{2} \log \left(2 \vartheta^{2} \Xi^{2} / y_{1}\right)(\log \xi)^{y-1-(1-\eta) \log y} \\
& \ll\|T\|^{\varepsilon}\left(\sigma^{2}+\vartheta^{2}\right) \Xi^{2}(\log \xi)^{-Q(1-\eta)} \log \left(2 \vartheta^{2} \Xi^{2} / y_{1}\right)
\end{aligned}
$$

pour le choix $y:=1-\eta$. 
D'après le Lemme 5.1, nous avons encore, pour tout $y \leqslant 1$,

$$
\begin{aligned}
B_{22} & \ll \sum_{\vartheta^{2} \Xi^{2}<d \leqslant y_{2}} y^{\Omega_{z}(d)-2 \eta \log _{2} \xi} \sum_{\substack{x \in \mathcal{R}(\Xi) \cap \Lambda(d) \\
(m, n)=1}} 1 \\
& \ll(\log \xi)^{-2 \eta \log y} \sum_{\vartheta^{2} \Xi^{2}<d \leqslant y_{2}} y^{\Omega_{z}(d)} \frac{\varrho_{T}^{+}(d)}{d}\left\{\frac{\sigma^{2} \Xi^{2}}{d}+1\right\} \\
& \ll \frac{1+\sigma^{2} / \vartheta^{2}}{(\log \xi)^{2 \eta \log y}} \sum_{(\vartheta \Xi)^{2}<d \leqslant y_{2}} y^{\Omega_{z}(d)} \frac{\varrho_{T}^{+}(d)}{d} \\
& \ll\|T\|^{\varepsilon}\left\{1+\sigma^{2} / \vartheta^{2}\right\}(\log \xi)^{y-1-2 \eta \log y} y_{2} \ll\|T\|^{\varepsilon}\left\{1+\sigma^{2} / \vartheta^{2}\right\} y_{2} /(\log \xi)^{Q(2 \eta)}
\end{aligned}
$$

pour le choix $y=2 \eta$.

Pour estimer $B_{23}$ et $B_{24}$, nous raisonnons sur le diviseur complémentaire $\ell=T(\boldsymbol{x}) / d$. Nous avons $\ell \leqslant \vartheta^{4} \Xi^{4} / d$ et

$$
\Omega_{z}(\ell)=\Omega_{z}(T(\boldsymbol{x}))-\Omega_{z}(d) \leqslant(1+\eta) \log _{2} \xi-\Omega_{z}(d) .
$$

Ainsi, dans $B_{23}, \ell$ joue le rôle de $d$ dans $B_{21}$ et $\vartheta^{4} \Xi^{4} / y_{2}(\log \xi)^{2}$ celui de $y_{1}$; similairement, dans $B_{24}, \ell$ joue le rôle de $d$ dans $B_{22}$ et $\vartheta^{4} \Xi^{4} / y_{1}$ celui de $y_{2}$.

Comme $Q(1+\eta) \leqslant Q(1-\eta)$, nous obtenons bien ainsi $(7 \cdot 41)$.

Cela achève la démonstration du Lemme 7.1.

\section{Preuve du Théorème $\mathbf{1 . 1}$}

\section{1. Estimation asymptotique de $N_{P}(B)$}

Nous allons établir ici l'existence d'une constante $C_{P}$, indépendante de $H$, telle que l'on ait

$$
N_{P}(B)=\left\{C_{P} \operatorname{vol}\left(\mathcal{R}_{P}\right)+O\left(\frac{1}{(\log B)^{1 / 100}}\right)\right\} B \log B \quad(B \rightarrow \infty)
$$

Nous faisons appel à la Proposition 2.1, dont la formule (2.6) permet de réduire l'estimation de $N_{P}(B)$ à celle des quantités $S_{P}(\xi, d)$, définies en $(2 \cdot 5)$, et qui sont ellesmêmes exprimables, via $(2 \cdot 7)$, en fonction des sommes $\mathfrak{S}(\xi, d ; T)$ définies en $(2 \cdot 3)$.

Commençons donc par évaluer les quantités $\mathfrak{S}(\xi, d ; T)$. Plaçons-nous d'abord dans l'hypothèse où le contenu de $T$ est premier avec $d$. Rappelons les notations $(2 \cdot 8)$ et $(5 \cdot 2)$ et posons

$$
\mathscr{G}_{d}(\xi, \mathcal{A} ; e, b):=\sum_{x \in e \mathcal{A}_{d_{b} / e} \cap \mathcal{R}_{T}(\xi / b)} r\left(\frac{T_{b}(\boldsymbol{x})}{d_{b}}\right) \quad\left(\xi \geqslant 0, \mathcal{A} \in \mathcal{U}_{T}(d), b \in \mathbb{N}^{*}, e \mid d_{b}\right) .
$$

La décomposition canonique $(5 \cdot 8)$ de l'ensemble $\Lambda(d ; T)$ nous permet d'écrire

$$
\begin{aligned}
\mathfrak{S}(\xi, d ; T) & =\sum_{b \mid \psi(d)} \sum_{x \in \Lambda^{*}\left(d_{b} ; T_{b}\right) \cap \mathcal{R}_{T}(\xi / b)} r\left(\frac{T_{b}(\boldsymbol{x})}{d_{b}}\right) \\
& =\sum_{b \mid \psi(d)} \sum_{\mathcal{A} \in \mathcal{U}_{T}\left(d_{b}\right)} \sum_{e \mid d_{b}} \mu(e) \mathscr{G}_{d}(\xi, \mathcal{A} ; e, b) .
\end{aligned}
$$

Nous sommes ainsi ramenés à un comptage de points entiers sur un réseau de déterminant $d_{b} e$. 
L'estimation de $\mathscr{G}(\xi, \mathcal{A}, e)$ relève du Lemme 7.1. Pour nous placer dans les hypothèses de cet énoncé, nous nous donnons une base minimale $\left(\boldsymbol{e}_{1}, \boldsymbol{e}_{2}\right)$ de vecteurs du réseau $e \mathcal{A}_{d_{b} / e}$ (ainsi $\left\|\boldsymbol{e}_{1}\right\|$ et $\left\|\boldsymbol{e}_{2}\right\|$ sont les minimums successifs des normes d'éléments de $e \mathcal{A}_{d_{b} / e}$ ) et nous désignons par $E$ la matrice de $\mathcal{M}_{2}(\mathbb{Z})^{*}$ qui transforme la base canonique en $\left(\boldsymbol{e}_{1}, \boldsymbol{e}_{2}\right)$, de sorte que

$$
e \mathcal{A}_{d_{b} / e}=E\left(\mathbb{Z}^{2}\right) .
$$

Posant $\mathcal{R}_{E}^{\star}:=\left\{\boldsymbol{x} \in \mathbb{R}^{2}: E \boldsymbol{x} \in \mathcal{R}_{T} / b\right\}$, nous avons donc

$$
\operatorname{vol}\left(\mathcal{R}_{E}^{\star}\right)=\frac{\operatorname{vol}\left(\mathcal{R}_{T}\right)}{b^{2} \operatorname{dét} E}=\frac{\operatorname{vol}\left(\mathcal{R}_{T}\right)}{b^{2} d_{b} e} .
$$

Introduisant la forme binaire définie par $M_{E}(\boldsymbol{v})=T_{b}(E \boldsymbol{v}) / d_{b}\left(\boldsymbol{v} \in \mathbb{Z}^{2}\right)$, nous pouvons écrire

$$
\mathscr{G}_{d}(\xi, \mathcal{A} ; e, b)=\sum_{\boldsymbol{v} \in \mathbb{Z}^{2} \cap \mathcal{R}_{E}^{\star}(\xi)} r\left(M_{E}(\boldsymbol{v})\right)=\mathfrak{S}\left(\xi ; M_{E}, \mathcal{R}_{E}^{\star}\right),
$$

avec la notation $(7 \cdot 2)$.

Estimons ensuite dans le présent contexte les quantités intervenant dans le terme d'erreur de $(7 \cdot 5)$. Nous avons d'abord

$$
\begin{aligned}
\delta_{M_{E}}\left(\mathcal{R}_{E}^{\star}\right) & =\sup _{\boldsymbol{v} \in \mathcal{R}_{E}^{\star}}\left\{\left|M_{E}(\boldsymbol{v})\right|^{1 / 4}\right\}=\sup _{\boldsymbol{x} \in \mathcal{R}_{T} / b}\left\{\left|T_{b}(\boldsymbol{x}) / d_{b}\right|^{1 / 4}\right\} \\
& =\sup _{\boldsymbol{x} \in \mathcal{R}_{T}}\left\{|T(\boldsymbol{x}) / d|^{1 / 4}\right\}=\frac{\delta_{T}\left(\mathcal{R}_{T}\right)}{d^{1 / 4}}=: \vartheta_{d} .
\end{aligned}
$$

Puis

$$
\left\|M_{E}\right\| \ll\|T\|\|E\|^{4} / d_{b} \ll\|T\| d_{b}^{4} e^{3} \leqslant\|T\| d^{7} .
$$

Enfin

$$
\delta_{\infty}\left(\mathcal{R}_{E}^{\star}\right)=\sup _{\boldsymbol{v} \in \mathcal{R}_{E}^{\star}}\|\boldsymbol{v}\| \ll \frac{\delta_{\infty}\left(\mathcal{R}_{T}\right)}{b} .
$$

Notant $\sigma_{T}:=\delta_{\infty}\left(\mathcal{R}_{T}\right)$, nous déduisons donc du Lemme 7.1 que

$$
\mathscr{G}_{d}(\xi, \mathcal{A} ; e, b)=\frac{\pi K_{E} \operatorname{vol}\left(\mathcal{R}_{T}\right) \xi^{2}}{b^{2} d_{b} e}+O_{T}\left(\frac{\left(\sigma_{T} / b+\vartheta_{d}\right)^{2} d^{\varepsilon} \xi^{2}}{(\log \xi)^{1 / 48}}\right),
$$

où $K_{E}:=K\left(M_{E}\right)=\prod_{p} K_{p}\left(M_{E}\right)$ est défini par les formules $(7 \cdot 6)$ avec $T=M_{E}$.

Reportons (8.3) dans (8.2). La constante du terme principal est donc un multiple de

$$
W(d ; T):=\sum_{b \mid \psi(d)} \sum_{\mathcal{A} \in \mathcal{U}_{T}\left(d_{b}\right)} \sum_{e \mid d_{b}} \frac{K_{E} \mu(e)}{b^{2} d_{b} e} .
$$

On peut calculer la valeur de cette expression par des manipulations identiques à celles qui apparaissent dans la preuve du théorème 3 de [3] et dont nous omettons ici les détails. Nous obtenons

$$
W(d ; T):=\prod_{p} W_{p}(d ; T)
$$

avec

$$
\begin{aligned}
& W_{2}(d ; T):=K_{2}(T), \\
& W_{p}(d ; T):=\left(1-\frac{\chi(p)}{p}\right) \sum_{\nu \geqslant 0} \frac{\chi\left(p^{\nu}\right) \varrho_{T}^{+}\left(p^{\nu+v_{p}(d)}\right)}{p^{2 \nu+2 v_{p}(d)}} \quad(p>2) .
\end{aligned}
$$


De plus, nous pouvons déduire de $(7 \cdot 7)$ que, pour tout nombre premier $p$, nous avons

$$
W_{p}(d ; T)=\lim _{\nu \rightarrow \infty} \frac{1}{p^{3 \nu+v_{p}(d)}} \sum_{\substack{(\boldsymbol{x}, s, t) \in\left(\mathbb{Z} / p^{\nu} \mathbb{Z}\right)^{4} \\ T(x) \equiv p^{v_{p}(d)}\left(s^{2}+t^{2}\right)\left(\bmod p^{\nu}\right)}} 1 .
$$

Pour estimer la contribution du terme d'erreur de (8·3) à la somme (8·2), nous observons d'abord que le nombre de termes apparaissant dans la somme (8.2) vaut

$$
n(d ; T):=\sum_{b \mid \psi(d)} \sum_{\mathcal{A} \in \mathcal{U}_{P}\left(d_{b}\right)} \sum_{e \mid d_{b}} \mu(e)^{2}=\sum_{b \mid \psi(d)} \frac{\varrho_{T}^{*}\left(d_{b}\right)}{\varphi\left(d_{b}\right)} 2^{\omega\left(d_{b}\right)} \ll_{T, \varepsilon} d^{\varepsilon} .
$$

Il suit finalement

$$
\mathfrak{S}(\xi, d ; T)=\pi W(d ; T) \operatorname{vol}\left(\mathcal{R}_{T}\right) \xi^{2}+O_{T, \varepsilon}\left(\frac{d^{\varepsilon}\left(\sigma_{T}^{2}+\vartheta_{T}^{2}\right) \xi^{2}}{(\log \xi)^{1 / 48}}\right),
$$

où nous avons posé $\vartheta_{T}:=\delta_{T}\left(\mathcal{R}_{T}\right)$.

Montrons que (8.7) persiste lorsque $d$ n'est pas premier avec le contenu $c(T)$ de la forme $T$. À cette fin, posons $m:=(d, c(T))$ et observons que $\mathfrak{S}(\xi, d ; T)=\mathfrak{S}(\xi, d / m ; T / m)$. Comme $W(d ; T)=W(d / m ; T / m)$ et $\operatorname{vol}\left(\mathcal{R}_{T}\right)=\operatorname{vol}\left(\mathcal{R}_{T / m}\right)$, nous obtenons bien l'extension annoncée.

Nous devons ensuite appliquer $(2 \cdot 7)$. À cette fin, il est nécessaire de disposer d'une majoration de $W(d ; T)$. Le Lemme 3.2 permet d'écrire

$$
\begin{aligned}
& W_{p}(d ; T) \ll p^{-\left\lceil v_{p}(d) / 4\right\rceil} \quad(p \nmid c(T)) \\
& W_{p}(d ; T) \ll v_{p}(c(T)) \quad(p \mid c(T)) \\
& W_{p}(d ; T)=1-\frac{\chi(p)}{p}+\sum_{1 \leqslant \nu \leqslant 4} \chi\left(p^{\nu}\right) \frac{\varrho_{T}^{+}\left(p^{\nu}\right)}{p^{2 \nu}}+O\left(\frac{1}{p^{2}}\right) \quad(p \nmid d c(T)) \\
& W_{p}(d ; T)=1+\chi(p) \frac{\varrho_{T}^{-}(p)-1}{p}+O\left(\frac{1}{p^{2}}\right) \quad\left(p \nmid d c_{0} \mathcal{D}(T)\right),
\end{aligned}
$$

où la première majoration fait usage de l'identité $\nu-\lfloor 3 \nu / 4\rfloor=\lceil\nu / 4\rceil$. Cela implique, lorsque $d$ est sans facteur carré et pour tout $\varepsilon>0$,

$$
W(d ; T) \ll_{\varepsilon}\|T\|^{\varepsilon}(d, c(T))^{1-\varepsilon} / d^{1-\varepsilon} \ll_{T, \varepsilon} 1 / d^{1-\varepsilon} .
$$

Rappelons alors les définitions (2.8) et (8.6) et reportons l'estimation (8.8) dans la formule $(2 \cdot 7)$ en tenant compte de la majoration

$$
\left\|T_{\ell}\right\| \ll\|T\| \ell^{4} .
$$

Nous obtenons, lorsque la forme binaire $P$ est fixée,

$$
S_{P}(\xi, d)=\pi W^{*}(d ; P) \operatorname{vol}\left(\mathcal{R}_{P}\right) \xi^{2}+O\left(\frac{d^{\varepsilon} \xi^{2}}{(\log \xi)^{1 / 48}}\right),
$$

avec

$$
W^{*}(d ; P):=\sum_{\ell \in \mathbb{N}^{*}} \frac{\mu(\ell) W\left(d_{\ell} ; P_{\ell}\right)}{\ell^{2}}
$$


Rappelons la définition des ensembles $\mathcal{E}_{\nu}$ en $(7 \cdot 3)$. La relation $(8 \cdot 4)$ permet d'écrire la formule

$$
W\left(d_{\ell} ; P_{\ell}\right)=\prod_{p} W_{p}\left(d_{\ell} ; P_{\ell}\right)
$$

où, d'après $(8.5)$, chaque terme $W_{p}\left(d_{\ell} ; P_{\ell}\right)$ dépend uniquement de $v_{p}(\ell)$. La quantité $W^{*}(d ; P)$ admet donc une réprésentation sous la forme d'un produit eulérien, soit

$$
W^{*}(d ; P)=\prod_{p} W_{p}^{*}(d ; P),
$$

avec

$$
\begin{aligned}
W_{p}(d ; P) & :=W_{p}^{*}(d ; P)-W_{p}\left(d_{p} ; P_{p}\right) / p^{2} \\
& =\lim _{\nu \rightarrow \infty} \frac{1}{p^{3 \nu+v_{p}(d)}} \sum_{\substack{(x, s, t) \in\left(\mathbb{Z} / p^{\nu} \mathbb{Z}\right)^{4} \\
P(\boldsymbol{x}) \equiv p^{v}(d)\left(s^{2}+t^{2}\right)\left(\bmod p^{\nu}\right) \\
p \nmid(m, n)}} 1 \quad(p>2),
\end{aligned}
$$

et

$$
W_{2}^{*}(d ; P)=W_{2}^{*}(1 ; P)=\lim _{n \rightarrow \infty} \frac{1}{2^{2 \nu-1}} \sum_{\substack{x \in\left(\mathbb{Z} / 2^{\nu} \mathbb{Z}\right)^{2} \\ P(\boldsymbol{x}) \in \mathcal{E}_{\nu} \\ 2 \nmid(m, n)}} 1
$$

puisque

$$
\left|\left\{(s, t) \in\left(\mathbb{Z} / 2^{\nu} \mathbb{Z}\right)^{2}: s^{2}+t^{2} \equiv a\left(\bmod 2^{\nu}\right)\right\}\right|=2^{\nu+1}
$$

lorsque $a \in \mathcal{E}_{\nu}$.

L'expression (8.11) implique également

$$
W_{p}^{*}(d ; P):=\left\{\begin{array}{l}
\left(1-\frac{\chi(p)}{p}\right) \sum_{\nu \geqslant 0} \frac{\chi\left(p^{\nu}\right) \varrho_{P}^{*}\left(p^{\nu+v_{p}(d)}\right)}{p^{2 \nu+2 v_{p}(d)}} \quad\left(p>2, v_{p}(d)=1\right), \\
\left(1-\frac{\chi(p)}{p}\right)\left\{1-\frac{1}{p^{2}}+\sum_{\nu \geqslant 1} \frac{\chi\left(p^{\nu}\right) \varrho_{P}^{*}\left(p^{\nu}\right)}{p^{2 \nu}}\right\} \quad\left(p>2, v_{p}(d)=0\right),
\end{array}\right.
$$

Enfin, nous observons que (8.8) implique trivialement

$$
W^{*}(d ; P) \ll 1 / d^{1-\varepsilon} \quad\left(\mu(d)^{2}=1\right) .
$$

Reportons à présent (8.9) dans la formule $(2 \cdot 6)$ pour $N_{P}(B)$ en nous donnant un paramètre de troncature $D$. La contribution des entiers $d>D$, traitée comme un terme d'erreur, est majorée grâce à (6·2). Nous obtenons

$$
\begin{aligned}
& \ll \sum_{d>D} \mu(d)^{2} \sum_{n \leqslant B \sqrt{\|P\| / d^{3 / 2}}}\left|f_{d}(n)\right|\left(\frac{B}{d^{2-\varepsilon} n}+\frac{B^{1 / 2+\varepsilon}}{d^{1 / 2+\varepsilon} n^{1 / 2+\varepsilon}}\right) \\
& \ll B(\log B) \sum_{d>D} \frac{\mu(d)^{2} r(d)}{d^{2-\varepsilon}}+B \sum_{d>D} \frac{\mu(d)^{2} r(d)}{d^{5 / 4-\varepsilon / 2}} \\
& \ll \frac{B \log B}{D^{1-\varepsilon}}+\frac{B}{D^{1 / 4-\varepsilon / 2}} \ll \frac{B \log B}{D^{1-\varepsilon / 2}},
\end{aligned}
$$


quitte à choisir $\varepsilon$ assez petit et $D \leqslant \sqrt{\log B}$.

Évaluons ensuite le terme principal, correspondant donc au domaine de sommation $d \leqslant D$. La contribution du terme d'erreur de (8.9) est

$$
\ll B(\log B)^{47 / 48} D^{1+8 \varepsilon} .
$$

le choix $D:=(\log B)^{1 / 96}$ est donc acceptable au regard du résultat annoncé (8.1). La contribution du terme principal peut enfin être évaluée grâce au Lemme 2.2. Elle vaut

$$
\begin{aligned}
\frac{1}{8} B \pi \operatorname{vol}\left(\mathcal{R}_{P}\right) & \sum_{d \leqslant D} \frac{\mu(d) \chi(d) W^{*}(d ; P)}{d} \sum_{n \leqslant \min \left\{B / d, B \sqrt{\|P\|} / d^{3 / 2}\right\}} \frac{f_{d}(n)}{n} \\
& =\frac{1}{8} B \operatorname{vol}\left(\mathcal{R}_{P}\right) \sum_{d \leqslant D} \frac{\mu(d) \chi(d) r(d) \varphi^{\dagger}(d) W^{*}(d ; P)}{d}\{\log B+O(\log (2 d))\} \\
& =\left\{C_{P}+O\left(1 / D^{1-\varepsilon}\right)\right\} B \operatorname{vol}\left(\mathcal{R}_{P}\right) \log B,
\end{aligned}
$$

pour chaque $\varepsilon>0$ fixé, avec

$$
C_{P}:=\frac{1}{8} \sum_{d \in \mathbb{N}^{*}} \frac{\mu(d) \chi(d)^{2} r(d) \varphi^{\dagger}(d) W^{*}(d ; P)}{d} .
$$

En choisissant $\varepsilon$ assez petit, nous en déduisons l'estimation

$$
N_{P}(B)=C_{P} \operatorname{vol}\left(\mathcal{R}_{P}\right) B \log B+O\left(B(\log B)^{96 / 97}\right),
$$

d'où $(8 \cdot 1)$, avec un peu de marge.

\subsection{Validation de la conjecture de Peyre}

Nous nous proposons ici de montrer l'égalité

$$
C_{P} \operatorname{vol}\left(\mathcal{R}_{P}\right)=C_{H}(V) \text {. }
$$

D'après les calculs effectués dans [16], la valeur $C_{H}(V)$ conjecturée par Peyre pour le terme principal de la formule asymptotique $(8 \cdot 1)$ constante s'écrit

$$
C_{H}(V)=\omega_{\infty} \prod_{p} \omega_{p}
$$

où $\omega_{\infty}$ et $\omega_{p}$ désignent respectivement les volumes relatifs aux densités archimédienne et $p$-adique des sous-domaines de la boule de rayon $B$ dans $\mathbb{R}^{5}$ limités par la surface $V$.

Commençons par le calcul des différents facteurs apparaissant dans (8.18). Pour tout nombre premier $p$, nous avons

$$
\omega_{p}:=\lim _{\nu \rightarrow \infty} \frac{1}{p^{4 \nu}} \sum_{\substack{(u, v, x, y, t) \in\left(\mathbb{Z} / p^{\nu} \mathbb{Z}\right)^{5} \\ P(u, v) t^{2} \equiv x^{2}+y^{2}\left(\bmod p^{\nu}\right) \\ p \nmid(u, v), p \nmid(x, y, t)}} 1 .
$$

Pour évaluer cette limite, nous faisons appel au calcul des quantités

$$
N\left(p^{\nu}, a\right):=\left|\left\{(x, y) \in\left(\mathbb{Z} / p^{\nu} \mathbb{Z}\right)^{2}: x^{2}+y^{2} \equiv a\left(\bmod p^{\nu}\right)\right\}\right| \quad(p \in \mathcal{P}, a \in \mathbb{N}),
$$

où $\mathcal{P}$ désigne l'ensemble des nombres premiers. 
Rappelons la définition de l'ensemble $\mathcal{E}$ en $(7 \cdot 1)$. Nous avons (voir par exemple [2])

$$
N\left(p^{\nu}, a\right)= \begin{cases}p^{\nu}(1+1 / p) & \text { si } p \equiv 3(\bmod 4), \nu_{p}(a)<\nu \text { et } 2 \mid a \\ \left\{1+v_{p}(a)\right\} p^{\nu}(1-1 / p) & \text { si } p \equiv 1(\bmod 4), \nu_{p}(a)<\nu \\ 2^{\nu+1} & \text { si } a \in \mathcal{E}\end{cases}
$$

Comme $(t, p)=1$ dans $(8 \cdot 19)$, il s'ensuit que, lorsque $p \equiv 3(\bmod 4)$, nous avons

$$
\begin{aligned}
\omega_{p} & =\lim _{\nu \rightarrow \infty} \frac{1-1 / p}{p^{3 \nu}} \sum_{\substack{(u, v, x, y, t) \in\left(\mathbb{Z} / p^{\nu} \mathbb{Z}\right)^{5} \\
P(u, v) \equiv x^{2}+y^{2}\left(\bmod p^{\nu}\right) \\
p \nmid(u, v)}} 1 \\
& =\left(1-\frac{1}{p^{2}}\right)\left\{1-\frac{1}{p^{2}}-\frac{\varrho_{P}^{*}(p)}{p^{2}}+\sum_{\substack{k \geqslant 2 \\
2 \mid k}} \frac{\varrho_{P}^{*}\left(p^{k}\right)}{p^{2 k}}-\frac{\varrho_{P}^{*}\left(p^{k+1}\right)}{p^{2 k+2}}\right\} \\
& =\left(1-\frac{1}{p^{2}}\right)\left\{1-\frac{1}{p^{2}}+\sum_{\nu \geqslant 1} \frac{\chi\left(p^{\nu}\right) \varrho_{P}^{*}\left(p^{\nu}\right)}{p^{2 \nu}}\right\} .
\end{aligned}
$$

Pour plus de détails sur ce type de calcul, le lecteur pourra consulter le paragraphe 2 de $[4]$.

Lorsque $p \equiv 1(\bmod 4)$, désignons, pour $\ell \geqslant 0$, par $\omega_{p}(\ell)$ la contribution à $\omega_{p}$ des quintuplets $(u, v, x, y, t)$ tels que $v_{p}(t)=\ell$. Il résulte alors de $(8 \cdot 20)$ que

$$
\begin{aligned}
\omega_{p}(0) & =\left(1-\frac{1}{p}\right)^{2}\left\{1-\frac{1}{p^{2}}-\frac{\varrho_{P}^{*}(p)}{p^{2}}+\sum_{k \geqslant 1}(k+1)\left(\frac{\varrho_{P}^{*}\left(p^{k}\right)}{p^{2 k}}-\frac{\varrho_{P}^{*}\left(p^{k+1}\right)}{p^{2 k+2}}\right)\right\} \\
& =\left(1-\frac{1}{p}\right)^{2}\left\{1-\frac{1}{p^{2}}+\sum_{\nu \geqslant 1} \frac{\varrho_{P}^{*}\left(p^{\nu}\right)}{p^{2 \nu}}\right\},
\end{aligned}
$$

et

$$
\begin{aligned}
\omega_{p}(\ell) & =\frac{(1-1 / p)^{2}}{p^{\ell}}\left\{1-\frac{1}{p^{2}}-\frac{\varrho_{P}^{*}(p)}{p^{2}}+\sum_{k \geqslant 1} 2\left(\frac{\varrho_{P}^{*}\left(p^{k}\right)}{p^{2 k}}-\frac{\varrho_{P}^{*}\left(p^{k+1}\right)}{p^{2 k+2}}\right)\right\} \\
& =2 \frac{(1-1 / p)^{2}}{p^{\ell}}\left(1-\frac{1}{p^{2}}\right) \quad(\ell \geqslant 1),
\end{aligned}
$$

d'où

$$
\omega_{p}=\left(1-\frac{1}{p^{2}}\right)^{2}+\left(1-\frac{1}{p}\right)^{2} \sum_{\nu \geqslant 1} \frac{\chi\left(p^{\nu}\right) \varrho_{P}^{*}\left(p^{\nu}\right)}{p^{2 \nu}} \quad(p \equiv 1(\bmod 4)) .
$$

Pour calculer $\omega_{2}$, nous observons que les conditions de sommations dans (8.19) impliquent $v_{2}(t)=0$. Il y a $2^{\nu-1}$ nombres impairs dans $\left[1,2^{\nu}\right]$. Il résulte donc de $(8 \cdot 20)$ que

$$
\omega_{2}=\lim _{\nu \rightarrow \infty} \frac{1}{2^{3 \nu+1}} \sum_{\substack{(u, v, x, y) \in\left(\mathbb{Z} / 2^{\nu} \mathbb{Z}\right)^{4} \\ P(u, v) \equiv x^{2}+y^{2}\left(\bmod 2^{\nu}\right) \\ 2 \nmid(u, v)}} 1=\lim _{\nu \rightarrow \infty} \frac{1}{2^{2 \nu}} \sum_{\substack{(u, v) \in\left(\mathbb{Z} / 2^{\nu} \mathbb{Z}\right)^{2} \\ P\left(u, v \in \mathcal{E}\left(\bmod 2^{\nu}\right) \\ 2 \nmid(u, v)\right.}} 1 .
$$

Montrons ensuite la formule

$$
\omega_{\infty}=\frac{1}{2} \pi \operatorname{vol}\left(\mathcal{R}_{P}\right) .
$$


Grâce à la symétrie du problème, nous pouvons nous restreindre à évaluer une intégrale sur le premier quadrant $x>0$ et $y>0$. Il suit

$$
\omega_{\infty}=2 \lim _{B \rightarrow \infty} \frac{1}{B \log B} \int_{\mathcal{D}} \frac{\mathrm{d} u \mathrm{~d} v \mathrm{~d} t \mathrm{~d} y}{2 \sqrt{t^{2} P(u, v)-y^{2}}}
$$

avec

$$
\mathcal{D}:=\left\{(u, v, t, y) \in \mathbb{R}^{4}:(u, v) \in \mathcal{R}_{P}(\sqrt{B / t}), 0<y<t \sqrt{P(u, v)}, 1<t<B\right\}
$$

En faisant appel à la formule (7.9), nous obtenons

$$
\begin{aligned}
\omega_{\infty} & =\frac{1}{2} \pi \lim _{B \rightarrow \infty} \frac{1}{B \log B} \int_{1}^{B} \mathrm{~d} t \int_{\mathcal{R}_{P}(\sqrt{B / t})} \mathrm{d} u \mathrm{~d} v \\
& =\frac{1}{2} \pi \operatorname{vol}\left(\mathcal{R}_{P}\right) \lim _{B \rightarrow \infty} \frac{1}{\log B} \int_{1}^{B} \frac{\mathrm{d} t}{t}=\frac{1}{2} \pi \operatorname{vol}\left(\mathcal{R}_{P}\right) .
\end{aligned}
$$

Nous sommes à présent en mesure d'effectuer la vérification annoncée. La constante $C_{P}$ apparaissant dans $(8 \cdot 1)$ est définie en $(8 \cdot 16)$. Compte tenu de $(2 \cdot 12),(8 \cdot 10)$ et $(8 \cdot 14)$, nous obtenons la représentation

$$
C_{P}=\frac{\pi}{16} \prod_{p}\left(1+\frac{\chi(p)}{p}\right) \sum_{d \geqslant 1} \frac{\mu(d) \chi(d)^{2} r(d) \varphi^{\dagger}(d) W^{*}(d ; P)}{d}=\frac{\pi}{2} \prod_{p} c_{p}
$$

où l'on a posé

$$
c_{2}:=\frac{1}{2} W_{2}^{*}(1 ; P), \quad c_{p}:=\left(1+\frac{\chi(p)}{p}\right)\left\{W_{p}^{*}(1 ; P)-\frac{r(p) W_{p}^{*}(p ; P)}{4(p+1)}\right\} \quad(p>2) .
$$

Les formules $(8 \cdot 11),(8 \cdot 12)$ et $(8 \cdot 14)$ nous permettent donc de calculer les $c_{p}$. Nous allons en fait établir que l'on a

$$
c_{p}=\omega_{p} \quad(p \in \mathcal{P}),
$$

ce qui, compte tenu de (8.24), suffira à prouver $(8 \cdot 17)$.

Pour $p=2$, cela découle immédiatement de (8.23) au vu de (8.12).

Lorsque $p \equiv 3 \bmod 4$, nous avons $r(p)=0$ et donc, d'après $(8 \cdot 14)$,

$$
c_{p}=\left(1-\frac{1}{p^{2}}\right)\left\{1-\frac{1}{p^{2}}+\sum_{\nu \geqslant 1} \frac{\chi\left(p^{\nu}\right) \varrho_{P}^{*}\left(p^{\nu}\right)}{p^{2 \nu}}\right\} .
$$

La formule escomptée résulte donc de $(8 \cdot 21)$.

Lorsque $p \equiv 1 \bmod 4$, nous avons $\chi(p)=1$ et $r(p)=8$, donc

$$
\begin{aligned}
c_{p} & =\left(1-\frac{1}{p^{2}}\right)\left\{1-\frac{1}{p^{2}}+\sum_{\nu \geqslant 1} \frac{\varrho_{P}^{*}\left(p^{\nu}\right)}{p^{2 \nu}}-\frac{2}{p+1} \sum_{\nu \geqslant 0} \frac{\varrho_{P}^{*}\left(p^{\nu+1}\right)}{p^{2 \nu+2}}\right\} \\
& =\left(1-\frac{1}{p^{2}}\right)^{2}+\left(1-\frac{1}{p}\right)^{2} \sum_{\nu \geqslant 1} \frac{\varrho_{P}^{*}\left(p^{\nu}\right)}{p^{2 \nu}}=\omega_{p},
\end{aligned}
$$

d'après $(8 \cdot 22)$.

Cela achève la preuve de $(8 \cdot 17)$. 


\section{Le cas $P=P_{1} P_{2}:$ preuve du Théorème 1.2}

\subsection{Présentation}

Nous précisons ici le schéma de la démonstration du Théorème 1.2.

Supposant que la forme binaire $P$ se décompose sous la forme $P=P_{1} P_{2}$ où $P_{1}$ et $P_{2}$ sont de degré 2 et irréductibles sur $\mathbb{Q}[i]$, nous notons

$$
\Delta:=\left|\operatorname{Res}\left(P_{1}, P_{2}\right)\right|
$$

le résultant de $P_{1}$ et $P_{2}$, de sorte que $q \mid \operatorname{pgcd}\left(P_{1}(\boldsymbol{x}), P_{2}(\boldsymbol{x})\right)$ avec $\boldsymbol{x}=(m, n)$ et $\operatorname{pgcd}(m, n)=1$ implique $q \mid \Delta$.

L'étude asymptotique de l'expression $(2 \cdot 6)$ pour $N_{P}(B)$ nécessite une estimation des sommes $S_{P}$ définies en $(2 \cdot 5)$. À cette fin, pour chaque vecteur $\boldsymbol{x} \in \mathbb{Z}^{2}$ tel que $(m, n)=1$ et chaque diviseur fixé $d$ sans facteur carré de $P(\boldsymbol{x})=P_{1}(\boldsymbol{x}) P_{2}(\boldsymbol{x})$, nous insérons la formule

$$
r\left(\frac{P_{1}(\boldsymbol{x}) P_{2}(\boldsymbol{x})}{d}\right)=\frac{1}{2^{\omega\left(\left(d, P_{1}(\boldsymbol{x}), P_{2}(\boldsymbol{x})\right)\right)}} \sum_{\substack{\left(t_{1}, t_{2}\right) \in \mathbb{N}^{* 2} \\ t_{1} t_{2}=d \\ t_{1}\left|P_{1}(\boldsymbol{x}), t_{2}\right| P_{2}(\boldsymbol{x})}} r\left(\frac{P_{1}(\boldsymbol{x}) P_{2}(\boldsymbol{x})}{t_{1} t_{2}}\right) .
$$

Lorsque $\varepsilon \in X:=\{-1,1\}$, nous posons, en cohérence avec $(2 \cdot 1)$,

$$
\mathcal{R}_{P, \varepsilon}:=\left\{\boldsymbol{x} \in \mathbb{R}^{2}:\|x\|_{\infty}<1, \varepsilon P_{1}(\boldsymbol{x})>0, \varepsilon P_{2}(\boldsymbol{x})>0\right\} .
$$

Rappelant la notation $(3 \cdot 3)$ pour les ensembles $\Lambda(\boldsymbol{s} ; \boldsymbol{T})$, nous définissons en outre, pour tous $\boldsymbol{d}, \boldsymbol{D} \in \mathbb{N}^{* 2}$ tels que $d_{j} \mid D_{j}(j=1,2)$, tout vecteur $\boldsymbol{T}:=\left(T_{1}, T_{2}\right)$, et tout domaine $\mathcal{R}$ de $\mathbb{R}^{2}$ satisfaisant $\left(H_{1}\right)$ et tel que $\left.T_{i}(\mathcal{R}) \subset\right] 0, \infty[(i=1,2)$,

$$
\mathcal{Q}_{\boldsymbol{T}}^{*}(\xi, \boldsymbol{d}, \boldsymbol{D} ; \mathcal{R}):=\sum_{\substack{\boldsymbol{x} \in \Lambda(\boldsymbol{D} ; \boldsymbol{T}) \cap \mathcal{R}(\xi) \\(m, n)=1}} r\left(\frac{T_{1}(\boldsymbol{x})}{d_{1}}\right) r\left(\frac{T_{2}(\boldsymbol{x})}{d_{2}}\right) .
$$

En insérant alors $(2 \cdot 10)$ dans $(2 \cdot 6)$, nous obtenons

$(9 \cdot 3)$

$$
N_{P}(B)=\frac{1}{2^{5}} \sum_{d \leqslant B} \mu(d) \chi(d) \sum_{n \leqslant \min \left\{B / d, B \sqrt{\left.\|P\| / d^{3 / 2}\right\}}\right.} f_{d}(n) \sum_{\substack{\boldsymbol{t} \in \mathbb{N}^{* 3} \\ t_{1} t_{2}=d, t_{3} \mid \Delta}} \chi\left(t_{3}\right) \mu\left(t_{3}\right) S_{P_{1}, P_{2}}\left(\sqrt{\frac{B}{d n}}, \boldsymbol{t}\right),
$$

où l'on a posé

$$
\begin{array}{r}
S_{P_{1}, P_{2}}(\xi, \boldsymbol{t}):=\sum_{\varepsilon \in \mathcal{X}} \sum_{\substack{\boldsymbol{x} \in \Lambda\left(t_{1} t_{3}, t_{2} t_{3} ; P_{1}, P_{2}\right) \cap \mathcal{R}_{P, \boldsymbol{\varepsilon}}(\xi) \\
(m, n)=1}} \frac{r\left(\varepsilon P_{1}(\boldsymbol{x}) / t_{1} t_{3}\right) r\left(\varepsilon P_{2}(\boldsymbol{x}) / t_{2} t_{3}\right)}{2^{\omega\left(\left(d, P_{1}(\boldsymbol{x}), P_{2}(\boldsymbol{x})\right)\right)}} \\
=\sum_{\varepsilon \in \mathcal{X}} \sum_{k k^{\prime} \mid(\Delta, d)} \frac{\mu\left(k^{\prime}\right)}{2^{\omega(k)}} \mathcal{Q}_{\varepsilon P_{1}, \varepsilon P_{2}}^{*}\left(\xi,\left(t_{1} t_{3}, t_{2} t_{3}\right),\left(\left[t_{1} t_{3}, k k^{\prime}\right],\left[t_{2} t_{3}, k k^{\prime}\right]\right) ; \mathcal{R}_{P, \varepsilon}\right) .
\end{array}
$$

Une inversion de Möbius permet de disposer de la condition $(m, n)=1$ dans la somme $\mathcal{Q}_{\boldsymbol{T}}^{*}\left(\xi, \boldsymbol{d}, \boldsymbol{D} ; \mathcal{R}_{P, \varepsilon}\right)$. Nous pouvons donc nous contenter d'estimer

$$
\mathcal{Q}_{\boldsymbol{T}}(\xi, \boldsymbol{d}, \boldsymbol{D} ; \mathcal{R}):=\sum_{\boldsymbol{x} \in \Lambda(\boldsymbol{D} ; \boldsymbol{T}) \cap \mathcal{R}(\xi)} r\left(\frac{T_{1}(\boldsymbol{x})}{d_{1}}\right) r\left(\frac{T_{2}(\boldsymbol{x})}{d_{2}}\right)
$$


où $\mathcal{R}$ est un domaine de $\mathbb{R}^{2}$ vérifiant $\left(H_{1}\right)$ et $T_{1}, T_{2}$ sont des formes binaires quadratiques irréductibles sur $\mathbb{Q}[i]$ telles que $\left.T_{j}(\mathcal{R}) \subset\right] 0, \infty[(j=1,2)$.

\subsection{Sommes de carrés localisées : énoncé des formules asymptotiques}

Nous verrons au $\S 9.4$ comment une manipulation de routine sur les réseaux couplée à un changement de variables permet de réduire l'estimation de la quantité $(9 \cdot 5)$ à celle de

$$
\mathcal{Q}(\xi ; \boldsymbol{T}, \mathcal{R})=\mathcal{Q}_{\boldsymbol{T}}(\xi,(1,1),(1,1) ; \mathcal{R})=\sum_{\boldsymbol{x} \in \mathbb{Z}^{2} \cap \mathcal{R}(\xi)} r\left(T_{1}(\boldsymbol{x})\right) r\left(T_{2}(\boldsymbol{x})\right) .
$$

Il s'agit donc de démontrer le pendant du Lemme 7.1. Par analogie avec les hypothèses $\left(H_{2}\right)$ et $\left(H_{3}\right)$ considérées au $\S 5.2$, nous introduisons les conditions :

$\left(H_{2}^{\prime}\right) \quad T_{1}$ et $T_{2}$ sont des formes binaires de $\mathbb{Z}[X, Y]$ irréductibles sur $\mathbb{Q}[i]$ de degré 2 non proportionnelles;

$\left.\left(H_{3}^{\prime}\right) \quad T_{1}(\mathcal{R}) \subset\right] 0, \infty\left[, T_{2}(\mathcal{R}) \subset\right] 0, \infty[$.

Nous posons alors, parallèlement à $(5 \cdot 3)$,

$$
\delta_{\boldsymbol{T}}=\delta_{\boldsymbol{T}}(\mathcal{R}):=\sup _{\boldsymbol{x} \in \mathcal{R}} \max _{j=1,2}\left|T_{j}(\boldsymbol{x})\right|^{1 / 2},
$$

et retenons la notation $\delta_{\infty}(\mathcal{R})$ de $(5 \cdot 3)$.

Enfin, pour $\boldsymbol{T}=\left(T_{1}, T_{2}\right)$, nous posons $\|\boldsymbol{T}\|:=\max \left\{\left\|T_{1}\right\|,\left\|T_{2}\right\|\right\}$ et rappelons la notation $\mathcal{E}_{\nu}$ introduite en $(7 \cdot 3)$.

Lemme 9.1. Soient $\varepsilon>0, \kappa>0, J_{1}, J_{2} \in \mathbb{Z}[X, Y]$ des formes binaires de degré 2 irréductibles sur $\mathbb{Q}[i], M \in M_{2}(\mathbb{Z})^{*}, q_{1}, q_{2} \in \mathbb{N}^{*}$, tels que $2 \nmid q_{1} q_{2}$ dét $M$, et $\mathcal{R}$ un domaine de $\mathbb{R}^{2}$ vérifiant $\left(H_{1}\right)$. Sous l'hypothèse que les formes binaires $T_{i}(i=1,2)$ définies par $T_{i}(\boldsymbol{x}):=J_{i}(M \boldsymbol{x}) / q_{i}\left(\boldsymbol{x} \in \mathbb{R}^{2}\right)$ sont à coefficients entiers et vérifient $\left(H_{2}^{\prime}\right)$ et $\left(H_{3}^{\prime}\right)$, et sous les conditions

$$
\begin{aligned}
& \xi \geqslant \mathrm{e}^{\kappa\left(q_{1}+q_{2}\right)}+\|M\|^{\kappa}, \quad 1 / \sqrt{\xi} \leqslant \sigma \leqslant \xi^{3 / 2}, \quad 1 / \sqrt{\xi} \leqslant \vartheta \leqslant \xi^{3 / 2}, \\
& \delta_{\infty}(\mathcal{R}) \leqslant \sigma, \quad \delta_{\boldsymbol{T}}(\mathcal{R}) \leqslant \vartheta, \quad \vartheta \leqslant\|\boldsymbol{T}\|^{1 / 4} \sigma
\end{aligned}
$$

nous avons

$$
\mathcal{Q}(\xi ; \boldsymbol{T}, \mathcal{R})=\pi^{2} \mathcal{K}(\boldsymbol{T}) \operatorname{vol}(\mathcal{R}) \xi^{2}+O\left(\frac{\|\boldsymbol{T}\|^{\varepsilon} \xi^{2}(\sigma+\vartheta)^{2}}{(\log \xi)^{3 / 104}}\right)
$$

où $\mathcal{K}(\boldsymbol{T}):=\prod_{p} \mathcal{K}_{p}(\boldsymbol{T})$ et

$$
\begin{aligned}
\mathcal{K}_{2}(\boldsymbol{T}) & :=\lim _{\nu \rightarrow \infty} 2^{-2 \nu+2}\left|\left\{\boldsymbol{x} \in\left(\mathbb{Z} / 2^{\nu} \mathbb{Z}\right)^{2}: T_{i}(\boldsymbol{x}) \in \mathcal{E}_{\nu}(i=1,2)\right\}\right| \\
\mathcal{K}_{p}(\boldsymbol{T}) & :=\left(1-\frac{\chi(p)}{p}\right)^{2} \sum_{\boldsymbol{\nu} \in \mathbb{N}^{2}} \chi\left(p^{\nu_{1}+\nu_{2}}\right) \frac{\varrho_{\boldsymbol{T}}\left(p^{\nu_{1}}, p^{\nu_{2}}\right)}{p^{2 \nu_{1}+2 \nu_{2}}} \quad(p>2) .
\end{aligned}
$$

De plus, pour tout nombre premier $p$, nous avons

$(9 \cdot 10) \mathcal{K}_{p}(\boldsymbol{T})=\lim _{\nu \rightarrow \infty} p^{-4 \nu}\left|\left\{(\boldsymbol{x}, \boldsymbol{s}, \boldsymbol{t}) \in\left(\mathbb{Z} / p^{\nu} \mathbb{Z}\right)^{6}: T_{i}(\boldsymbol{x}) \equiv s_{i}^{2}+t_{i}^{2}\left(\bmod p^{\nu}\right) \quad(i=1,2)\right\}\right|$.

La somme $Q(\xi ; \boldsymbol{T}, \mathcal{R})$ dénombre les solutions entières du système diophantien

$$
s_{i}^{2}+t_{i}^{2}=T_{i}(m, n) \quad(i=1,2)
$$


sous la condition $\boldsymbol{x}=(m, n) \in \mathcal{R}(\xi)$. Comme dans le cas de la constante $K(T)$ apparaissant dans $(7 \cdot 5)$, il est possible de montrer que $\mathcal{K}(\boldsymbol{T})$ est le produit des densités $p$-adiques associées et que la densité archimédienne associée est bien égale à $\pi^{2} \operatorname{vol}(\mathcal{R})$.

Il est à noter que le système $(9 \cdot 11)$ correspond à l'intersection de deux quadriques dans l'espace affine de dimension 6. La possibilité d'une évaluation asymptotique du nombre des solutions entières représente donc un progrès significatif dans les problèmes de cette catégorie. En effet, à ce jour, la mise en œuvre de la méthode du cercle, qui est l'outil standard pour ce type de résultat, nécessite trois hypothèses supplémentaires : que les formes quadratiques soient diagonales, que l'intersection des deux quadriques soit non singulière, et que le nombre total des variables soit au moins égal à 9 — cf. [14].

\section{3. Preuve du Lemme 9.1}

Comme la structure de la démonstration est semblable à celle du Lemme 7.1, nous omettrons certains détails.

Nous commençons par estimer la constante $\mathcal{K}(\boldsymbol{T})$.

Lemme 9.2. Dans les hypothèses du Lemme 9.1,

$$
\mathcal{K}(\boldsymbol{T}) \ll\left\{\log _{2}\left(2+q_{1} q_{2}\|\boldsymbol{T}\|\right)\right\}^{50},
$$

où la constante implicite dépend au plus de $\varepsilon, \kappa, J_{1}, J_{2}$.

Démonstration. Pour chaque nombre premier impair $p$, désignons par $\mathcal{E}_{p}(\boldsymbol{T})$ et $\mathcal{F}_{p}(\boldsymbol{T})$ les contributions respectives à la somme

$$
\sum_{\left(\nu_{1}, \nu_{2}\right) \in \mathbb{N}^{2}} \chi\left(p^{\nu_{1}+\nu_{2}}\right) \frac{\varrho_{T}\left(p^{\nu_{1}}, p^{\nu_{2}}\right)}{p^{2 \nu_{1}+2 \nu_{2}}}
$$

des couples $\left(\nu_{1}, \nu_{2}\right)$ tels que $1 \leqslant \nu_{1}, \nu_{2} \leqslant 2$ et $\max \left(\nu_{1}, \nu_{2}\right) \geqslant 3$, de sorte que

$$
\mathcal{K}_{p}(\boldsymbol{T})=\left(1-\frac{\chi(p)}{p}\right)^{2}\left\{1+\mathcal{E}_{p}(\boldsymbol{T})+\mathcal{F}_{p}(\boldsymbol{T})\right\} \quad(p>2)
$$

Majorons $\mathcal{F}_{p}(\boldsymbol{T})$. D'après $(3 \cdot 5)$, nous pouvons écrire pour tous entiers $\nu_{1} \geqslant 0, \nu_{2} \geqslant 0$

$$
\varrho_{\boldsymbol{T}}\left(p^{\nu_{1}}, p^{\nu_{2}}\right) \leqslant \min \left\{p^{2 \nu_{1}} \varrho_{T_{2}}^{+}\left(p^{\nu_{2}}\right), p^{2 \nu_{2}} \varrho_{T_{1}}^{+}\left(p^{\nu_{1}}\right)\right\} \leqslant 5 p^{2 \nu_{1}+2 \nu_{2}-\max \left\{\left\lceil\nu_{1} / 2\right\rceil,\left\lceil\nu_{2} / 2\right\rceil\right\}},
$$

donc

$$
\mathcal{F}_{p}(\boldsymbol{T}) \ll 1 / p^{2},
$$

où la constante implicite est absolue.

Rappelons la définition des ensembles $\Lambda(\boldsymbol{s} ; \boldsymbol{T})$ en $(3 \cdot 3)$ et posons

$$
\Lambda^{*}(\boldsymbol{s} ; \boldsymbol{T}):=\left\{(m, n) \in \Lambda(s ; \boldsymbol{T}):\left(m, n, s_{1} s_{2}\right)=1\right\}, \quad \varrho_{\boldsymbol{T}}^{*}(\boldsymbol{s}):=\mid \Lambda^{*}(\boldsymbol{s} ; \boldsymbol{T}) \cap\left[0,\left.s_{1} s_{2}\right|^{2} \mid .\right.
$$

En classant les couples $(m, n)$ comptés dans $\varrho_{\boldsymbol{T}}\left(p^{\nu_{1}}, p^{\nu_{2}}\right)$ selon la valeur de

$$
v_{p}\left(\left(m, n, p^{\max \left\{\left\lceil\nu_{1} / 2\right\rceil,\left\lceil\nu_{2} / 2\right\rceil\right\}}\right)\right),
$$

nous obtenons la relation générale

$$
\varrho_{\boldsymbol{T}}\left(p^{\nu_{1}}, p^{\nu_{2}}\right)=\sum_{0 \leqslant k \leqslant \max \left\{\left\lceil\nu_{1} / 2\right\rceil,\left\lceil\nu_{2} / 2\right\rceil\right\}} \varrho_{\boldsymbol{T}}^{*}\left(p^{\left(\nu_{1}-2 k\right)^{+}}, p^{\left(\nu_{2}-2 k\right)^{+}}\right) p^{m_{k}} \quad\left(\nu_{1} \geqslant 1, \nu_{2} \geqslant 1\right)
$$

où l'on a posé $m_{k}:=2 \min \left\{\nu_{1}, 2 k\right\}+2 \min \left\{\nu_{2}, 2 k\right\}-2 k$. En particulier, nous avons

$$
\varrho_{\boldsymbol{T}}\left(p^{\nu_{1}}, p^{\nu_{2}}\right)=\varrho_{\boldsymbol{T}}^{*}\left(p^{\nu_{1}}, p^{\nu_{2}}\right)+p^{2 \nu_{1}+2 \nu_{2}-2} \quad\left(1 \leqslant \nu_{1}, \nu_{2} \leqslant 2\right),
$$

le premier terme du membre de droite étant nul si $p$ ne divise pas le résultant $\operatorname{Res}\left(T_{1}, T_{2}\right)$. 
Lorsque $p \nmid \operatorname{disc}\left(T_{j}\right)(j=1,2)$, nous avons, d'après le Lemme $3.2, \varrho_{T_{j}}^{+}\left(p^{\nu}\right) \leqslant 5 p^{\nu}$. Enfin, rappelons que $\operatorname{Res}\left(T_{1}, T_{2}\right) \operatorname{disc}\left(T_{1}\right) \operatorname{disc}\left(T_{2}\right) \mid D^{*}$ où $D^{*}:=\operatorname{disc}\left(T_{1} T_{2}\right)$. Lorsque $p \nmid D^{*}$, nous obtenons donc

$$
\begin{aligned}
\mathcal{K}_{p}(\boldsymbol{T}) & =\left(1-\frac{\chi(p)}{p}\right)^{2}\left(1+\sum_{\substack{\nu \in\{1,2\} \\
j=1,2}} \chi\left(p^{\nu}\right) \frac{\varrho_{T_{j}}^{+}\left(p^{\nu}\right)}{p^{2 \nu}}+O\left(\frac{1}{p^{2}}\right)\right) \\
& =1+\sum_{j=1,2} \chi(p) \frac{\varrho_{T_{j}}^{+}(p)-p}{p^{2}}+O\left(\frac{1}{p^{2}}\right)
\end{aligned}
$$

alors qu'une majoration grossière à partir du Lemme 3.2 fournit, pour tout nombre premier $p$,

$$
\mathcal{K}_{p}(\boldsymbol{T}) \leqslant 1+\frac{42}{p}+O\left(\frac{1}{p^{2}}\right)
$$

où la constante implicite est absolue. Nous concluons en remarquant que $\varrho_{T_{j}}^{+}(p)=\varrho_{J_{j}}^{+}(p)$ lorsque $p \nmid$ dét $M$. En effet, notant que $\mathcal{K}_{2}(\boldsymbol{T}) \leqslant 4$, nous obtenons

$$
\begin{aligned}
& \prod_{p} \mathcal{K}_{p}(\boldsymbol{T}) \ll \prod_{p \nmid 2 D^{*} \operatorname{dét~} M} \mathcal{K}_{p}(\boldsymbol{T}) \prod_{p \mid D^{*} \operatorname{dét} M}\left(1+\frac{42}{p}\right) \\
& \leqslant \prod_{p}\left\{1+\sum_{j=1,2} \chi(p) \frac{\varrho_{J_{j}}^{+}(p)-p}{p^{2}}+O\left(\frac{1}{p^{2}}\right)\right\} \prod_{p \mid D^{*} \operatorname{dét~} M}\left(1+\frac{50}{p}\right) \\
& \ll J_{1}, J_{2} \\
& \prod_{p \mid D^{*} \text { dét } M}\left(1+\frac{50}{p}\right) \ll\left\{\log _{2}\left(2+q_{1} q_{2}\|\boldsymbol{T}\|\right)\right\}^{50},
\end{aligned}
$$

où nous avons utilisé le Lemme 3.3 pour majorer le premier produit de la deuxième ligne et, pour la dernière estimation, pris en compte l'identité

$$
(\text { dét } M)^{2} \text { dét } J_{i}=q_{i}^{4} \text { dét } T_{i} \quad(i=1,2) .
$$

Nous pouvons dorénavant supposer

$$
\|\boldsymbol{T}\| \leqslant(\log \xi)^{1 / \varepsilon} .
$$

En effet, lorsque cette condition n'est pas réalisée, il résulte du Lemme 9.2 que le terme principal de (9.9) peut être absorbé par le terme résiduel. La formule (9.9) découle alors trivialement de la majoration

$$
\mathcal{Q}(\xi ; \boldsymbol{T}, \mathcal{R}) \ll\|\boldsymbol{T}\|^{\varepsilon / 2} \sigma^{2} \xi^{2}
$$

issue du Théorème 4.2 et du Lemme 4.3.

La réduction $(9 \cdot 12)$ étant opérée, un second indispensable préalable à la preuve du Lemme 9.1 consiste à établir un résultat de niveau de répartition analogue au Lemme 5.2. Il s'agit donc de majorer, pour $\boldsymbol{y}=\left(y_{1}, y_{2}\right) \in\left[2, \infty\left[^{2}\right.\right.$ et $\xi$ assez grand,

$$
\Phi(\xi, \boldsymbol{y} ; \boldsymbol{T}, \sigma, \vartheta):=\sum_{\substack{1 \leqslant t_{j} \leqslant y_{j}(j=1,2) \\ 2 \nmid t_{1} t_{2}}} \sup _{\mathcal{R}}|| \Lambda(\boldsymbol{t} ; \boldsymbol{T}) \cap \mathcal{R}^{\dagger}(\xi)\left|-\operatorname{vol}(\mathcal{R}) \xi^{2} \frac{\varrho_{\boldsymbol{T}}(\boldsymbol{t})}{2 t_{1}^{2} t_{2}^{2}}\right|,
$$

où le supremum est pris sur l'ensemble des parties convexes $\mathcal{R}$ de $\mathbb{R}^{2}$ telles que $\delta_{\infty}(\mathcal{R}) \leqslant \sigma$, $\delta_{T}(\mathcal{R}) \leqslant \vartheta$, et dont la longueur de la frontière est $\ll \sigma$.

Nous nous contentons d'énoncer le résultat que nous utiliserons. Il est à noter à ce stade que, contrairement à la situation considérée au Lemme 7.1, un facteur supplémentaire $\ll(\log \xi)^{O(1)}$ est ici acceptable dans le majorant. Le lecteur pourra se reporter à [25] où est établie une version faible, non uniforme dans les coefficients de $\boldsymbol{T}$, d'un résultat de même nature. 
Lemme 9.3. Soient $\varepsilon>0, \kappa>0, J_{1}, J_{2} \in \mathbb{Z}[X, Y]$ deux formes binaires irréductibles sur $\mathbb{Q}$ de degré $2, M \in M_{2}(\mathbb{Z})^{*}, q_{1}, q_{2} \in \mathbb{N}^{*}$, et $\mathcal{R}$ un domaine de $\mathbb{R}^{2}$ vérifiant $\left(H_{1}\right)$. Sous l'hypothèse que les formes binaires $T_{1}, T_{2}$ définies par $T_{i}(\boldsymbol{x})=J_{i}(M \boldsymbol{x}) / q_{i}$ $\left(\boldsymbol{x} \in \mathbb{R}^{2}, i=1,2\right)$ sont à coefficients entiers et vérifient $\left(H_{2}^{\prime}\right)$ et $\left(H_{3}^{\prime}\right)$ et sous les conditions

$$
\begin{aligned}
& y_{1}, y_{2} \geqslant 2, \quad \xi \geqslant \mathrm{e}^{\kappa\left(q_{1}+q_{2}\right)}+\|M\|^{\kappa}, \\
& 1 / \sqrt{\xi} \leqslant \sigma \leqslant \xi^{3 / 2}, \quad 1 / \sqrt{\xi} \leqslant \vartheta \leqslant \xi^{3 / 2}, \quad \vartheta \leqslant\|\boldsymbol{T}\|^{1 / 4} \sigma,
\end{aligned}
$$

nous avons

$$
\Phi(\xi, \boldsymbol{y} ; \boldsymbol{T}, \sigma, \vartheta) \ll\|\boldsymbol{T}\|^{\varepsilon}(\log \xi)^{2}\left\{\sigma \xi \sqrt{y_{1} y_{2}}+y_{1} y_{2}\right\} .
$$

La constante implicite dans (9.15) dépend au plus de $\kappa, \varepsilon, J_{1}, J_{2}$.

Supposant cette majoration acquise, nous commençons par réduire l'étude au cas où $m$ impair et $T_{1}$ et $T_{2}$ satisfont $T_{j}(\boldsymbol{x}) \equiv m^{2}(\bmod 4)(j=1,2)$ : ces conditions impliquent $T_{j}(\boldsymbol{x}) \equiv 1(\bmod 4)$ pour tous les vecteurs $\boldsymbol{x}$ apparaissant dans la sommation.

En scindant la sommation selon les valeurs de $v_{2}((m, n))$ et $v_{2}\left(T_{j}(\boldsymbol{x})\right)$ et en faisant systématiquement usage de la relation $r(2 n)=r(n)$, nous obtenons

$$
\mathcal{Q}(\xi ; \boldsymbol{T}, \mathcal{R})=\sum_{j \geqslant 0} \mathcal{Q}_{1}\left(\xi / 2^{j} ; \boldsymbol{T}, \mathcal{R}\right)
$$

où l'on a posé

$$
\mathcal{Q}_{1}(\xi ; \boldsymbol{T}, \mathcal{R}):=\sum_{\substack{x \in \mathbb{Z}^{2} \cap \mathcal{R}(\xi) \\
2 \nmid(m, n)}} r\left(T_{1}(\boldsymbol{x})\right) r\left(T_{2}(\boldsymbol{x})\right)=\sum_{\substack {\left(k_{1}, k_{2}\right) \in \mathbb{N}^{2} \\
\begin{subarray}{c}{x \in \mathbb{Z}^{2} \cap \mathcal{R}(\xi) \\
2 \nmid(m, n) \\
v_{2}\left(T_{i}(\boldsymbol{x})\right)=k_{i}(i=1,2){ ( k _ { 1 } , k _ { 2 } ) \in \mathbb { N } ^ { 2 } \\
\begin{subarray} { c } { x \in \mathbb { Z } ^ { 2 } \cap \mathcal { R } ( \xi ) \\
2 \nmid ( m , n ) \\
v _ { 2 } ( T _ { i } ( \boldsymbol { x } ) ) = k _ { i } ( i = 1 , 2 ) } }\end{subarray}} r\left(T_{1}(\boldsymbol{x})\right) r\left(T_{2}(\boldsymbol{x})\right) .
$$

Notons, à fins de référence ultérieure, que les conditions arithmétiques de la somme intérieure s'écrivent encore

$$
2 \nmid(m, n), \quad T_{i}(\boldsymbol{x}) \equiv 2^{k_{i}}\left(\bmod 2^{k_{i}+2}\right) \quad(i=1,2) .
$$

Pour $\boldsymbol{k}=\left(k_{1}, k_{2}\right) \in \mathbb{N}^{2}$, désignons par $\mathcal{W}_{\boldsymbol{k}}$ l'ensemble des solutions dans $\{1\} \times$ $\mathbb{Z} / 2^{k_{1}+k_{2}+2} \mathbb{Z}$ du système

$$
T_{i}(\boldsymbol{x}) \equiv 2^{k_{i}}\left(\bmod 2^{k_{i}+2}\right) \quad(i=1,2),
$$

identifié avec une famille de représentants dans $\{1\} \times\left[0,2^{k_{1}+k_{2}+2}\right.$ [. L'ensemble des solutions $\boldsymbol{x}=(m, n)$ de $(9 \cdot 17)$ où $m$ est impair coïncide donc avec l'ensemble des vecteurs $\boldsymbol{x}$ satisfaisant à $\boldsymbol{x} \equiv \operatorname{m\alpha }\left(\bmod 2^{k_{1}+k_{2}+2}\right)$ lorsque $\alpha$ décrit $\mathcal{W}_{\boldsymbol{k}}$.

Symétriquement, désignons par $z_{k}$ l'ensemble des solutions dans $\mathbb{Z} / 2^{k_{1}+k_{2}+2} \mathbb{Z} \times\{1\}$ du système $T_{i}(X, 1) \equiv 2^{k_{i}}\left(\bmod 2^{k_{i}+2}\right)(i=1,2)$ telles que $X \equiv 0(\bmod 2)$, identifié avec une famille de représentants dans $\left[0,2^{k_{1}+k_{2}+2}\right.$ [. Alors l'ensemble des solutions $\boldsymbol{x}=(m, n)$ de $(9 \cdot 17)$ où $m$ est pair coïncide avec l'ensemble des vecteurs $\boldsymbol{x}$ vérifiant $\boldsymbol{x} \equiv n \boldsymbol{\alpha}\left(\bmod 2^{k_{1}+k_{2}+2}\right)$ où $n$ est impair et $\boldsymbol{\alpha}$ décrit $z_{k}$.

Pour chaque vecteur $\boldsymbol{k} \in \mathbb{N}^{2}$, nous introduisons, à l'image de (7·15), les matrices

$$
U_{\boldsymbol{\alpha}, \boldsymbol{k}}:= \begin{cases}\left(\begin{array}{cc}
1 & 0 \\
\beta & 2^{k_{1}+k_{2}+2}
\end{array}\right) & \text { si } \boldsymbol{\alpha}=(1, \beta) \in \mathcal{W}_{\boldsymbol{k}} \\
\left(\begin{array}{cc}
\beta & 2^{k_{1}+k_{2}+2} \\
1 & 0
\end{array}\right) & \text { si } \boldsymbol{\alpha}=(\beta, 1) \in \mathcal{Z}_{\boldsymbol{k}},\end{cases}
$$


et les formes binaires définies par

$$
T_{i, \boldsymbol{\alpha}, k}(\boldsymbol{x}):=T_{i}\left(U_{\boldsymbol{\alpha}, k} \boldsymbol{x}\right) / 2^{k_{i}} \quad\left(\boldsymbol{x} \in \mathbb{Z}^{2}, i=1,2\right) .
$$

Des manipulations identiques à celles qui conduisent à $(7 \cdot 21)$ permettent d'écrire

$$
\mathcal{Q}_{1}(\xi ; \boldsymbol{T}, \mathcal{R})=\sum_{\boldsymbol{k} \in \mathbb{N}^{2}} \sum_{\boldsymbol{\alpha} \in \mathcal{W}_{k} \cup z_{k}} \mathcal{Q}_{2}\left(\xi ; \boldsymbol{T}_{\boldsymbol{\alpha}, \boldsymbol{k}}, \mathcal{R}_{\boldsymbol{\alpha} k}\right),
$$

avec

$$
\mathcal{R}_{\boldsymbol{\alpha}, k}:=\left\{\boldsymbol{x} \in \mathbb{R}^{2}: U_{\boldsymbol{\alpha}, \boldsymbol{k}} \boldsymbol{x} \in \mathcal{R}\right\}, \quad \mathcal{Q}_{2}(\xi ; \boldsymbol{T}, \mathcal{R}):=\sum_{\substack{\boldsymbol{x} \in \mathbb{Z}^{2} \cap \mathcal{R}(\xi) \\ m \equiv 1 \bmod 2}} r\left(T_{1}(\boldsymbol{x})\right) r\left(T_{2}(\boldsymbol{x})\right)
$$

La deuxième étape consiste à appliquer (7·28) pour décomposer $r\left(T_{1, \boldsymbol{\alpha}, \boldsymbol{k}}(\boldsymbol{x})\right)$ avec les choix

$$
\left.\left.\left.\mathcal{J}_{1}:=[1, y], \quad \mathcal{J}_{2}:=\right] y, \vartheta^{2} \xi^{2} / y\right], \quad \mathcal{J}_{3}:=\right] \vartheta^{2} \xi^{2} / y, \infty\left[, \quad y=y_{1}:=\vartheta \xi /(\log \xi)^{6}\right.
$$

puis pour décomposer $r\left(T_{2, \boldsymbol{\alpha}, \boldsymbol{k}}(\boldsymbol{x})\right)$ avec à présent $y=y_{2}:=\vartheta \xi$ de sorte que $\mathfrak{J}_{2}=\varnothing$. L'analogue de la formule $(7 \cdot 29)$ s'écrit

$$
\mathcal{Q}_{1}(\xi ; \boldsymbol{T}, \mathcal{R})=\sum_{1 \leqslant j \leqslant 3} \sum_{h \in\{1,3\}} \mathcal{Q}_{1 j h}(\xi ; \boldsymbol{T}, \mathcal{R})
$$

avec pour $(j, h) \in\{1,2,3\} \times\{1,3\}$,

$$
\mathcal{Q}_{1 j h}(\xi ; \boldsymbol{T}, \mathcal{R})=2^{4} \sum_{\boldsymbol{k} \in \mathbb{N}^{2}} \sum_{\boldsymbol{\alpha} \in \mathcal{W}_{k} \cup z_{\mathbf{k}}} \sum_{\boldsymbol{x} \in \mathcal{R}_{\boldsymbol{\alpha}, k}(\xi) \cap \mathbb{Z}^{2}} r_{j}\left(T_{1, \boldsymbol{\alpha}, k}(\boldsymbol{x})\right) r_{h}\left(T_{2, \boldsymbol{\alpha}, \boldsymbol{k}}(\boldsymbol{x})\right)
$$

Lorsque $j \neq 2$, ces sommes sont estimées grâce au Lemme 9.3. Nous omettons les détails, qui sont standard.

Il reste à majorer

$$
\begin{aligned}
\mathcal{Q}_{12}(\xi ; \boldsymbol{T}, \mathcal{R}) & :=\sum_{h \in\{1,3\}} \mathcal{Q}_{12 h}(\xi ; \boldsymbol{T}, \mathcal{R}) \\
& =2^{4} \sum_{\substack{x \in \mathcal{R}(\xi) \cap \mathbb{Z}^{2} \\
2 \nmid(m, n)}} r\left(T_{2}(\boldsymbol{x})\right) \sum_{\substack{t_{1} \mid T_{1}(\boldsymbol{x}) \\
t_{1} \in \mathcal{I}_{2}}} \chi\left(t_{1}\right) .
\end{aligned}
$$

Nous procédons comme pour (7.42), mais les détails sont beaucoup plus simples car il n'est pas nécessaire ici d'introduire l'analogue des fonctions $\Upsilon$.

Dans l'idée d'appliquer l'inégalité de Cauchy-Schwarz, nous observons qu'en vertu du Théorème 4.2, du Lemme 4.3 et du Théorème 4.4, nous avons

$$
\begin{aligned}
\sum_{x \in \mathcal{R}(\xi) \cap \mathbb{Z}^{2}} r\left(T_{2}(\boldsymbol{x})\right) \Delta\left(T_{1}(\boldsymbol{x}), \chi\right)^{2} \ll\|\boldsymbol{T}\|^{\varepsilon} \sigma^{2} \xi^{2} E_{J_{1}, J_{2}}(\sigma \xi ; \Delta(\cdot, \chi), r) \\
\ll\|\boldsymbol{T}\|^{\varepsilon} \sigma^{2} \xi^{2} E_{J_{1}}(\sigma \xi ; \Delta(\cdot, \chi)) E_{J_{2}}(\sigma \xi ; r) \ll\|\boldsymbol{T}\|^{\varepsilon} \sigma^{2} \xi^{2} \mathcal{L}(\sigma \xi)^{\alpha}
\end{aligned}
$$

où $\alpha$ ne dépend que de $J_{1}$. Nous avons utilisé ici (3.12) sous la forme $E_{J_{2}}(\sigma \xi, r) \ll 1$. 
Posons alors

$$
\mathcal{B}_{1}(\xi):=\left\{\boldsymbol{x} \in \mathcal{R}(\xi) \cap \mathbb{Z}^{2}: \exists d \mid T_{1}(\boldsymbol{x}): \vartheta \xi /(\log \xi)^{6}<d \leqslant \vartheta \xi(\log \xi)^{6}\right\},
$$

et

$$
B_{1}(\xi):=\sum_{\boldsymbol{x} \in \mathcal{B}_{1}(\xi)} r\left(T_{2}(\boldsymbol{x})\right)
$$

Cette quantité est analogue à (7·39), le terme $r\left(T_{2}(\boldsymbol{x})\right)$ étant borné en moyenne. L'inégalité de Cauchy-Schwarz fournit

$$
\begin{aligned}
\mathcal{Q}_{12}(\xi ; \boldsymbol{T}, \mathcal{R})^{2} & \ll\left(\log _{2} \xi \sum_{\boldsymbol{x} \in \mathcal{B}_{1}(\xi)} r\left(T_{2}(\boldsymbol{x})\right) \Delta\left(T_{1}(\boldsymbol{x}), \chi\right)\right)^{2} \\
& \ll\left(\log _{2} \xi\right)^{2} B_{1}(\xi)\|\boldsymbol{T}\|^{\varepsilon} \sigma^{2} \xi^{2} \mathcal{L}(\sigma \xi)^{\alpha},
\end{aligned}
$$

Nous allons majorer $B_{1}(\xi)$ en faisant appel au Théorème 4.2. Soit $\left.\delta=\delta(\kappa) \in\right] 0,1[$ tel que $\|T\|^{\delta} \leqslant c_{0} \sigma \xi$, où $c_{0}$ est la constante apparaissant dans (4.6). Supposons alors, sans perte de généralité, que $\varepsilon \leqslant \delta / 4000$, fixons $z=z(\varepsilon)>3$ tel que $(\log 2) / \log z<\varepsilon$ et rappelons la définition $(7 \cdot 43)$ de $\Omega_{z}(n)$.

Soit $\eta \in] 0,1$. La contribution $B_{11}$ à $B_{1}(\xi)$ des vecteurs $\boldsymbol{x}$ tels que $\Omega_{z}\left(T_{1}(\boldsymbol{x})\right)>$ $(1+\eta) \log _{2} \xi$ n'excède pas

$$
\begin{aligned}
& \sum_{\boldsymbol{x} \in \mathcal{R}(\xi) \cap \mathbb{Z}^{2}} r\left(T_{2}(\boldsymbol{x})\right)(1+\eta)^{\Omega_{z}\left(T_{1}(\boldsymbol{x})\right)-(1+\eta) \log _{2} \xi} \\
& \ll \frac{\|\boldsymbol{T}\|^{\varepsilon} \sigma^{2} \xi^{2} E_{J_{1}}\left(2 \sigma \xi ; y^{\Omega_{z}}\right) E_{J_{2}}(2 \sigma \xi ; r)}{(\log \xi)^{(1+\eta) \log (1+\eta)}} \ll \frac{\|\boldsymbol{T}\|^{\varepsilon} \sigma^{2} \xi^{2}}{(\log \xi)^{Q(1+\eta)}} .
\end{aligned}
$$

Par ailleurs, en appliquant un analogue adéquat de $(5 \cdot 24)$, nous obtenons que la contribution $B_{12}$ à $B_{1}(\xi)$ des vecteurs $\boldsymbol{x}$ tels que $T_{1}(\boldsymbol{x}) \leqslant \vartheta^{2} \xi^{2} /(\log \xi)^{2}$ satisfait

$$
B_{12} \ll \frac{\|\boldsymbol{T}\|^{\varepsilon} \sigma^{2} \xi^{2}}{\sqrt{\log \xi}} .
$$

Tous les vecteurs $\boldsymbol{x}$ comptés dans la contribution complémentaire $B_{13}$ sont tels que $T_{1}(\boldsymbol{x})$ possède un diviseur $t$ tel que

$$
\Omega_{z}(t) \leqslant \frac{1}{2}(1+\eta) \log _{2} \xi, \quad \vartheta \xi /(\log \xi)^{8}<t \leqslant \vartheta \xi(\log \xi)^{6} .
$$

En effet, si $d$ désigne le diviseur de $T_{1}(\boldsymbol{x})$ apparaissant dans $(9 \cdot 23)$, ces conditions sont satisfaites pour $t=d$ ou $t:=T_{1}(\boldsymbol{x}) / d$. Il s'ensuit que

$$
B_{13} \leqslant \sum_{\substack{\vartheta \xi /(\log \xi)^{8}<t \leqslant \vartheta \xi(\log \xi)^{6} \\ \Omega_{z}(t) \leqslant \frac{1}{2}(1+\eta) \log _{2} \xi}} \sum_{\substack{x \in \mathcal{R}(\xi) \cap \mathbb{Z}^{2} \\ t \mid T_{1}(\boldsymbol{x})}} r\left(T_{2}(\boldsymbol{x})\right)
$$

L'analogue de (5·8) s'écrit

$$
\Lambda\left(t ; T_{1}\right)=\bigsqcup_{b \mid \psi_{1}(t)} b \Lambda^{*}\left(t_{b} ; T_{1}\right) \subset \bigcup_{b \mid \psi_{1}(t)} \bigcup_{\mathcal{A} \in \mathcal{U}_{T_{1}}\left(t_{b}\right)} b \mathcal{A}_{t_{b}}
$$

avec $t_{b}:=t /\left(t, b^{2}\right)$ et

$$
\psi_{1}(t):=\prod_{p^{\nu} \| t} p^{\lceil\nu / 2\rceil}
$$


Les ensembles $\mathcal{A}_{t_{b}}$ sont des réseaux de déterminant $t_{b}$ et $\left|\mathcal{U}_{T_{1}}\left(t_{b}\right)\right|=\varrho_{T_{1}}^{*}\left(t_{b}\right) / \varphi\left(t_{b}\right)$. De plus, nous avons

$$
\frac{\varrho_{T_{1}}^{+}(t)}{t^{2}}=\sum_{b \mid \psi_{1}(t)} \frac{\varrho_{T_{1}}^{*}\left(t_{b}\right)}{b^{2} \varphi\left(t_{b}\right) t_{b}} .
$$

Considérons l'un quelconque des réseaux $\mathcal{A}_{s}$ avec $s=t_{b}$, de base minimale $\left(\boldsymbol{v}_{s}, \boldsymbol{w}_{s}\right)$ choisie de sorte que $\left\|\boldsymbol{v}_{s}\right\| \leqslant\left\|\boldsymbol{w}_{s}\right\|$ et $\left\|\boldsymbol{v}_{s}\right\|\left\|\boldsymbol{w}_{s}\right\| \asymp s$. En raisonnant comme pour (5·13), nous obtenons

$$
\sum_{\boldsymbol{x} \in \mathcal{R}(\xi) \cap \mathcal{A}_{s}} r\left(T_{2}(\boldsymbol{x})\right) \ll \sum_{\substack{\lambda_{1} \leqslant 2 \sigma \xi / b\left\|\boldsymbol{v}_{s}\right\| \\ \lambda_{2} \leqslant 2 \sigma \xi / b\left\|\boldsymbol{w}_{s}\right\|}} r\left(T_{2}\left(\lambda_{1} \boldsymbol{v}_{s}+\lambda_{2} \boldsymbol{w}_{s}\right)\right) .
$$

Si $b\left\|\boldsymbol{w}_{s}\right\| \leqslant(\sigma \xi)^{1-\varepsilon}$, nous sommes en mesure d'appliquer le Théorème 4.2 avec $F_{1}=\mathbf{1}$, $F_{2}=r$, pour estimer la dernière somme. Nous obtenons ainsi

$$
\sum_{x \in \mathcal{R}(\xi) \cap \mathcal{A}_{s}} r\left(T_{2}(\boldsymbol{x})\right) \ll\|\boldsymbol{T}\|^{\varepsilon / 2} \frac{\sigma^{2} \xi^{2}}{b^{2} s} .
$$

La contribution globale de tels couples $(b, t)$ à la somme $B_{13}$ est donc

$$
\begin{aligned}
& \ll\|\boldsymbol{T}\|^{\varepsilon / 2} \sigma^{2} \xi^{2} \sum_{\substack{\vartheta \xi /(\log \xi)^{8}<t \leqslant \vartheta \xi(\log \xi)^{6} \\
\Omega_{z}(t) \leqslant \frac{1}{2}(1+\eta) \log _{2} \xi}} \sum_{b \mid \psi_{1}(t)} \frac{\varrho_{T_{1}}^{*}\left(t_{b}\right)}{b^{2} \varphi\left(t_{b}\right) t_{b}} \\
& \ll\|\boldsymbol{T}\|^{\varepsilon / 2} \sigma^{2} \xi^{2} \sum_{\substack{\vartheta \xi /(\log \xi)^{8}<t \leqslant \vartheta \xi(\log \xi)^{6} \\
\varrho_{T_{1}}^{+}(t)}}\left(\frac{1+\eta}{t^{2}}\right)^{\Omega_{z}(t)-\frac{1}{2}(1+\eta) \log _{2} \xi} \\
& \ll \frac{\|\boldsymbol{T}\|^{\varepsilon} \sigma^{2} \xi^{2}\left(\log _{2} \xi\right)}{(\log \xi)^{Q((1+\eta) / 2)}} .
\end{aligned}
$$

Si $b\left\|\boldsymbol{w}_{s}\right\|>(\sigma \xi)^{1-\varepsilon}$, alors

$$
\left\|\boldsymbol{v}_{s}\right\| \ll \frac{s}{\left\|\boldsymbol{w}_{s}\right\|} \ll \frac{\vartheta \xi(\log \xi)^{6}}{(\sigma \xi)^{1-\varepsilon}} \ll\|\boldsymbol{T}\|^{1 / 4}(\sigma \xi)^{\varepsilon}(\log \xi)^{6} \ll\|\boldsymbol{T}\|^{1 / 4}(\sigma \xi)^{2 \varepsilon} .
$$

La contribution globale de tels couples $(b, t)$ à la somme $B_{13}$ est donc

$$
\ll\|\boldsymbol{T}\|^{\varepsilon / 2}(\sigma \xi)^{1+\varepsilon} \sum_{\|\boldsymbol{v}\| \ll\|\boldsymbol{T}\|^{1 / 4}(\sigma \xi)^{2 \varepsilon}} \frac{F\left(T_{1}(\boldsymbol{v})\right)}{\|\boldsymbol{v}\|}
$$

où $F$ est la fonction multiplicative définie par

$$
F(n):=\sum_{t \mid n} \sum_{b \mid \psi_{1}(t)} \frac{\varrho_{T_{1}}^{*}\left(t_{b}\right)}{\varphi\left(t_{b}\right)} \quad(n \geqslant 1) .
$$

Un calcul standard permet de déduire des majorations du Lemme 3.2 que

$$
F(n) \ll n^{1 / 2+\varepsilon} \quad(n \geqslant 1) .
$$

Nous en déduisons que

$$
\begin{aligned}
\sum_{\|\boldsymbol{v}\| \ll\|\boldsymbol{T}\|^{1 / 4}(\sigma \xi)^{2 \varepsilon}} \frac{F\left(T_{1}(\boldsymbol{v})\right)}{\|\boldsymbol{v}\|} & \ll \sum_{\|\boldsymbol{v}\| \ll\|\boldsymbol{T}\|^{1 / 4}(\sigma \xi)^{2 \varepsilon}}\|\boldsymbol{T}\|^{1 / 2+\varepsilon}\|\boldsymbol{v}\|^{1+4 \varepsilon} \\
& \ll\|\boldsymbol{T}\|^{5 / 4+2 \varepsilon}(\sigma \xi)^{2 \varepsilon(3+2 \varepsilon)} \ll(\sigma \xi)^{7 \varepsilon}
\end{aligned}
$$


où la dernière estimation découle de $(9 \cdot 12)$ et où nous avons supposé sans perte de généralité que $\varepsilon \leqslant 1 / 5$.

En reportant dans $(9 \cdot 29)$ puis dans $(9 \cdot 30)$, nous obtenons

$$
B_{13} \ll \frac{\|\boldsymbol{T}\|^{\varepsilon} \sigma^{2} \xi^{2} \log _{2} \xi}{(\log \xi)^{Q((1+\eta) / 2)}} .
$$

En tenant compte de $(9 \cdot 25)$ et $(9 \cdot 26)$, nous obtenons finalement que la majoration suivante est valable pour tout $\eta \in] 0,1[$ :

$$
B_{1}(\xi) \ll \frac{\|\boldsymbol{T}\|^{\varepsilon}(\sigma+\vartheta)^{2} \xi^{2} \log _{2} \xi}{(\log \xi)^{\min \{Q(1+\eta), Q((1+\eta) / 2), 1 / 2\}}},
$$

d'où, en reportant dans $(9 \cdot 24)$,

$$
\mathcal{Q}_{12}(\xi ; \boldsymbol{T}, \mathcal{R}) \ll \frac{\|\boldsymbol{T}\|^{\varepsilon}(\sigma+\vartheta)^{2} \xi^{2}}{(\log \xi)^{\delta}}
$$

dès que $\delta<\max _{0<\eta<1} \min \left\{\frac{1}{4}, \frac{1}{2} Q\left(\frac{1}{2}(1+\eta)\right), \frac{1}{2} Q(1+\eta)\right\}$. Pour $\eta=0,359$, nous obtenons $\delta>0,0289>3 / 104$.

Cela achève la preuve du Lemme 9.1.

\subsection{Estimation asymptotique de $N_{P}(B)$ lorsque $P=P_{1} P_{2}$}

Nous allons établir ici la formule asymptotique

$$
N_{P}(B)=\left\{C_{P} \operatorname{vol}\left(\mathcal{R}_{P}\right)+O\left(\frac{1}{(\log B)^{1 / 70}}\right)\right\} B \log B \quad(B \rightarrow \infty),
$$

où $C_{P}$ est une constante indépendante de $H$ et $\mathcal{R}_{P}$ est défini par $(2 \cdot 1)$. La preuve repose sur les manipulations semblables à celles qui ont été effectuées au $\S 8$ pour établir (8.1).

Conformément à ce qui a été annoncé au $\S 9.2$, nous commençons par déduire du Lemme 9.1 une estimation générale pour la quantité $\mathcal{Q}_{\boldsymbol{T}}(\xi, \boldsymbol{d}, \boldsymbol{D} ; \mathcal{R})$ introduite en $(9 \cdot 5)$ lorsque $\boldsymbol{T}=\left(T_{1}, T_{2}\right)$ est un couple de formes quadratiques irréductibles sur $\mathbb{Q}[i]$, $\boldsymbol{D}=\left(D_{1}, D_{2}\right) \in \mathbb{N}^{* 2}$ et $\boldsymbol{d}=\left(d_{1}, d_{2}\right) \in \mathbb{N}^{* 2}$ avec $d_{j} \mid D_{j}(j=1,2)$.

Dans ce contexte, l'équivalent de $(5 \cdot 8)$ pour $\Lambda(\boldsymbol{d} ; \boldsymbol{T})$ s'écrit

$$
\Lambda(\boldsymbol{D} ; \boldsymbol{T})=\bigsqcup_{b \mid \psi_{1}\left(\left[D_{1}, D_{2}\right]\right)} b \Lambda^{*}\left(\boldsymbol{D}_{b} ; \boldsymbol{T}\right)
$$

où $\psi_{1}$ est la fonction définie en $(9 \cdot 27)$ et où l'on a posé

$$
\boldsymbol{D}_{b}=\left(D_{1, b}, D_{2, b}\right), \quad D_{1, b}:=\frac{D_{1}}{\left(D_{1}, b^{2}\right)}, \quad D_{2, b}:=\frac{D_{2}}{\left(D_{2}, b^{2}\right)} .
$$

Il s'agit maintenant de décrire les ensembles $\Lambda^{*}(\boldsymbol{s} ; \boldsymbol{T})\left(\boldsymbol{s}=\left(s_{1}, s_{2}\right) \in \mathbb{N}^{* 2}\right)$ en termes de réseaux. L'analyse est semblable à celle qui a été conduite au $\S 5.1$ pour $\Lambda^{*}(s ; T)$. Dans $\Lambda^{*}(\boldsymbol{s} ; \boldsymbol{T})$, nous définissons une relation d'équivalence en convenant que $\boldsymbol{x} \sim \boldsymbol{y}$ si, et seulement si, il existe $\lambda \in \mathbb{Z}$ tel que

$$
\boldsymbol{y} \equiv \lambda \boldsymbol{x}\left(\bmod s_{1} s_{2}\right),
$$

et nous notons que cela implique $\left(\lambda, s_{1} s_{2}\right)=1$. L'ensemble $\mathcal{U}_{\boldsymbol{T}}(\boldsymbol{s})$ des classes d'équivalence induit donc une partition de $\Lambda^{*}(\boldsymbol{s} ; \boldsymbol{T})$. Pour tout $\mathcal{A} \in \mathcal{U}_{\boldsymbol{T}}(s)$ et tout $\boldsymbol{x} \in \mathcal{A}$, nous avons

$$
\mathcal{A}=\left\{\boldsymbol{y} \in \Lambda^{*}(\boldsymbol{s} ; \boldsymbol{T}): \exists \lambda \in \mathbb{Z},\left(\lambda, s_{1} s_{2}\right)=1, \boldsymbol{y} \equiv \lambda \boldsymbol{x}\left(\bmod s_{1} s_{2}\right)\right\} .
$$


Comme $\lambda$ est déterminé modulo $s_{1} s_{2}$ de manière unique par $\boldsymbol{y}$, nous avons $\mid \mathcal{A} \cap\left[0, s_{1} s_{2}{ }^{2} \mid=\right.$ $\varphi\left(s_{1} s_{2}\right)$, d'où

$$
\left|\mathcal{U}_{\boldsymbol{T}}\left(s_{1}, s_{2}\right)\right|=\varrho_{\boldsymbol{T}}^{*}\left(s_{1} s_{2}\right) / \varphi\left(s_{1} s_{2}\right) .
$$

De plus, pour toute classe $\mathcal{A}$ de $\mathcal{U}_{\boldsymbol{T}}(\boldsymbol{s})$, tout diviseur $t$ de $s_{1} s_{2}$, et tout $\boldsymbol{x}$ de $\mathcal{A}$, l'ensemble

$$
\mathcal{A}_{t}:=\left\{\boldsymbol{y} \in \mathbb{Z}^{2}: \exists \lambda \in \mathbb{Z}, \boldsymbol{y} \equiv \lambda \boldsymbol{x}(\bmod t)\right\}
$$

est un sous-réseau de $\mathbb{Z}^{2}$ de rang 2 et de déterminant $t$, dont la définition ne dépend pas du choix de $\boldsymbol{x}$ dans $\mathcal{A}$.

Par analogie à $(2 \cdot 8)$, nous posons, pour $\ell \in \mathbb{N}^{*}, j=1,2$,

$$
d_{j, \ell}:=d_{j} /\left(d_{j}, \ell^{2}\right), \quad \ell_{1}:=\prod_{p^{\nu} \| \ell, p>2} p^{\nu}, \quad T_{j, \ell}(u, v):=\ell_{1}^{2} T_{j}(u, v) /\left(d_{j}, \ell^{2}\right) .
$$

Lorsque $\xi \geqslant 0, \mathcal{A} \in \mathcal{U}_{\boldsymbol{T}}(\boldsymbol{D}), b \in \mathbb{N}^{*}, e \mid d_{1, b} d_{2, b}$, nous posons

$$
\mathscr{G}_{d}(\xi, \boldsymbol{d}, \boldsymbol{D}, \mathcal{A} ; e, b):=\sum_{x \in e \mathcal{A}_{D_{1, b} D_{2, b} / e} \cap \mathcal{R}(\xi / b)} r\left(\frac{T_{1, b}(\boldsymbol{x})}{d_{1, b}}\right) r\left(\frac{T_{2, b}(\boldsymbol{x})}{d_{2, b}}\right) .
$$

Notant $\boldsymbol{T}_{b}:=\left(T_{1, b}, T_{2, b}\right)$, nous pouvons écrire

$$
\begin{aligned}
\mathcal{Q}_{\boldsymbol{T}}(\xi, \boldsymbol{d}, \boldsymbol{D} ; \mathcal{R}) & =\sum_{b \mid \psi_{1}\left(\left[D_{1}, D_{2}\right]\right)} \sum_{\boldsymbol{x} \in \Lambda^{*}\left(\boldsymbol{D}_{b} ; \boldsymbol{T}_{b}\right) \cap \mathcal{R}(\xi / b)} r\left(\frac{T_{1, b}(\boldsymbol{x})}{d_{1, b}}\right) r\left(\frac{T_{2, b}(\boldsymbol{x})}{d_{2, b}}\right) \\
& =\sum_{b \mid \psi_{1}\left(\left[D_{1}, D_{2}\right]\right)} \sum_{\mathcal{A} \in \mathcal{U}_{\boldsymbol{T}}\left(\boldsymbol{D}_{b}\right)} \sum_{e \mid D_{1, b} D_{2, b}} \mu(e) \mathscr{G}_{d}(\xi, \boldsymbol{d}, \boldsymbol{D}, \mathcal{A} ; e, b) .
\end{aligned}
$$

De la même manière que dans le cas $T$ irréductible, nous introduisons quelques notations afin d'appliquer le Lemme 9.1.

Pour chaque $b \mid \psi_{1}\left(\left[D_{1}, D_{2}\right]\right)$ et chaque réseau $\mathcal{A} \in \mathcal{U}_{\boldsymbol{T}}\left(\boldsymbol{D}_{b}\right)$, nous définissons une application linéaire $E$ telle que $E\left(\mathbb{Z}^{2}\right)=e \mathcal{A}_{D_{1, b} D_{2, b} / e}$. Posant $\mathcal{R}_{E}^{\star}:=\left\{\boldsymbol{x} \in \mathbb{R}^{2}: E \boldsymbol{x} \in \mathcal{R} / b\right\}$, nous avons donc

$$
\operatorname{vol}\left(\mathcal{R}_{E}^{\star}\right)=\frac{\operatorname{vol}(\mathcal{R})}{b^{2} \operatorname{dét} E}=\frac{\operatorname{vol}(\mathcal{R})}{b^{2} D_{1, b} D_{2, b} e} .
$$

Introduisant les formes binaires définies par $M_{j, E}(\boldsymbol{v})=T_{j, b}(E \boldsymbol{v}) / d_{j, b}\left(\boldsymbol{v} \in \mathbb{Z}^{2}, j=1,2\right)$, nous pouvons écrire, avec la notation $(9 \cdot 6)$,

$$
\mathscr{G}(\xi, \boldsymbol{d}, \boldsymbol{D}, \mathcal{A} ; e, b)=\sum_{\boldsymbol{v} \in \mathbb{Z}^{2} \cap \mathcal{R}_{E}^{\star}(\xi)} r\left(M_{1, E}(\boldsymbol{v})\right) r\left(M_{2, E}(\boldsymbol{v})\right)=\mathcal{Q}\left(\xi ; M_{1, E}, M_{2, E}, \mathcal{R}_{E}^{\star}\right) .
$$

Notant $\boldsymbol{M}_{E}:=\left(M_{1, E}, M_{2, E}\right)$, nous avons alors, avec la notation $(9 \cdot 7)$,

$$
\delta_{\boldsymbol{M}_{E}}\left(\mathcal{R}_{E}^{\star}\right)=\sup _{\boldsymbol{x} \in \mathcal{R}} \max _{j=1,2}\left\{\left|T_{j}(\boldsymbol{x})\right|^{1 / 2} / d_{j}\right\}:=\vartheta_{\boldsymbol{d}} \leqslant \delta_{\boldsymbol{T}}(\mathcal{R}) .
$$

Le Lemme 9.1 fournit

$$
\mathscr{G}(\xi, \boldsymbol{d}, \boldsymbol{D}, \mathcal{A} ; e, b)=\frac{\pi^{2} \mathcal{K}_{E} \operatorname{vol}(\mathcal{R}) \xi^{2}}{b^{2} D_{1, b} D_{2, b} e}+O\left(\left(\|\boldsymbol{T}\| D_{1} D_{2}\right)^{\varepsilon} \frac{\xi^{2}\left(\sigma_{\boldsymbol{T}} / b+\vartheta_{\boldsymbol{d}}\right)^{2}}{(\log \xi)^{3 / 104}}\right)
$$

où $\mathcal{K}_{E}:=\mathcal{K}\left(\boldsymbol{M}_{E}\right)=\prod_{p} \mathcal{K}_{p}\left(\boldsymbol{M}_{E}\right)$. 
Il reste à poser

$$
W(\boldsymbol{d}, \boldsymbol{D}, \boldsymbol{T}):=\sum_{b \mid \psi_{1}\left(\left[D_{1}, D_{2}\right]\right)} \sum_{\mathcal{A} \in \mathcal{U}_{\boldsymbol{T}}\left(D_{1, b}, D_{2, b}\right)} \sum_{e \mid D_{1, b} D_{2, b}} \frac{\mathcal{K}_{E} \mu(e)}{b^{2} D_{1, b} D_{2, b} e}
$$

(où la dépendance en $\boldsymbol{d}$ est implicite dans $\mathcal{K}_{E}$ ) et

$$
\begin{aligned}
n(\boldsymbol{D} ; \boldsymbol{T}) & :=\sum_{b \mid \psi_{1}\left(\left[D_{1}, D_{2}\right]\right)} \sum_{\mathcal{A} \in \mathcal{U}_{\boldsymbol{T}}\left(D_{1, b}, D_{2, b}\right)} \sum_{e \mid D_{1, b} D_{2, b}} \mu(e)^{2} \\
& =\sum_{b \mid \psi_{1}\left(\left[D_{1}, D_{2}\right]\right)} \frac{\varrho_{T_{1}, T_{2}}^{*}\left(D_{1, b}, D_{2, b}\right)}{\varphi\left(D_{1, b} D_{2, b}\right)} 2^{\omega\left(D_{1, b} D_{2, b}\right)} \ll_{\boldsymbol{T}}\left(D_{1} D_{2}\right)^{\varepsilon}
\end{aligned}
$$

pour obtenir

$$
\mathcal{Q}_{\boldsymbol{T}}(\xi, \boldsymbol{d}, \boldsymbol{D} ; \mathcal{R})=\pi^{2} \operatorname{vol}(\mathcal{R}) W(\boldsymbol{d}, \boldsymbol{D}, \boldsymbol{T}) \xi^{2}+O\left(n(\boldsymbol{D} ; \boldsymbol{T})\left(\|\boldsymbol{T}\| D_{1} D_{2}\right)^{\varepsilon} \frac{\xi^{2}\left(\sigma_{\boldsymbol{T}}+\vartheta\right)^{2}}{(\log \xi)^{3 / 104}}\right) .
$$

Parallèlement à (8.4), nous obtenons

$$
W(\boldsymbol{d}, \boldsymbol{D}, \boldsymbol{T})=\prod_{p} W_{p}(\boldsymbol{d}, \boldsymbol{D}, \boldsymbol{T})
$$

avec

$$
W_{2}(\boldsymbol{d}, \boldsymbol{D}, \boldsymbol{T}):=\lim _{\nu \rightarrow \infty} \frac{1}{2^{2 \nu-2}} \sum_{\substack{x \in\left(\mathbb{Z} / 2^{\nu} \mathbb{Z}\right)^{2} \\ T_{i}(\boldsymbol{x}) \in d_{i} \varepsilon_{\nu}(i=1,2)}} 1
$$

et

$$
\begin{aligned}
W_{p}(\boldsymbol{d}, \boldsymbol{D}, \boldsymbol{T}) & :=\lim _{\nu \rightarrow \infty} \frac{1}{p^{3 \nu+v_{p}\left(d_{1} d_{2}\right)}} \sum_{\substack{(\boldsymbol{x}, s, t) \in\left(\mathbb{Z} / p^{\nu} \mathbb{Z}\right)^{4} \\
T_{i}(\boldsymbol{x}) \equiv p^{v_{p}\left(d_{i}\right)}\left(s_{i}^{2}+t_{i}^{2}\right)\left(\bmod p^{\nu}\right) \\
T_{i}(\boldsymbol{x}) \equiv 0\left(\bmod p^{v_{p}\left(D_{i}\right)}\right)}} 1 \\
& =\left(1-\frac{\chi(p)}{p}\right)^{2} \sum_{\boldsymbol{\nu} \in \mathbb{N}^{2}} \chi\left(p^{\nu_{1}+\nu_{2}}\right) \frac{\varrho_{\boldsymbol{T}}\left(p^{N_{1}}, p^{N_{2}}\right)}{p^{2 N_{1}+2 N_{2}}}
\end{aligned}
$$

où l'on a posé dans la sommation

$$
N_{i}:=\max \left\{v_{p}\left(D_{i}\right), \nu_{i}+v_{p}\left(d_{i}\right)\right\} .
$$

Notant $\boldsymbol{d}_{\ell}:=\left(d_{1, \ell}, d_{2, \ell}\right)$, nous avons

$$
\mathcal{Q}_{\boldsymbol{T}}^{*}(\xi, \boldsymbol{d}, \boldsymbol{D} ; \mathcal{R})=\sum_{\ell \in \mathbb{N}^{*}} \mu(\ell) \mathcal{Q}\left(\xi / \ell, \boldsymbol{d}_{\ell}, \boldsymbol{D}_{\ell} ; \boldsymbol{T}_{\ell}, \mathcal{R}\right)
$$

de sorte que, pour $\mathcal{R}$ fixé, nous pouvons écrire

$$
\mathcal{Q}_{\boldsymbol{T}}^{*}(\xi, \boldsymbol{d}, \boldsymbol{D} ; \mathcal{R})=\pi^{2} \operatorname{vol}(\mathcal{R}) W^{*}(\boldsymbol{d}, \boldsymbol{D} ; \boldsymbol{T}) \xi^{2}+O\left(\frac{\left(d_{1} d_{2}\right)^{\varepsilon} \xi^{2}}{(\log \xi)^{3 / 104}}\right),
$$

avec

$$
W^{*}(\boldsymbol{d}, \boldsymbol{D} ; \boldsymbol{T})=\sum_{\ell \in \mathbb{N}^{*}} \frac{\mu(\ell)}{\ell^{2}} W\left(\boldsymbol{d}_{\ell}, \boldsymbol{D}_{\ell} ; \boldsymbol{T}_{\ell}\right)
$$


Parallèlement à $(8 \cdot 10)$, nous pouvons représenter $W^{*}(\boldsymbol{d}, \boldsymbol{D} ; \boldsymbol{T})$ sous forme d'un produit eulérien, soit

$$
W^{*}(\boldsymbol{d}, \boldsymbol{D} ; \boldsymbol{T})=\prod_{p} W_{p}^{*}(\boldsymbol{d}, \boldsymbol{D} ; \boldsymbol{T})
$$

$\operatorname{avec}^{(5)}$

$$
W_{2}^{*}(\boldsymbol{d}, \boldsymbol{D} ; \boldsymbol{T}):=\lim _{\nu \rightarrow \infty} \frac{1}{2^{2 \nu-2}} \sum_{\substack{\boldsymbol{x} \in\left(\mathbb{Z} / 2^{\nu} \mathbb{Z}\right)^{2} \\ T_{i}(\boldsymbol{x}) \in \mathcal{L}_{2} \mathcal{E}_{\nu}(i=1,2) \\ 2 \nmid\left(x_{1}, x_{2}\right)}} 1
$$

et

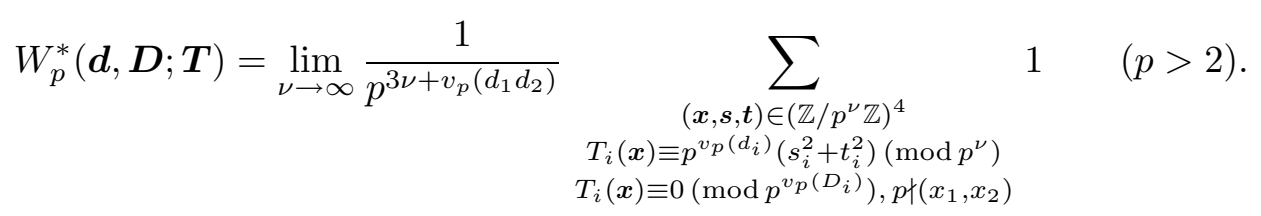

Conservant la notation $(9 \cdot 36)$, nous avons

$$
W_{p}^{*}(\boldsymbol{d}, \boldsymbol{D} ; \boldsymbol{T})=\left(1-\frac{\chi(p)}{p}\right)^{2} \sum_{\boldsymbol{\nu} \in \mathbb{N}^{2}} \chi\left(p^{\nu_{1}+\nu_{2}}\right) \frac{\varrho_{T}^{*}\left(p^{N_{1}}, p^{N_{2}}\right)}{p^{2 N_{1}+2 N_{2}}}\left(p>2, v_{p}\left(D_{1} D_{2}\right) \geqslant 1\right) .
$$

Lorsque $v_{p}\left(D_{1} D_{2}\right)=0$, cette formule reste valable quitte à y remplacer le terme correspondant à $\boldsymbol{\nu}=\mathbf{0}$ par $1-1 / p^{2}$.

Nous obtenons enfin en reportant dans (9.4), pour $\boldsymbol{t}=\left(t_{1}, t_{2}, t_{3}\right) \in \mathbb{N}^{* 3}$,

$$
S_{P_{1}, P_{2}}(\xi, \boldsymbol{t})=\pi^{2} \sum_{\varepsilon \in \mathcal{X}} \operatorname{vol}\left(\mathcal{R}_{P, \varepsilon}\right) W^{\dagger}(\boldsymbol{t}, \varepsilon) \xi^{2}+O\left(\frac{\left(t_{1} t_{2} t_{3}\right)^{\varepsilon} \xi^{2}}{(\log \xi)^{3 / 104}}\right),
$$

où nous avons posé, avec la notation $(9 \cdot 1)$,

$$
W^{\dagger}(\boldsymbol{t}, \varepsilon):=\sum_{k k^{\prime} \mid\left(\Delta, t_{1} t_{2}\right)} \frac{\mu\left(k^{\prime}\right)}{2^{\omega(k)}} W^{*}\left(\left(t_{1} t_{3}, t_{2} t_{3}\right),\left(\left[t_{1} t_{3}, k k^{\prime}\right],\left[t_{2} t_{3}, k k^{\prime}\right]\right) ; \varepsilon P_{1}, \varepsilon P_{2}\right) .
$$

Pour sommer sur $\boldsymbol{t}$, nous introduisons, comme en (8.15), un paramètre de troncature $D$. Le terme résiduel sera estimé à l'aide d'une majoration de $S_{P_{1}, P_{2}}(\xi, \boldsymbol{t})$ lorsque $t_{1} t_{2}>D$ - rappelons que dans ce contexte la variable $t_{3}$ demeure bornée, cf. $(9 \cdot 3)$.

Lemme 9.4. Soient $T_{1}, T_{2} \in \mathbb{Z}[X, Y]$ deux formes binaires de degré 2, irréductibles sur $\mathbb{Q}[i]$, non proportionnelles, et soit $\varepsilon>0$. Sous les conditions $\xi \geqslant 1, t_{1}, t_{2}, t_{3} \geqslant 1$ tels que $t_{3} \mid \operatorname{Res}\left(T_{1}, T_{2}\right), \mu^{2}\left(t_{3}\right) \mu^{2}\left(t_{1} t_{2}\right)=1$, nous avons

$$
S_{T_{1}, T_{2}}(\xi, \boldsymbol{t}) \ll\left(t_{1} t_{2}\right)^{\varepsilon}\left(\frac{\xi^{2}}{t_{1} t_{2}}+\xi^{1+\varepsilon}\right) .
$$

Nous omettons la démonstration qui est semblable à celle du Lemme 6.1.

De la même manière qu'en $(8 \cdot 15)$, nous pouvons montrer que, dans la somme $(9 \cdot 3)$, la contribution à $N_{P}(B)$ des triplets $\boldsymbol{t}$ tels que $t_{1} t_{2}>D$ est

$$
\ll \frac{B \log B}{D^{1-\varepsilon / 2}},
$$

5. Rappelons la définition des ensembles $\mathcal{E}_{\nu}$ en $(7 \cdot 3)$. 
pourvu que $D \leqslant \sqrt{\log B}$. Nous reportons alors l'estimation (9.39) pour $S_{P_{1}, P_{2}}(\xi, \boldsymbol{t})$ dans (9·3). La contribution du terme d'erreur est

$$
\ll B(\log B)^{1-3 / 104} D^{1+\varepsilon}
$$

Le choix $D=(\log B)^{3 / 208}$ fournit des termes d'erreur acceptables, l'exposant 1/70 apparaissant dans la formule (9.32) est $<3 / 208$.

Grâce au Lemme 2.2, nous obtenons que le terme principal vaut

$$
\begin{aligned}
& \frac{\pi^{2} B}{2^{5}} \sum_{\varepsilon \in \mathcal{X}} \operatorname{vol}\left(\mathcal{R}_{P, \varepsilon}\right) \sum_{d \leqslant D} \frac{\mu(d) \chi(d)}{d} \sum_{\substack{t \in \mathbb{N}^{* 3} \\
d=t_{1} t_{2}, t_{3} \mid \Delta}} \chi\left(t_{3}\right) \mu\left(t_{3}\right) W^{\dagger}(\boldsymbol{t}, \varepsilon) \sum_{n \leqslant \min \left\{B / d, B \sqrt{\|P\|} / d^{3 / 2}\right\}} \frac{f_{d}(n)}{n} \\
& =\frac{\pi B}{2^{5}} \sum_{\varepsilon \in \mathcal{X}} \operatorname{vol}\left(\mathcal{R}_{P, \varepsilon}\right) \sum_{d \leqslant D} \frac{\mu(d) r(d) \varphi^{\dagger}(d)}{d} \sum_{\substack{\boldsymbol{t} \in \mathbb{N}^{* 3} \\
d=t_{1} t_{2}, t_{3} \mid \Delta}} \chi\left(t_{3}\right) \mu\left(t_{3}\right) W^{\dagger}(\boldsymbol{t}, \varepsilon)\{\log B+O(\log 2 d)\} \\
& =\operatorname{vol}\left(\mathcal{R}_{P}\right) B \log B\left\{C_{P}+O\left(1 / D^{1-\varepsilon}\right)\right\}
\end{aligned}
$$

avec

$$
C_{P}:=\frac{\pi}{2^{5}} \sum_{\varepsilon \in X} \frac{\operatorname{vol}\left(\mathcal{R}_{P, \varepsilon}\right)}{\operatorname{vol}\left(\mathcal{R}_{P}\right)} \sum_{d \geqslant 1} \frac{\mu(d) r(d) \varphi^{\dagger}(d)}{d} \sum_{\substack{\boldsymbol{t} \in \mathbb{N}^{* 3} \\ d=t_{1} t_{2}, t_{3} \mid \Delta}} \chi\left(t_{3}\right) \mu\left(t_{3}\right) W^{\dagger}(\boldsymbol{t}, \varepsilon) .
$$

\subsection{Validation de la conjecture de Peyre}

Nous nous proposons ici d'établir la formule

$$
C_{P} \operatorname{vol}\left(\mathcal{R}_{P}\right)=C_{H}(V)
$$

en utilisant la méthode développée dans [5].

Il découle de la proposition 2 de [11] que l'ensemble des classes d'isomorphismes de torseurs versels au-dessus de $V$ ayant au moins un point rationnel est fini. Cela induit une partition canonique finie de l'ensemble des points rationnels de $V$, indexée par toute famille de représentants de ces classes d'isomorphismes. La constante de Peyre s'exprime alors naturellement comme somme des constantes asymptotiques de proportionnalité relatives aux diverses contributions des éléments de la partition — cf. la proposition 4.9 de [5] dans le cas d'un polynôme scindé.

Le résultant $\Delta$ étant défini par (9·1), nous posons

$$
\Delta_{1}:=\prod_{\substack{p \mid \Delta \\ p \equiv 1(\bmod 4)}} p, \quad \Delta_{3}:=\prod_{\substack{p \mid \Delta \\ p \equiv 3(\bmod 4)}} p
$$

Lorsque $y^{2}+z^{2}=t^{2} P_{1}(u, v) P_{2}(u, v)$ et $(u, v)=(y, z, t)=1$, il existe un unique signe $\varepsilon \in \mathcal{X}$ et un unique entier naturel $m_{3} \mid \Delta_{3}$ tels que

$$
\varepsilon P_{i}(u, v)>0, \quad 2 \mid v_{p}\left(\varepsilon P_{i}(u, v) / m_{3}\right) \quad(p \equiv 3(\bmod 4), i \in\{1,2\}) .
$$

On peut associer bijectivement à chaque couple $\left(\varepsilon, m_{3}\right)$ un torseur versel $\mathcal{T}_{\left(\varepsilon, m_{3}\right)}$ sur $V$ de telle manière que les $\mathcal{T}_{\left(\varepsilon, m_{3}\right)}\left(\varepsilon \in \mathcal{X}, m_{3} \mid \Delta_{3}\right)$ constituent dans leur ensemble un système complet de représentants des classes d'isomorphismes mentionnées plus haut. 
Les conditions $(9 \cdot 44)$ correspondent alors aux différents éléments de la partition induite, disons

$$
V(\mathbb{Q})=\bigsqcup_{\left(\varepsilon, m_{3}\right)} V_{\left(\varepsilon, m_{3}\right)}(\mathbb{Q}),
$$

de l'ensemble des points rationnels de $V$. Les calculs nécessaires à la vérification de ces assertions ayant été formalisés, dans le cas où $P$ est scindé, aux propositions 4.6 et 4.9 de [5], nous omettons les détails supplémentaires.

Supposons l'absence d'obstruction de Brauer-Manin pour la surface $V$. D'après un résultat établi dans [10], nous savons alors que, si $V$ a des points dans chaque complété de $\mathbb{Q}$, alors il existe un torseur $\mathcal{T}_{\left(\varepsilon, m_{3}\right)}$ tel qu'il en soit de même pour $\mathcal{T}_{\left(\varepsilon, m_{3}\right)}$. Or les torseurs $\mathcal{T}_{\left(\varepsilon, m_{3}\right)}(\mathbb{Q})$ vérifient le principe de Hasse. Cela établit, sous l'hypothèse précédente, l'existence d'un point rationnel de $\mathcal{T}_{\left(\varepsilon, m_{3}\right)}(\mathbb{Q})$ et donc de $V_{\left(\varepsilon, m_{3}\right)}(\mathbb{Q})$ et de $V$.

Le facteur de Batyrev-Tschinkel $\beta(V)$ apparaissant dans la conjecture de Peyre - voir [28], p. 335 - vaut ici ${ }^{(6)}$

$$
\beta(V)=|\operatorname{coker}(\operatorname{Br}(\mathbb{Q}) \rightarrow \operatorname{Br}(V))|=2 .
$$

Comme dans [5], ${ }^{(7)}$ nous écrivons $C_{H}(V) / \beta(V)$ comme somme des mesures de Tamagawa $^{(8)}$ des éléments de la partition $(9 \cdot 45)$, d'où

$$
C_{H}(V)=2 \sum_{\substack{\varepsilon \in \mathcal{X} \\ m_{3} \mid \Delta_{3}}} \boldsymbol{\omega}_{H}\left(V_{\left(\varepsilon, m_{3}\right)}(\mathbb{Q})\right) .
$$

Par définition de la mesure de Tamagawa, nous avons

$$
\boldsymbol{\omega}_{H}\left(V_{\left(\varepsilon, m_{3}\right)}(\mathbb{Q})\right)=\omega_{\infty}\left(\varepsilon, m_{3}\right) \prod_{p} \omega_{p}\left(\varepsilon, m_{3}\right) \quad\left(\varepsilon \in X, m_{3} \mid \Delta_{3}\right),
$$

où $\omega_{\infty}\left(\varepsilon, m_{3}\right)$ et $\omega_{p}\left(\varepsilon, m_{3}\right)$ sont les densités archimédienne et $p$-adique associées à la partie $V_{\left(\varepsilon, m_{3}\right)}(\mathbb{Q})$ de $V(\mathbb{Q})$. Cette formule illustre le fait que $V_{\left(\varepsilon, m_{3}\right)}(\mathbb{Q})$ vérifie le principe de Hasse.

Lorsque $p \nmid 2 \Delta_{3}$, nous avons

$$
\omega_{p}\left(\varepsilon, m_{3}\right)=\omega_{p}
$$

où $\omega_{p}$ est défini en $(8 \cdot 19)$ et calculé en $(8 \cdot 21)$ et $(8 \cdot 22)$.

Lorsque $p \mid 2 \Delta_{3}$, nous avons

$$
\omega_{p}\left(\varepsilon, m_{3}\right)=\lim _{\nu \rightarrow \infty} \frac{1}{p^{4 \nu}} \sum_{\substack{(u, v, x, y, t) \in\left(\mathbb{Z} / p^{\nu} \mathbb{Z}\right)^{5} \\ P(u, v) t^{2} \equiv x^{2}+y^{2}\left(\bmod p^{\nu}\right) \\ p \nmid(u, v), p \nmid(x, y, t) \\ 2 \mid v_{p}\left(P_{i}(u, v)\right)-\mu_{3}}} 1 .
$$

Les relations (9.44) impliquent que $P_{i}(u, v) / \varepsilon m_{3}$ s'écrit comme somme de deux carrés. La condition $P_{i}(u, v) \in \varepsilon m_{3} \varepsilon_{\nu}\left(\bmod 2^{\nu}\right)(i=1,2)$ est donc remplie pour tout $\nu \in \mathbb{N}$, et nous obtenons

$$
\omega_{2}\left(\varepsilon, m_{3}\right)=\lim _{\nu \rightarrow \infty} \frac{1}{2^{4 \nu}} \sum_{\substack{(u, v, x, y, t) \in\left(\mathbb{Z} / 2^{\nu} \mathbb{Z}\right)^{5} \\ P(u, v) t^{2}=x^{2}+y^{2}\left(\bmod 2^{\nu}\right) \\ 2 \nmid(u, v), 2 \nmid(x, y, t) \\ P_{i}(u, v) \in \varepsilon m_{3} \varepsilon_{\nu}}} 1=\lim _{\nu \rightarrow \infty} \frac{1}{2^{2 \nu}} \sum_{\substack{(u, v) \in\left(\mathbb{Z} / 2^{\nu} \mathbb{Z}\right)^{2} \\ P_{i}(u, v) \in \varepsilon m_{3} \varepsilon_{\nu}(i=1,2) \\ 2 \nmid(u, v)}} 1,
$$

où nous avons utilisé les conditions $2 \nmid t$ et $(8 \cdot 20)$.

6. Voir la proposition 5.1 de [10].

7. Voir p. 6 de ce travail.

8. Voir notamment la définition 4.6 de [28]. 
Enfin, comme dans le cas de $(8 \cdot 25)$, nous avons

$$
\omega_{\infty}\left(\varepsilon, m_{3}\right)=\frac{1}{2} \pi \operatorname{vol}\left(\mathcal{R}_{P, \varepsilon}\right) .
$$

Pour vérifier la relation (9.43), nous commençons par effectuer la factorisation $t_{3}=$ $m_{1} m_{3}$ avec $m_{1} \mid \Delta_{1}$ et $m_{3} \mid \Delta_{3}$. En reportant dans (9.42), nous obtenons alors une décomposition de la forme

$$
C_{P} \operatorname{vol}\left(\mathcal{R}_{P}\right)=\sum_{\substack{\varepsilon \in X \\ m_{3} \mid \Delta_{3}}} C_{P}\left(\varepsilon, m_{3}\right) \operatorname{vol}\left(\mathcal{R}_{P, \varepsilon}\right) .
$$

Le problème est donc réduit à la vérification des égalités

$$
C_{P}\left(\varepsilon, m_{3}\right) \operatorname{vol}\left(\mathcal{R}_{P, \varepsilon}\right)=2 \boldsymbol{\omega}_{H}\left(T\left(\varepsilon, m_{3}\right)\right) \quad\left(\varepsilon \in \mathcal{X}, m_{3} \mid \Delta_{3}\right) .
$$

Pour calculer $C_{P}\left(\varepsilon, m_{3}\right)$, nous introduisons ensuite les fonctions arithmétiques

$$
\begin{aligned}
V_{1}\left(t_{1}, t_{2}, m_{1}\right) & :=\sum_{k k^{\prime} \mid\left(\Delta, t_{1} t_{2}\right)} \frac{\mu\left(k^{\prime}\right)}{2^{\omega(k)}} \prod_{p \equiv 1(\bmod 4)} W_{p}^{*}\left(\left(t_{1} m_{1}, t_{2} m_{1}\right),\left(\left[t_{1} m_{1}, k k^{\prime}\right],\left[t_{2} m_{1}, k k^{\prime}\right]\right) ; P_{1}, P_{2}\right), \\
V_{2}\left(\varepsilon, m_{3}\right) & :=\frac{1}{4} W_{2}^{*}\left(\left(m_{3}, m_{3}\right),\left(m_{3}, m_{3}\right) ; \varepsilon P_{1}, \varepsilon P_{2}\right), \\
V_{3}\left(m_{3}\right) & :=\prod_{p \equiv 3(\bmod 4)} W_{p}^{*}\left(\left(m_{3}, m_{3}\right),\left(m_{3}, m_{3}\right) ; P_{1}, P_{2}\right),
\end{aligned}
$$

de sorte que, avec la notation (9.40), nous avons identiquement, pour $\boldsymbol{t}=\left(t_{1}, t_{2}\right)$,

$$
\frac{1}{4} W^{\dagger}(\boldsymbol{t}, \varepsilon)=V_{1}\left(t_{1}, t_{2}, m_{1}\right) V_{3}\left(m_{3}\right) V_{2}\left(\varepsilon, m_{3}\right) .
$$

(Nous avons exploité ici le fait que les facteurs $p$-adiques $W_{p}^{*}$ apparaissant dans $(9 \cdot 37)$ ne dépendent pas de $\varepsilon$ lorsque $p>2$.) Il suit

$$
C_{P}\left(\varepsilon, m_{3}\right)=\frac{1}{2} \pi V_{3}\left(m_{3}\right) V_{2}\left(\varepsilon, m_{3}\right) \sum_{d \geqslant 1} \frac{\mu(d) r(d) \varphi^{\dagger}(d)}{4 d} \sum_{\substack{t \in \mathbb{N}^{* 3} \\ d=t_{1} t_{2}, m_{1} \mid \Delta_{1}}} \mu\left(m_{1}\right) V_{1}\left(t_{1}, t_{2}, m_{1}\right)
$$

Notons que le facteur $\frac{1}{2}$ peut être interprété comme un produit eulérien. En effet, d'après $(2 \cdot 12)$, nous avons

$$
\prod_{p} \frac{1+\chi(p) / p}{1-\chi(p) / p}=\prod_{p>2} \frac{(1+\chi(p) / p)^{2}}{1-1 / p^{2}}=\frac{3}{4} \frac{\pi^{2}}{6}\left(\frac{2}{\pi}\right)^{2}=\frac{1}{2} .
$$

Posons encore

$$
c_{2}\left(\varepsilon, m_{3}\right):=V_{2}\left(\varepsilon, m_{3}\right)=\lim _{\nu \rightarrow \infty} \frac{1}{2^{2 \nu}} \sum_{\substack{(u, v) \in\left(\mathbb{Z} / 2^{\nu} \mathbb{Z}\right)^{2} \\ P_{i}(u, v) \in \varepsilon m_{3} \varepsilon_{\nu} \\ 2 \nmid(u, v)}} 1,
$$

et, lorsque $p$ est impair,

$$
\begin{gathered}
c_{p}\left(\varepsilon, m_{3}\right):=\frac{1+\chi(p) / p}{1-\chi(p) / p} \sum_{0 \leqslant \delta \leqslant 1}\left(-\frac{r(p)}{4(p+1)}\right)^{\delta} \sum_{\substack{\left(\delta_{1}, \delta_{2}\right) \in \mathbb{N}^{2} \\
\mu_{1} \leqslant \min \left\{1, v_{p}\left(\Delta_{1}\right)\right\} \\
\delta_{1}+\delta_{2}=\delta}}(-\chi(p))^{\mu_{1}} \\
\sum_{\substack{\kappa, \kappa^{\prime} \geqslant 0 \\
\kappa+\kappa^{\prime} \leqslant \min \left\{\delta, v_{p}\left(\Delta_{1}\right)\right\}}} \frac{(-1)^{\kappa^{\prime}}}{2^{\kappa}} W_{p}^{*}\left(\left(p^{n_{1}^{\prime}}, p^{n_{2}^{\prime}}\right),\left(p^{n_{1}}, p^{n_{2}}\right) ; P_{1}, P_{2}\right),
\end{gathered}
$$


avec

$$
n_{i}:=\max \left\{\kappa+\kappa^{\prime}, n_{i}^{\prime}\right\}, \quad n_{i}^{\prime}:=\delta_{i}+\mu_{1}+\mu_{3} \quad(i=1,2), \quad \mu_{j}:=v_{p}\left(m_{j}\right) \quad(j=1,3) .
$$

En exprimant la somme du membre de droite de (9.48) sous forme de produit eulérien et en utilisant $(9.49),(9.50)$ et $(9.51)$, nous obtenons

$$
C_{P}\left(\varepsilon, m_{3}\right)=\pi \prod_{p} c_{p}\left(\varepsilon, m_{3}\right)
$$

Grâce à $(9 \cdot 38)$, nous pouvons écrire

$$
\frac{c_{p}\left(\varepsilon, m_{3}\right)}{1-1 / p^{2}}=\sum_{0 \leqslant \delta \leqslant 1}\left(-\frac{r(p)}{4(p+1)}\right)^{\delta} \sum_{\substack{\left(\delta_{1}, \delta_{2}\right) \in \mathbb{N}^{2} \\ \mu_{1} \leqslant \min \left\{1, v_{p}\left(\Delta_{1}\right)\right\} \\ \delta_{1}+\delta_{2}=\delta}}(-\chi(p))^{\mu_{1}} f_{p}\left(\delta_{1}, \delta_{2}, \mu_{1}, \mu_{3}\right),
$$

où

$$
f_{p}\left(\delta_{1}, \delta_{2}, \mu_{1}, \mu_{3}\right):=\sum_{\boldsymbol{\nu} \in \mathbb{N}^{2}} \sum_{\substack{\kappa, \kappa^{\prime} \geqslant 0 \\ \kappa+\kappa^{\prime} \leqslant \min \left\{\delta, v_{p}\left(\Delta_{1}\right)\right\}}} \frac{(-1)^{\kappa^{\prime}}}{2^{\kappa}} \frac{\varrho_{P_{1}, P_{2}}^{*}\left(p^{N_{1}}, p^{N_{2}}\right)}{p^{2\left(N_{1}+N_{2}\right)}}
$$

avec

$$
N_{i}:=\max \left\{\kappa+\kappa^{\prime}, \nu_{i}+\delta_{i}+\mu_{1}+\mu_{3}\right\} \quad(i=1,2)
$$

Posons enfin

$$
\begin{aligned}
\varrho_{P_{1}, P_{2}}^{\dagger}\left(p^{\nu_{1}}, p^{\nu_{2}}\right) & :=\frac{1}{p^{2 \nu_{1}+2 \nu_{2}+2}}\left|\left\{\boldsymbol{x} \in \mathbb{Z} / p^{\nu_{1}+\nu_{2}+1} \mathbb{Z}: p^{\nu_{i}} \| P_{i}(\boldsymbol{x}), p \nmid\left(x_{1}, x_{2}\right)\right\}\right|, \\
\varrho_{P}^{\dagger}\left(p^{\nu}\right) & :=\frac{1}{p^{2 \nu+2}}\left|\left\{\boldsymbol{x} \in \mathbb{Z} / p^{\nu+1} \mathbb{Z}: p^{\nu} \| P(\boldsymbol{x}), p \nmid\left(x_{1}, x_{2}\right)\right\}\right| .
\end{aligned}
$$

Lorsque $p \equiv 1 \bmod 4$, et donc $\mu_{3}=0$, nous commençons par réécrire $f_{p}\left(\delta_{1}, \delta_{2}, \mu_{1}, 0\right)$ sous la forme

$$
f_{p}\left(\delta_{1}, \delta_{2}, \mu_{1}, 0\right)=\sum_{\substack{\kappa, \kappa^{\prime} \geqslant 0 \\ \kappa+\kappa^{\prime} \leqslant \min \left\{\delta, v_{p}\left(\Delta_{1}\right)\right\}}} \frac{(-1)^{\kappa^{\prime}}}{2^{\kappa}} \sum_{\substack{\boldsymbol{\nu} \in \mathbb{N}^{2} \\ \kappa^{\prime}+\kappa \leqslant N_{i}^{\prime}}}\left(\nu_{1}+1\right)\left(\nu_{2}+1\right) \varrho_{P_{1}, P_{2}}^{\dagger}\left(p^{N_{1}^{\prime}}, p^{N_{2}^{\prime}}\right)
$$

avec $N_{i}^{\prime}:=\nu_{i}+\delta_{i}+\mu_{1}$. Nous intervertissons les sommations et utilisons la formule

$$
\sum_{\substack{\kappa, \kappa^{\prime} \geqslant 0 \\ \leqslant \min \left\{\delta, N_{1}^{\prime}, N_{2}^{\prime}\right\}}} \frac{(-1)^{\kappa^{\prime}}}{2^{\kappa}}=\frac{1}{2^{\min \left\{\delta, N_{1}^{\prime}, N_{2}^{\prime}\right\}}} .
$$

Cela fournit

$$
f_{p}\left(\delta_{1}, \delta_{2}, \mu_{1}, 0\right)=\sum_{\boldsymbol{\nu} \in \mathbb{N}^{2}} \frac{\left(\nu_{1}+1\right)\left(\nu_{2}+1\right)}{2^{\min \left\{\delta, N_{1}^{\prime}, N_{2}^{\prime}\right\}}} \varrho_{P_{1}, P_{2}}^{\dagger}\left(p^{N_{1}^{\prime}}, p^{N_{2}^{\prime}}\right) .
$$

Posant alors $N_{i}^{\prime \prime}:=\nu_{i}+\delta_{i}$, nous obtenons

$$
\begin{aligned}
\sum_{m_{1} \leqslant \min \left\{1, v_{p}(\Delta)\right\}} & (-1)^{m_{1}} f_{p}\left(\delta_{1}, \delta_{2}, \mu_{1}, 0\right) \\
& =\sum_{\boldsymbol{\nu} \in \mathbb{N}^{2}}\left(\nu_{1}+1\right)\left(\nu_{2}+1\right)\left(\frac{\varrho_{P_{1}, P_{2}}^{\dagger}\left(p^{N_{1}^{\prime \prime}}, p^{N_{2}^{\prime \prime}}\right)}{2^{\min \left\{\delta, N_{1}^{\prime \prime}, N_{2}^{\prime \prime}\right\}}}-\frac{\varrho_{P_{1}, P_{2}}^{\dagger}\left(p^{N_{1}^{\prime \prime}+1}, p^{N_{2}^{\prime \prime}+1}\right)}{2^{\delta}}\right) \\
& =\sum_{\boldsymbol{\nu} \in \mathbb{N}^{2}}\left(\left(\nu_{1}+1\right)\left(\nu_{2}+1\right)-\nu_{1} \nu_{2}\right) \frac{\varrho_{P_{1}, P_{2}}^{\dagger}\left(p^{N_{1}^{\prime \prime}}, p^{N_{2}^{\prime \prime}}\right)}{2^{\min \left\{\delta, N_{1}^{\prime \prime}, N_{2}^{\prime \prime}\right\}}} \\
& =\sum_{\boldsymbol{\nu} \in \mathbb{N}^{2}}\left(\nu_{1}+\nu_{2}+1\right) \frac{\varrho_{P_{1}, P_{2}}^{\dagger}\left(p^{N_{1}^{\prime \prime}}, p^{N_{2}^{\prime \prime}}\right)}{2^{\min \left\{\delta, N_{1}^{\prime \prime}, N_{2}^{\prime \prime}\right\}}} .
\end{aligned}
$$


Lorsque les $N_{i}^{\prime \prime}$ sont fixés le nombre de couples $\left(\delta_{1}, \delta_{2}\right) \in\{(\delta, 0),(0, \delta)\}$ tel que $N_{i}^{\prime \prime}=\nu_{i}+\delta_{i}$ est égal à $2^{\min \left\{\delta, N_{1}^{\prime \prime}, N_{2}^{\prime \prime}\right\}}$. Il s'ensuit que

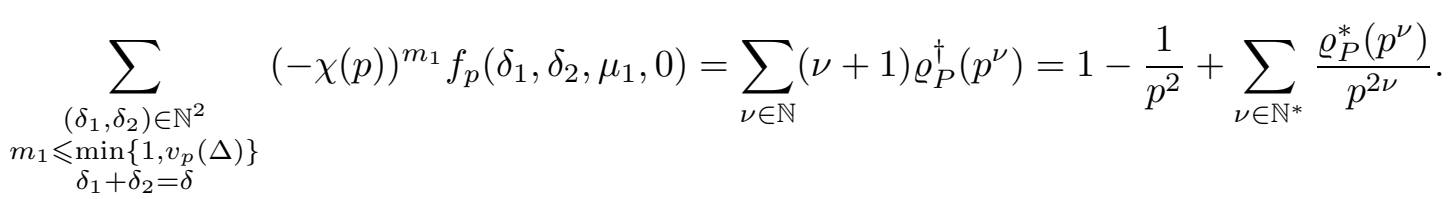

En reportant dans (9.52) et en effectuant une manipulation parallèle à celle $(8 \cdot 27)$, nous obtenons $c_{p}\left(m_{3}\right)=\omega_{p}$. Ainsi, dans le cas $p \equiv 1(\bmod 4)$, le facteur $c_{p}$ ne dépend pas de $m_{3}$.

Lorsque $p \equiv 3(\bmod 4)$, nous avons $r(p)=0$, d'où

$$
c_{p}\left(\varepsilon, m_{3}\right)=\left(1-\frac{1}{p^{2}}\right) \sum_{\boldsymbol{\nu} \in \mathbb{N}^{2}}(-1)^{\nu_{1}+\nu_{2}} \frac{\varrho_{P_{1}, P_{2}}^{*}\left(p^{\nu_{1}+\mu_{3}}, p^{\nu_{2}+\mu_{3}}\right)}{p^{2\left(\nu_{1}+\nu_{2}+2 \mu_{3}\right)}} .
$$

Il vient

$$
c_{p}\left(\varepsilon, m_{3}\right)=\left(1-\frac{1}{p^{2}}\right) \sum_{\boldsymbol{\nu} \in \mathbb{N}^{2}} \varrho_{P_{1}, P_{2}}^{\dagger}\left(p^{2 \nu_{1}+\mu_{3}}, p^{2 \nu_{2}+\mu_{3}}\right) .
$$

Or, pour chaque vecteur $\boldsymbol{x}$ compté dans $\varrho_{P}^{\dagger}\left(p^{2 \nu}\right)$ avec $\nu \geqslant 1$, il existe un unique couple d'entiers $\boldsymbol{\nu} \in \mathbb{N}^{2}$ tel que $p^{2 \nu_{i}+\mu_{3}} \| P_{i}(\boldsymbol{x})$. À $\mu_{3}$ fixé, nous pouvons donc sommer sur les exposants $\nu$ plutôt que sur les couples $\left(\nu_{1}, \nu_{2}\right)$.

Si $v_{p}\left(\Delta_{3}\right)=0$, nous avons $\mu_{3}=0$. Nous obtenons dans ce cas

$$
c_{p}\left(\varepsilon, m_{3}\right)=\left(1-\frac{1}{p^{2}}\right)\left\{\sum_{\nu \in \mathbb{N}} \varrho_{P}^{\dagger}\left(p^{2 \nu}\right)\right\}=\left(1-\frac{1}{p^{2}}\right)\left\{1-\frac{1}{p^{2}}+\sum_{\nu \in \mathbb{N}^{*}}(-1)^{\nu} \frac{\varrho_{P}^{*}\left(p^{\nu}\right)}{p^{\nu}}\right\}=\omega_{p} .
$$

Si $v_{p}\left(\Delta_{3}\right)=1$, nous avons $\mu_{3} \in\{0,1\}$. Nous observons alors que

$$
c_{p}(\varepsilon, 1)+c_{p}(\varepsilon, p)=\omega_{p} \quad\left(p \mid \Delta_{3}\right) .
$$

Grâce à (8·20), nous obtenons

$$
c_{p}\left(\varepsilon, m_{3}\right)=c_{p}\left(\varepsilon, p^{\mu_{3}}\right)=\lim _{\nu \rightarrow \infty} \frac{1}{p^{4 \nu}} \sum_{\substack{(u, v, x, y, t) \in\left(\mathbb{Z} / p^{\nu} \mathbb{Z}\right)^{5} \\ P(u, v)^{2} \equiv x^{2}+y^{2}\left(\bmod p^{\nu}\right) \\ p \nmid(u, v), p \nmid(x, y, t) \\ 2 \mid v_{p}\left(P_{i}(u, v)\right)-\mu_{3}}} 1=\omega\left(\varepsilon, m_{3}\right) .
$$

Il s'ensuit que

$$
C_{P}\left(\varepsilon, m_{3}\right) \operatorname{vol}\left(\mathcal{R}_{P, \varepsilon}\right)=2 \boldsymbol{\omega}_{H}\left(V_{\mathbb{Q}}\left(\varepsilon, m_{3}\right)\right),
$$

ce qui fournit la relation souhaitée $C_{P} \operatorname{vol}\left(\mathcal{R}_{P}\right)=C_{H}(V)$ en reportant dans $(9 \cdot 47)$ et en comparant avec $(9 \cdot 46)$.

\section{Bibliographie}

[1] R. de la Bretèche \& T.D. Browning, Sums of arithmetic functions over values of binary forms, Acta Arith., $125 \mathrm{n}^{\circ} 3$ (2006), 291-304.

[2] R. de la Bretèche \& T.D. Browning, Binary linear forms as sums of two squares, Compositio Mathematicae, 144 (6), (2008), 1375-1402.

[3] R. de la Bretèche \& T.D. Browning, Le problème des diviseurs pour des formes binaires de degré 4 , J. reine angew. Math., 646, (2010), 1-44. 
[4] R. de la Bretèche \& T.D. Browning, Binary forms of two squares and Châtelet surfaces, Israel Journal of Math., 191 (2012), 973-1012.

[5] R. de la Bretèche, T.D. Browning \& E. Peyre, On Manin's conjecture for a family of Châtelet surfaces, Annals of Math., 175 (2012), 1-47.

[6] R. de la Bretèche \& G. Tenenbaum, Oscillations localisées sur les diviseurs, Journal of London Math. Soc., (2012) 85 (3), 669-693.

[7] R. de la Bretèche \& G. Tenenbaum, Moyennes de fonctions arithmétiques de formes binaires, Mathematika, 58 (2012), 290-304.

[8] T.D. Browning, Quantitative arithmetic of projective varieties Progress in Math. 277, Birkhäuser, 2009.

[9] T.D. Browning, Linear growth for Châtelet surfaces, Math. Annalen 346 (2010), 41-50.

[10] J.-L. Colliot-Thélène, D. Coray \& J.-J. Sansuc, Descente et principe de Hasse pour certaines variétés rationnelles, J. reine angew. Math. 320 (1980), 150-191.

[11] J.-L. Colliot-Thélène \& J.-J. Sansuc, La descente sur les variétés rationnelles, Journées de géométrie algébrique d'Angers (1979) (A. Beauville, éd.), Sijthoff \& Noordhoff, Alphen aan den Rijn, 1980, pp. 223-237.

[12] J.-L. Colliot-Thélène, J.-J. Sansuc \& P. Swinnerton-Dyer, Intersections of two quadrics and Châtelet surfaces. I. J. reine angew. Math. 373 (1987), 37-107.

[13] J.-L. Colliot-Thélène, J.-J. Sansuc \& P. Swinnerton-Dyer, Intersections of two quadrics and Châtelet surfaces. II. J. reine angew. Math. 374 (1987), 72-168.

[14] R.J. Cook, Simultaneous quadratic equations, J. London Math. Soc. (2) 4 (1971), 319-326.

[15] S. Daniel, On the divisor-sum problem for binary forms, J. reine angew. Math. 507 (1999), 107-129.

[16] U. Derenthal, On a constant arising in Manin's conjecture for del Pezzo surfaces, Math. Res. Lett. 14 (2007), $\mathrm{n}^{\circ} 3,481-489$.

[17] R.R. Hall and G. Tenenbaum, Divisors, Cambridge tracts in mathematics 90, Cambridge University Press (1988, paperback ed. 2008).

[18] D.R. Heath-Brown, Diophantine approximation with square-free numbers, Math. Zeit. 187 (1984), $335-344$.

[19] D. R. Heath-Brown, Linear relations amongst sums of two squares. Number theory and algebraic geometry, 133-176, London Math. Soc. Lecture Note Ser. 303 CUP, 2003.

[20] H. Heilbronn, Zeta-functions and L-functions, Algebraic Number Theory (Proc. Instructional Conf., Brighton, 1965), 204-230 (1967), Thompson, Washington, D.C.

[21] K. Henriot, Nair-Tenenbaum bounds uniform with respect to the discriminant, Math. Proc. Camb. Phil. Soc., à paraître.

[22] C. Hooley, A new technique and its applications to the theory of numbers, Proc. London Math. Soc. (3) 38 (1979), 115-151.

[23] V.A. Iskovskih, A counterexample to the Hasse principle for systems of two quadratic forms in five variables, Mat. Zametki 10 (1971), 253-257; English transl. in Math. Notes 10 (1971), 575-577.

[24] H. Maier \& G. Tenenbaum, On the normal concentration of divisors, 2, Math. Proc. Camb. Phil. Soc. $147 \mathrm{n}^{\mathrm{o}} 3$ (2009), 593-614.

[25] G. Marasingha, On the representation of almost primes by pairs of quadratic forms, Acta Arith., 124 (2006), no. 4, 327-355.

[26] T. Nagell, Introduction to number theory. 2nd ed., Chelsea, 1964.

[27] E. Peyre, Hauteurs et mesures de Tamagawa sur les variétés de Fano, Duke Math. J. 79 (1995), 101-218.

[28] E. Peyre, Points de hauteurs bornée, topologie adélique et mesures de Tamagawa, J. théor. nombres Bordeaux 15 (2003) $\mathrm{n}^{\circ} 1,319-349$.

[29] A. Skorobogatov, Torsors and rational points, Cambridge Tracts in Mathematics, 144, Cambridge University Press, Cambridge, 2001, viii+187 pp.

[30] C.L. Stewart, On the number of solutions of polynomial congruences and Thue equations. J. Amer. Math. Soc. 4 (1991), 793-835.

[31] G. Tenenbaum, Sur une question d'Erdős et Schinzel, II, Inventiones Math. 99 (1990), 215-224.

[32] G. Tenenbaum, Introduction à la théorie analytique et probabiliste des nombres, troisième édition, coll. Échelles, Belin, 2008, 592 pp.

\author{
Régis de la Bretèche \\ Institut de Mathématiques de Jussieu \\ UMR 7586 \\ Université Paris Diderot-Paris 7 \\ UPR de Mathématiques, case 7012 \\ Bâtiment Chevaleret \\ 75205 Paris Cedex 13 \\ France \\ breteche@math.jussieu.fr
}

\author{
Gérald Tenenbaum \\ Institut Élie Cartan \\ Université de Lorraine \\ BP 70239 \\ 54506 Vandœuvre-lès-Nancy Cedex \\ France \\ gerald.tenenbaum@univ-lorraine.fr
}

